\title{
Determinant Bundles and Virasoro Algebras
}

\author{
A. A. Beilinson ${ }^{1}$ and V. V. Schechtman ${ }^{2}$ \\ 1 125319, Chernyakhovsky street, 5, apt. 144, Moscow, USSR \\ ${ }^{2}$ Institute of Problems of Microelectronics Technology and Superpure Materials, \\ SU-142432 Chernogolovka, Moscow district, USSR
}

To the memory of Vadik Knizhnik (20.2.1962-25.12.1987)

\begin{abstract}
We consider the interplay of infinite-dimensional Lie algebras of Virasoro type and moduli spaces of curves, suggested by string theory. We will see that the infinitesimal geometry of determinant bundles is governed by Virasoro symmetries. The Mumford forms are just invariants of these symmetries. The representations of Virasoro algebra define (twisted) $\mathscr{D}$-modules on moduli spaces; these $\mathscr{D}$-modules are equations on correlators in conformal field theory.
\end{abstract}

\section{Introduction}

0.1 . Let $X$ be a smooth (complex, or algebraic) variety, and $E$ a vector bundle on $X$. The Atiyah (or current) algebra $\mathscr{A}_{E}$ of $E$ is the algebra of infinitesimal symmetries of $E$ which is the extension of the tangent sheaf $\mathscr{T}_{X}$ by End $E$. Now let $\pi: X \rightarrow S$ be a proper smooth map with 1-dimensional fibers (so $X$ is a family of curves); then, according to Grothendieck, we have a determinant line bundle $\lambda_{E}=\operatorname{det} R \pi_{*} E$ with fibers

$$
\lambda_{E, s}=\operatorname{det} H^{0}\left(X_{s}, E_{s}\right) \otimes \operatorname{det} H^{1}\left(X_{s}, E_{s}\right)^{-1} .
$$

First problem: find $\mathscr{A}_{\lambda_{E}}$. It appears that this may be done in a very simple way, using $\mathscr{A}_{E}$ only. Namely, we construct (purely locally) a canonical differential graded Lie superalgebra ${ }^{\operatorname{tr}} \mathscr{A}_{E}$ on $X$ such that $H^{i}\left({ }^{\mathrm{tr}} \mathscr{A}_{E}\right)=0$ for $i \neq 0,-2, H^{0}\left({ }^{\mathrm{tr}} \mathscr{A}_{E}\right)$ $=\pi^{-1} \mathscr{T}_{S}, \quad H^{-2}\left({ }^{\operatorname{tr}} \mathscr{A}_{E}^{*}\right)=\mathbb{C} \quad$ (in the classical topology of $X$ ). Then $\pi\left({ }^{\operatorname{tr}} \mathscr{A}_{E}^{*}\right):=R^{0} \pi_{*}{ }^{\operatorname{tr}} \mathscr{A}_{E}^{*}$ is the extension of $\mathscr{T}_{S}$ by $\mathcal{O}_{S}$-and it is canonically isomorphic to $\mathscr{A}_{\lambda_{E}}$. This subject is treated in Sect. 2. In fact, $\pi\left({ }^{\mathrm{tr}} \mathscr{A}_{E}^{*}\right)$ is determined by a certain quotient of ${ }^{\text {tr }} \mathscr{A}_{E}$ : this is how the Virasoro algebra, the canonical central extension of $\mathscr{A}_{E}$, arises.

0.2. In Sect. 3 we show that this construction gives the differential equations for Mumford discriminant forms. More precisely, the natural action of vector fields (Lie derivative) on the sheaf $\omega^{j}$ of relative $j$-differentials on $X$ extends to the isomorphism between certain canonical subalgebras of $\mathscr{A}_{\omega}$ and $\mathscr{A}_{\mathcal{O}_{x}}$. This 
isomorphism has "central charge" $c_{j}=6 j^{2}-6 j+1$, thus it defines the canonical integrable connection on $\lambda_{j} / \lambda_{0}^{c_{j}}$ (here $\lambda_{j}=\lambda_{\omega^{\prime}}$ ); the Mumford forms $\mu_{j}$ are just the horizontal sections. This local definition of $\mu_{j}$ as opposed to the usual global one is quite convenient for calculations.

0.3. Section 4 is devoted to the Virasoro action on moduli. Consider the moduli space $\hat{\mathscr{M}}$ of triples $(X, x, t)$, where $X$ is a (compact smooth) curve, $x \in X$ a point, and $t$ a (formal) parameter at $x$. A version of Kodaira-Spencer construction shows that the Lie algebra $\mathscr{L}=\mathbb{C}((t)) \partial_{t}$ of formal vector fields acts naturally on $\mathscr{M}$, so that $\mathscr{L}_{+}=\mathbb{C}[[t]] t \partial_{t}$ changes the parameter $t$ leaving $(X, x)$ fixed, $\partial_{t}$ moves $x$, and the fields with poles may change the complex structure on $X$. In fact, this action is transitive (i.e. the Lie algebra is mapped surjectively onto the tangent space to any triple) with the stabilizer of $(X, x, t)$ being equal to the image of the expansion at $x$ map $\widetilde{T}_{X}(X \backslash\{x\}) \rightarrow \mathscr{L}$. This "Virasoro uniformisation" complements in some strange way the Teichmüller one.

A variant: we may consider $n$ different points $x_{1}, \ldots, x_{n}$ with parameters $t_{1}, \ldots, t_{n}$ at them to get the action of $\mathscr{L}^{n}$ on the corresponding moduli spaces. We may also add to our considerations a bundle (together with trivialisations at $x_{i}$ ). Now the result of Sect. 2 implies that this $\mathscr{L}$-action on the moduli space lifts canonically to the $\hat{\mathscr{L}}$-action on the determinant bundle with central charge 1 .

This construction was also (simultaneously) found by Kontsevich [15]; the case of deformation of a vector bundle (with the curve fixed) was considered extensively in the soliton theory [19]. Note that the central charge of Virasoro action on $\lambda_{j} / \lambda_{0}^{c_{j}}$ vanishes (cancellation of anomalies), and $\mu_{j}$ are just the Virasoro invariant sections (hence the Polyakov measure is completely determined by its invariance properties: first by invariance with respect to the conformal group, and then by Virasoro invariance). See also [25-28] for closely related subjects.

More generally, any $\hat{\mathscr{L}}$-module defines a (twisted) $\mathscr{D}$-module on $\hat{\mathscr{M}}$; this way one gets the differential equations for correlators [6] from the equations for singular vectors in a Verma module (this was explained to us by V. Drinfeld). In the degenerate case these $\mathscr{D}$-modules are, as Drinfeld has shown, just the bundles with (twisted) integrable connections; it would be very interesting to find the corresponding (projective) monodromy representations of the Teichmüller group explicitly. Compare with ideas of Manin [16] that were a starting point for our paper.

0.5. In Sect. 5 we describe how a pair of $\mathrm{C}^{\infty}$-connections, one on $E$, another on $\Omega_{X / S}^{1}$, determine a connection on the determinant bundle with the curvature calculated by the Riemann-Roch-Grothendieck formula.

0.6. In Sect. 6 we describe the algebras ${ }^{\text {tr }} \mathscr{A}_{E}$ for a bundle equipped with a connection having logarithmic singularities. In fact, we prove a more general assertion that describes the functoriality of these algebras with respect to morphisms of Atiyah algebras "with logarithmic singularities." As a consequence we will see how the determinant sheaves $\lambda_{j}$ behave with respect to ramified coverings.

0.7. Finally, in the Appendix we prove a local Riemann-Roch theorem for Atiyah algebras inspired by Deligne's paper [11]. This result generalizes the "cancellation 
of anomalies" isomorphism of Sect. 3. It seems that, by analogy with Sect. 6, the appropriate generalization of Sect. 5 should describe the functoriality of the RR-isomorphism A 3.3 with respect to $\mathbb{C}^{\infty}$-morphisms of Atiyah algebras.

Note in conclusion that it would be very natural to "integrate" the results of the present paper and construct some objects of Lie group type on $X$ that after integrating along the fibers should give determinant bundles themselves and not only their algebras of infinitesimal symmetries. The corresponding local RiemannRoch isomorphism should give after integrating the Deligne isomorphism [11], cf. $2.7,6.3 .7$.

\section{1. $\Omega$-Extensions of Atiyah Algebras}

\subsection{Atiyah Algebras - a Dictionary}

From now on $X$ will be a smooth variety, $\mathscr{T}_{X}$ the tangent bundle.

1.1.1. Let $E$ be a vector bundle on $X$. Put $\mathscr{D}_{E}=\operatorname{Diff}(E, E)=U \mathscr{D}_{i}, \mathscr{D}_{i}=\mathscr{D}_{E i}$ is the set of differential operators of degree $\leqq i$; we have $\mathscr{D}_{i} / \mathscr{D}_{i-1}=\operatorname{End} E \otimes S^{i}\left(\mathscr{T}_{X}\right)$. Define the Atiyah algebra of $E$ setting $\mathscr{A}_{E}=\left\{\partial \in \mathscr{D}_{1}: \varepsilon(\partial):=\operatorname{symb}_{1}(\partial) \in \mathscr{T}_{X}=\operatorname{id}_{E} \otimes \mathscr{T}_{X}\right.$ $C$ End $\left.E \otimes \mathscr{T}_{X}\right\} ;$ we have $\mathscr{D}_{o}=$ End $E \subset \mathscr{A}_{E} \subset \mathscr{D}_{1}, \mathscr{A}_{E} /$ End $E \stackrel{\varepsilon}{\rightarrow} \mathscr{T}_{X}$. Clearly $\mathscr{A}_{E}$ is just the Lie algebra of infinitesimal symmetries of $(X, E)$ : its elements are pairs $(\tau, \tilde{\tau})$, where $\tau \in \mathscr{T}_{X}$ is a vector field, and $\tilde{\tau}$ is the action of $\tau$ on $E$ [we have $\left.(\tau, \tilde{\tau})=(\varepsilon(\partial), \partial)\right]$.

This basic example motivates the following general

\subsubsection{Definition. An Atiyah algebra on $X$ consists of}

- a sheaf of associative $\mathcal{O}_{X}$-algebras $R$;

- a Lie algebra extension $O \rightarrow R^{\mathrm{Lie}} \rightarrow \mathscr{A} \stackrel{\varepsilon}{\rightarrow} \mathscr{T}_{X} \rightarrow O$ (here $R^{\mathrm{Lie}}$ is $R$ endowed with the bracket $[a, b]=a b-b a$, and $\mathscr{T}_{X}$ with the usual Lie bracket);

- a left $\mathscr{O}_{X}$-module structure on $\mathscr{A}$ compatible with the $\mathcal{O}_{X}$-module structures on $R$ and $\mathscr{T}_{X}$.

For these structures the following identities should hold: $[\alpha, a b]=[\alpha, a] b$ $+a[\alpha, b]$ for any $\alpha \in \mathscr{A}$ and either $a, b \in R$ or $a \in \mathcal{O}_{X}, b \in \mathscr{A} ;[\alpha, f]=\varepsilon(\alpha)(f)$ for $f \in \mathcal{O}_{X} \subset R$.

We will call Atiyah algebras with given $R$ simply $R$-Atiyah algebras; hence $\mathscr{A}_{E}$ is EndE-Atiyah algebra. Note that an Atiyah algebra has also natural right $\mathcal{O}_{X}$-module structure given by the formula $\alpha f:=f \alpha+\varepsilon(\alpha)(f)$. The Atiyah algebras form a category in an obvious way (so we have a forgetting functor $(R, \mathscr{A}) \sim \rightarrow R$ ). 1.1.3. The following definition and lemma, though not of much use in what follows, help one to feel at ease with 1.1.2.

Definition. An algebra of differential operators, do-algebra for short, is a sheaf of associative rings $\mathscr{D}$ on $X$ together with an increasing filtration $\mathscr{D}_{0} \subset \mathscr{D}_{l} \subset \ldots, \mathscr{D}$ $=\cup \mathscr{D}_{i}$, and a ring morphism $\mathcal{O}_{X} \stackrel{i}{\rightarrow} \mathscr{D}_{0}$ such that:

(i) $i$ maps $\mathcal{O}_{X}$ into the center of $\mathscr{D}_{o}$ (i.e. $\mathscr{D}_{o}$ is $\mathcal{O}_{X}$-algebra).

(ii) For $a \in \mathscr{D}_{l}$ and $f \in \mathscr{O}_{X}$ one has $\varepsilon(a)(f):=[a, i(f)] \in \mathscr{D}_{o}$, and this map $\varepsilon: \mathscr{D}_{l} / \mathscr{D}_{o} \rightarrow \operatorname{Der}\left(\mathcal{O}_{X}, \mathscr{D}_{o}\right)=\mathscr{T}_{X} \otimes \mathscr{D}_{o}$ is an isomorphism.

(iii) $\varepsilon$ defines an isomorphism $\mathrm{Gr}: \mathscr{D} \simeq \mathrm{S}\left(\mathscr{T}_{X}\right) \otimes \mathscr{D}_{0}$.

For an $R$-Atiyah algebra $\mathscr{A}$ let $\mathscr{D} \mathscr{A}$ be the quotient of the $\mathrm{I}(\mathscr{A})$, the universal envelopping algebra of $\mathscr{A}$, by the relations $a \cdot b=a b$, where either $a, b \in R$, or $a \in \mathcal{O}_{X}$, 
$b \in \mathscr{A}$ [here the left-hand side is the product in $\mathrm{U}(\mathscr{A})$, the right-hand side is either the product in $R$, or comes from the $\mathcal{O}_{X}$-module structure on $\left.\mathscr{A}\right]$, and $l_{\mathrm{U}(\mathscr{A})}=l_{R}$. Clearly $\mathscr{D}_{\mathscr{A}}$ is a do-algebra with $\mathscr{D}_{o}=R, \mathscr{D}_{l}=\mathscr{A} \cdot R, \mathscr{D}_{i}=\mathscr{A} \cdot \mathscr{D}_{i-l}$. Conversely, if $\mathscr{D}$ is a do-algebra, then $\mathscr{A}_{\mathscr{D}}=\mathscr{T}_{X} \underset{\mathscr{D} / \mathscr{D}_{0}}{\times} \mathscr{D}_{l}$ [the morphism $\mathscr{T}_{X} \rightarrow \mathscr{D}_{l} / \mathscr{D}_{o}$ comes from (iii)] is the $\mathscr{D}_{0}$-Atiyah algebra.

Clearly, both the Atiyah algebras and the do-algebras form categories, $\mathscr{A} \sim \rightarrow \mathscr{D}_{\mathscr{A}}, \mathscr{D} \sim \rightarrow \mathscr{A}_{\mathscr{Z}}$ are functors between them, and we have

Lemma. These functors are inverse to each other.

So the Atiyah algebras are the same as the do-algebras. We have $\mathscr{D}_{E}=\mathscr{D}_{\mathscr{A}_{E}}$. 1.1.4. Let $\mathscr{A}$ be an $R$-Atiyah algebra.

Definition. A connection $\nabla$ on $\mathscr{A}$ is an $\mathcal{O}_{X}$-linear map $\nabla: \mathscr{T}_{X} \rightarrow \mathscr{A}$ such that $\varepsilon \nabla=\mathrm{id}_{\mathscr{T}_{X}}$. A curvature $\mathrm{c}_{\nabla} \in \Omega^{2} \otimes_{\mathcal{O}_{X}} R$ is defined by the formula $\mathrm{c}_{\nabla}\left(\tau_{1} \wedge \tau_{2}\right)$ $=\left[\nabla\left(\tau_{1}\right), \nabla\left(\tau_{2}\right)\right]-\nabla\left(\left[\tau_{1}, \tau_{2}\right]\right)$. The connection $\nabla$ is integrable if $\mathrm{c}_{\nabla}=O$.

The connections of $\mathscr{A}$ form a $\operatorname{Hom}_{\mathscr{O}_{X}}\left(\mathscr{T}_{X}, R\right)=\Omega_{X}^{1} \otimes R$-torsor

To give an integrable connection is the same as to give a morphism of Atiyah algebras $\mathscr{A}_{\mathscr{O}_{X}} \rightarrow \mathscr{A}\left[\nabla\right.$ corresponds to a morphism $\left.\tau+f \mapsto \nabla(\tau)+f, \tau \in \mathscr{T}_{X}, f \in \mathcal{O}_{X}\right]$. Or it is the same as to give a $\mathscr{T}_{X}$-action on $R$ together with the isomorphism $\mathscr{A} \simeq \mathscr{T}_{X} \bowtie R$ (= the semi-direct product with respect to this action).

A connection $\nabla$ defines the $d$-derivative (that we will also denote $\nabla$ ) of the graded algebra $\Omega^{*} \otimes R, \nabla(\omega \otimes r)=d(\omega) \otimes r+\omega \cdot \nabla(r), \nabla(r)(\tau)=[\nabla(\tau), r]$, where $\omega \in \Omega, r \in R, \tau \in \mathscr{T}_{X}, \nabla(r) \in \Omega^{1} \otimes R$. We have $\nabla^{2}(*)=\mathrm{c}_{\nabla} *$.

A connection on $\mathscr{A}_{E}$ is the same as a usual connection on $E$.

\subsubsection{Standard Operations on Atiyah Algebras. These are the following ones.}

(i) Push forward $\varphi_{*}$. Let $\mathscr{A}$ be an $R$-Atiyah algebra, and $R^{\prime}$ an $\mathcal{O}_{X}$-algebra. Consider a pair $\varphi=\left(\varphi_{\mathscr{A}}, \varphi_{R}\right)$ of $\mathcal{O}_{X}$-linear Lie algebra maps $\varphi_{\mathscr{A}}: \mathscr{A} \rightarrow \operatorname{Der} R^{\prime}$, $\varphi_{R}: R^{\mathrm{Lie}} \rightarrow R^{\prime \text { Lie }}$. Assume that $\operatorname{ad} \circ \varphi_{R}=\left.\varphi_{\mathscr{A}}\right|_{R}$ and $\varphi_{\mathscr{A}}(a)(f)=\varepsilon(a)(f)$ for $a \in \mathscr{A}$, $f \in \mathcal{O}_{X} \rightarrow R^{\prime}$. Define the $R^{\prime}$-Atiyah algebra $\varphi_{*}(\mathscr{A})$ to be the semi-direct product $R^{\prime}>\triangleleft \mathscr{A}$ modulo the relations $\left(\varphi_{R}(a), O\right)=(O, a), a \in R$. One has canonical $\mathscr{O}_{X}$-linear Lie algebras map $\mathscr{A} \rightarrow \varphi_{*} \mathscr{A}$.

(ii) The product. If $\mathscr{A}_{i}$ are $R_{i}$-Atiyah algebras we get an $R_{1} \times R_{2}$-Atiyah algebra $\mathscr{A}_{1} \underset{\mathscr{T}_{X}}{\times} \mathscr{A}_{2}$.

(iii) The opposite algebra for an $R$-Atiyah algebra $\mathscr{A}$ is the $R^{0}$-Atiyah algebra $\mathscr{A}^{0}$ such that $\mathscr{D}_{\mathscr{A}^{0}}=\left(\mathscr{D}_{\mathscr{A}}\right)^{0}$; here $R^{0},\left(\mathscr{D}_{\mathscr{A}}\right)^{0}$ is $R, \mathscr{D}$ with reversed multiplication. Explicitly, $\mathscr{A}^{0}=\mathscr{A}$ as a sheaf, $[,]_{\mathscr{A}^{0}}=-[]_{\mathscr{A}}, \varepsilon_{\mathscr{A}^{0}}=-\varepsilon_{\mathscr{A}}$, and the left $\mathcal{O}_{X^{-}}$-module structure for $\mathscr{A}^{0}$ is the right one for $\mathscr{A}$.

(iv) Let $\mathscr{A}_{i}$ be $R_{i}$-Atiyah algebras. Define the $R_{1} \otimes R_{2}$-algebra $\mathscr{A}_{1} \otimes \mathscr{A}_{2}$ to be $\varphi_{\otimes *}\left(\mathscr{A}_{1} \times \mathscr{A}_{2}\right)$, where $\varphi_{\otimes R}\left(r_{1}, r_{2}\right)=r_{1} \otimes 1+1 \otimes r_{2}, \quad \varphi_{\otimes \mathscr{A}}\left(a_{1}, a_{2}\right)\left(r_{1} \otimes r_{2}\right)$ $=\left[a_{1}, r_{1}\right] \otimes r_{2}+r_{1} \otimes\left[a_{2}, r_{2}\right], r_{i} \in R_{i},\left(a_{1}, a_{2}\right) \in \mathscr{A}_{1} \underset{\mathscr{T}_{X}}{\times} \mathscr{A}_{2}$.

Lemma. Let $E_{i}$ be vector bundles. We have canonical isomorphisms

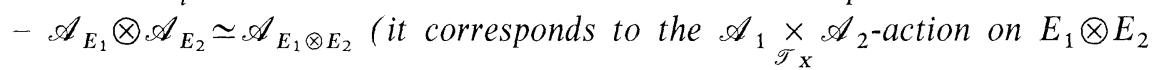
given by Leibnitz rule $\left.\left(\partial_{1}, \partial_{2}\right)\left(e_{1} \otimes e_{2}\right)=\partial_{1}\left(e_{1}\right) \otimes e_{2}+e_{1} \otimes \partial_{2}\left(e_{2}\right)\right)$. 
$-\mathscr{A}_{E}^{0} \simeq \mathscr{A}_{E^{0}}$, where $E^{0}:=\operatorname{Hom}\left(E, \omega_{X}\right), \omega_{X}:=\operatorname{det} \Omega_{X}^{1} \quad\left(\right.$ for $e \in E, \quad e^{0} \in E^{0}$, $\partial \in \mathscr{A}_{E}^{0}=\mathscr{A}_{E}$ one has $\left.\left(\partial e^{0}\right)(e)=e^{0}(\partial e)-\operatorname{Lie}_{\varepsilon_{, g}(\partial)}\left(e^{0}(e)\right)\right)$.

(v) $X$-functoriality. Let $\mathscr{A}$ be an $R$-Atiyah algebra on $X$. If $f: Y \rightarrow X$ is a morphism of smooth varieties, then we get $f^{*}(R)=f^{-1} R \underset{f^{-1} \mathscr{C}_{X}}{\otimes} \mathscr{O}_{Y}$-Atiyah algebra $f^{*}(\mathscr{A}):=\mathscr{T}_{Y} \underset{f^{*} \mathscr{T}_{X}}{\times} f^{*}(\mathscr{A})$. If $\pi: X \rightarrow Z$ is étale finite map, then $\pi \cdot(\mathscr{A}):=\mathscr{T}_{Z_{\pi_{*}}} \underset{\mathscr{T}_{X}}{\times} \pi_{*}(\mathscr{A})$ is $\pi_{*}(R)$-Atiyah algebra.

1.1.6. An important class of Atiyah algebras are $\mathfrak{O}_{X}$-Atiyah algebras. They form a groupoid which is a " $\mathbb{C}$-vector space in categories": the tensor product gives the sum of objects, and the multiplication by $\lambda \in \mathbb{C}$ is the push-forward by $\mathcal{O}_{X} \stackrel{\lambda \mathrm{id}}{\longrightarrow} \mathcal{O}_{X}$. The corresponding $\mathbb{C}$-vector space of isomorphism classes of objects is $H^{2}\left(X, \sigma_{\geq 1} \Omega_{X}^{\prime}\right)=H^{2}\left(X, 0 \rightarrow \Omega_{X}^{1} \rightarrow \Omega_{X}^{2} \rightarrow \Omega_{X}^{3}\right)$; this space coincides with $H_{\mathrm{DR}}^{2}(X)$ when $X$ is affine and with $F^{1} H_{\mathrm{DR}}^{2}(X)$ when $X$ is proper. Here is a Cech construction of the characteristic class $\mathrm{c}(\mathscr{A}) \in H^{2}\left(X, \sigma_{\geqq 1} \Omega_{X}\right)$ of an $\mathscr{O}_{X}$-Atiyah algebra $\mathscr{A}$ : for an open covering $U_{i}$ choose some connections $\nabla_{i}$ on $\left.\mathscr{A}\right|_{U_{i}}$; then $\mathrm{c}(\mathscr{A})=\left(\mathrm{c}_{\nabla_{i}}, \nabla_{i}-\nabla_{j}\right)$. The locally trivial $\mathcal{O}_{X}$-Atiyah algebras are just twisted differential operator rings from [3]. Note that the functor $L \mapsto \mathscr{A}_{L}$, where $L$ is an invertible sheaf, is a morphism of "groups in categories" (= Picard categories); on the level of isomorphism classes of objects this is just $\mathrm{c}_{1}$ in de Rham cohomology.

1.1.7. Traces and Determinants. Let $\mathscr{A}$ be an $R$-Atiyah algebra. A trace on $\mathscr{A}$ is an $\mathcal{O}_{X}$-linear map $\operatorname{Tr}: R \rightarrow \mathcal{O}_{X}$ such that $\operatorname{Tr}([a, r])=\varepsilon(a)(\operatorname{Tr} r)$ for $a \in \mathscr{A}, r \in R$. According to 1.1.5(i) we get an $\mathscr{O}_{X}$-Atiyah algebra $\operatorname{Tr}_{*}(\mathscr{A})$.

Example. Let $E$ be a vector bundle. The ordinary trace $\operatorname{tr}:$ End $E \rightarrow \mathcal{O}_{X}$ is a trace on $\mathscr{A}_{E}$. We have a canonical isomorphism

$$
\operatorname{tr}_{*}\left(\mathscr{A}_{E}\right) \simeq \mathscr{A}_{\operatorname{det} E}
$$

that corresponds to the action of $\mathscr{A}_{E}$ on $\operatorname{det} E$ given by the formula $\partial\left(e_{1} \wedge \ldots \wedge e_{n}\right)$ $=\sum_{i} e_{1} \wedge \ldots \wedge \partial\left(e_{i}\right) \wedge \ldots \wedge e_{n}$.

\section{2. $\pi$-Algebras, Integration Over the Fiber}

From now on $\pi: X \rightarrow S$ will be a smooth morphism of relative dimension 1 between smooth varieties (hence $X$ is a family of curves parametrised by $S$ ). We have a canonical exact sequence $0 \rightarrow \mathscr{T}_{X / S} \rightarrow \mathscr{T}_{X} \stackrel{d \pi}{\longrightarrow} \pi^{*} \mathscr{T}_{S} \rightarrow 0$, where $\mathscr{T}_{X / S}$ is the relative tangent bundle. The sheaf $\pi^{*} \mathscr{T}_{S}=\mathcal{O}_{X} \otimes \pi^{-1} \mathscr{T}_{S}$ contains the subsheaf $\pi^{-1} \mathscr{T}_{S}$. Put $\mathscr{T}_{\pi}:=(d \pi)^{-1}\left(\pi^{-1} \mathscr{T}_{S}\right) \subset \mathscr{T}_{X} ;$ clearly $\left[\mathscr{T}_{\pi}, \mathscr{T}_{\pi}\right] \subset \mathscr{T}_{\pi}, \pi^{-1} \mathcal{O}_{S} \cdot \mathscr{T}_{\pi} \subset \mathscr{T}_{\pi}$, and we have the exact sequence $0 \rightarrow \mathscr{T}_{X / S} \rightarrow \mathscr{T}_{\pi} \stackrel{d \pi}{\longrightarrow} \pi^{-1} \mathscr{T}_{S} \rightarrow 0$ of Lie algebras. Denote by $\mathscr{T}_{\pi}^{*}$ the differential graded (dg-for short) Lie superalgebra with $\mathscr{T}_{\pi}^{o}:=\mathscr{T}_{\pi}, \mathscr{T}_{\pi}^{-1}:=\mathscr{T}_{X / S}$, $\mathscr{T}_{\pi}^{i}=0$ for $i \neq 0,-1$, the differential $\mathscr{T}_{\pi}^{-1} \rightarrow \mathscr{T}_{\pi}^{0}$ is a canonical embedding, the bracket on $\mathscr{T}_{\pi}^{0}$ is the Lie one. Our $\mathscr{T}_{\pi}^{\cdot}$ has also a $\pi^{-1} \mathcal{O}_{\mathrm{S}}$-module structure together with the morphism $\bar{\varepsilon}: \mathscr{T}_{\pi}^{\cdot} \rightarrow \pi^{-1} \mathscr{T}_{S}\left(=H^{0}\left(\mathscr{T}_{\pi}^{*}\right), \bar{\varepsilon}=d \pi\right)$ of $\pi^{-1} \mathcal{O}_{S}$ modules compatible with brackets such that $[a, f b]=\bar{\varepsilon}(a)(f) b+f[a, b]$ for $f \in \pi^{-1} \mathcal{O}_{S}, a, b \in \mathscr{T}_{\pi}$.

Consider the relative de Rham complex $\Omega_{X / S}=\left(\mathscr{O}_{X} \rightarrow \omega\right), \omega:=\Omega_{X / S}^{1}$. It is naturally a dg $-\mathscr{T}_{\pi}^{\prime}$-module: the action of $\mathscr{T}_{\pi}^{*}$ is the Lie derivative on $\mathscr{T}_{\pi}^{0}$, and the 
obvious convolution $\mathscr{T}_{\pi}^{-1} \otimes \omega=\mathscr{T}_{X / S} \otimes \Omega_{X / S}^{1} \rightarrow \mathcal{O}_{X}$ on $\mathscr{T}_{\pi}^{-1}$. Also, $\Omega_{X / S}$ is a complex of $\pi^{-1} \mathcal{O}_{S}$-modules, and we have the obvious compatibility between $\pi^{-1} \mathcal{O}_{S}$ and $\mathscr{T}_{\pi}$. actions.

1.2.1. Definition. a) An $\mathscr{O}_{S}$-Lie algebra $\mathscr{A}^{\circ}$ on $X$ is a dg-Lie superalgebra together with a $\pi^{-1} \mathcal{O}_{S}$-module structure and a morphism $\varepsilon_{\mathscr{A}}: \mathscr{A}^{\cdot} \rightarrow \mathscr{T}_{\pi}$, compatible with the above structures such that $[a, f b]=\bar{\varepsilon}_{\mathscr{A}}(a)(f) b+f[a, b]$, where $\bar{\varepsilon}_{\mathscr{A}}:=\bar{\varepsilon} \circ \varepsilon_{\mathscr{A}}$.

b) A $\pi$-algebra $\mathscr{A}$ is an $\mathcal{O}_{S}$-Lie algebra together with a 3 step filtration $0=\mathscr{A}_{-3}$ $\subset \mathscr{A}_{-2} \subset \mathscr{A}_{-1} \subset \mathscr{A}_{0}=\mathscr{A}^{\cdot}$ and an isomorphism $\mathscr{A}_{-2}^{\cdot} \simeq \Omega_{X / S}^{\cdot}[2]$ of $\mathcal{O}_{\mathrm{S}}$-complexes such that

(i) $\left[\mathscr{A}_{i}, \mathscr{A}_{j}\right] \subset \mathscr{A}_{i+j}, \mathscr{O}_{S} \mathscr{A}_{i} \subset \mathscr{A}_{i}$.

(ii) $\mathscr{A}_{-1}^{\cdot} / \mathscr{A}_{-2}$ is acyclic.

(iii) $\varepsilon_{\mathscr{A}}: \mathscr{A}^{*} \rightarrow \mathscr{A}^{*} / \mathscr{A}_{-1}{ }_{-1} \underset{\rightarrow}{\rightarrow}$.

(iv) The ad action of $\mathscr{A}^{*} / \mathscr{A}_{-1}$ on $\mathscr{A}_{-2}^{\cdot}$ coincides with the above action of $\mathscr{T}_{\pi}$ on $\Omega_{X / S}^{\cdot}$.

We will say that $\mathscr{A}$ is an $\Omega$-extension of the $\mathcal{O}_{S}$-Lie algebra $\mathscr{A}^{\cdot} / \mathscr{A}_{-2}$.

Clearly the $\pi$-algebras form a category (the morphisms induce the identity maps on $\mathscr{T}_{\pi}$ and $\left.\Omega_{X / S}\right)$. For two $\pi$-algebras $\mathscr{A}$, $\mathscr{A}^{\prime}$ put $\mathscr{A}^{\prime} \times \mathscr{A}^{\prime \prime}:=\mathscr{A}_{\mathscr{T}_{\pi}} \times \mathscr{A}^{\prime \prime} /(\omega,-\omega) \in\left(\Omega_{X / S}^{*} \times \Omega_{X / S}^{*}\right)$ [2]: this is the $\Omega$-extension of $\mathscr{A} / \mathscr{A}_{-2} \underset{\mathscr{T}_{\pi}}{\times} \mathscr{A}^{\cdot} \mathscr{\mathscr { A }}_{-2}$. For a fixed $\mathscr{O}_{S}$-Lie algebra $\mathscr{B}$ consider the category of $\Omega$-extensions of $\mathscr{B}$. This is a groupoid which is a $\mathbb{C}$-vector space in categories with the sum $\mathscr{A}^{\cdot}+\mathscr{A}^{\prime}:=\mathscr{A}^{*} \times \mathscr{A}^{\prime} /(\omega,-\omega), \omega \in \Omega_{X / S}$ [2] (this is a sub $\pi$-algebra of $\left.\mathscr{A}^{*} \times \mathscr{A}^{\prime}\right)$ and multiplication by $\lambda \in \mathbb{C}$ defined as $\lambda \cdot \mathscr{A}^{*}=$ push forward of $\mathscr{A}^{\cdot}$ by $\lambda \cdot \mathrm{id}_{\Omega}: \Omega^{\prime}[2] \rightarrow \Omega \cdot[2]$.

If $S^{\prime} \stackrel{\varphi}{\longrightarrow} S$ is a morphism of smooth varieties, we have an obvious base change functors $\varphi^{*}$ from $\mathcal{O}_{S}$-Lie algebras, or $\pi$-algebras, on $X$, to the ones on $X \times S_{S}^{\prime}$, compatible with the above operations.

1.2.2. We will use $\pi$-algebras that are related to Atiyah algebras. So let $\mathscr{A}$ be an $R$ Atiyah algebra on $X$. Put $\mathscr{A}_{/ S}:=\varepsilon_{\mathscr{A}}^{-1}\left(\mathscr{T}_{X / S}\right) \subset \mathscr{A}_{\pi}:=\varepsilon_{\mathscr{A}}^{-1}\left(\mathscr{T}_{\pi}\right) \subset \mathscr{A}$. Clearly $\mathscr{A}_{\pi}$ is a Lie subalgebra in $\mathscr{A}, \mathscr{A}_{S}$ an ideal in $\mathscr{A}_{\pi}, \mathscr{A}_{S}\left(\mathscr{A}_{\pi}\right)$ an $\mathcal{O}_{X^{-}}\left(\right.$respectively $\left.\pi^{-1} \mathscr{O}_{S}\right)$ submodule of $\mathscr{A}$. Denote by $\mathscr{A}_{\pi}^{*}$ an $\mathscr{O}_{\mathrm{S}}$-Lie algebra with $\mathscr{A}_{\pi}^{0}:=\mathscr{A}_{\pi}, \mathscr{A}_{\pi}^{-1}:=\mathscr{A}_{\mathrm{S}}$, $\mathscr{A}^{i}=0$ for $i \neq 0,-1$, the differential $\mathscr{A}_{\pi}^{-1} \rightarrow \mathscr{A}_{\pi}^{0}$ is inclusion $\mathscr{A}_{/ S} \rightarrow \mathscr{A}_{\pi}$, the bracket comes from $\mathscr{A}$, and the projection $\varepsilon_{\mathscr{A}_{\pi}}: \mathscr{A}_{\pi} \rightarrow \mathscr{T}_{\pi}$ is $\varepsilon_{\mathscr{A}}$. Thus $\varepsilon_{\mathscr{A}_{\pi}}$ is surjective and $\operatorname{Ker} \varepsilon_{\mathscr{A}_{\pi}}$ is Cone $\left(\mathrm{id}_{R}\right)$.

Definition. An $\Omega$-extension ${ }^{\#} \mathscr{A}^{\cdot}$ of $(\mathscr{A}, R)$, is an $\Omega$-extension of $\mathscr{A}_{\pi}^{\cdot}$ together with an $\mathcal{O}_{X}$-module structure on $\# \mathscr{A}^{-1}$ such that

- this $\mathcal{O}_{X}$-action is compatible with the actions on $\mathscr{A}_{\pi}^{-1}=\mathscr{A}_{\mid S}$ and $\omega$.

- the component $[,]_{-1,-1}:{ }^{\#} \mathscr{A}^{-1} \otimes{ }^{\#} \mathscr{A}^{-1} \rightarrow{ }^{\#} \mathscr{A}^{-2}=\mathcal{O}_{X}$ is a differential operator along the fibers (i.e. for any $a \in{ }^{\#} \mathscr{A}^{-1}$ the $\operatorname{map~ad}_{a}:{ }^{\#} \mathscr{A}^{-1} \rightarrow \mathcal{O}_{X}$ is a d.o.).

Remark. $[,]_{-1,-1}$ is completely determined by $[,]_{0,-1}:{ }^{\#} \mathscr{A}^{0} \otimes \mathscr{A}^{-1} \rightarrow \mathscr{A}^{-1}$ (since any two $[,]_{-1,-1}$ 's differ by a map \# $\mathscr{A}^{-1} \otimes \mathbb{A}^{-1} \rightarrow \pi^{-1} \mathcal{O}_{S}$ $=\operatorname{Ker}\left(d: \mathcal{O}_{X} \rightarrow \omega\right)$ which has to be zero being a differential operator).

The $\Omega$-extensions of Atiyah algebras form a category; we have a forgetting functor on it with values in $\pi$-algebras. The standard operations $X,+, \lambda$ on $\pi$-algebras extend literally to the present situation. 
1.2.3. Assume that $\pi$ is proper. Let $\mathscr{A}$ be a $\pi$-algebra. Then $R^{0} \pi_{*}$, applied to $\Omega_{X / S}[2] \rightarrow \mathscr{A}^{\circ} \rightarrow \mathscr{T}_{\pi}^{\prime}$, together with the trace isomorphism $R^{0} \pi_{*} \Omega_{X / S}^{\prime}[2] \sim \mathcal{O}_{S}$, gives the exact sequence

$$
0 \rightarrow \mathcal{O}_{S} \rightarrow R^{0} \pi_{*} \mathscr{A} \rightarrow \mathscr{T}_{S} \rightarrow 0 .
$$

The bracket and $\pi^{-1} \mathcal{O}_{S}$-action on $\mathscr{A}$ define the Lie algebra and $\mathcal{O}_{S}$-module structure on $R^{0} \pi_{*} \mathscr{A}$. It is easy to see that they form an $\mathcal{O}_{\mathrm{S}}$-Atiyah algebra structure on $R^{0} \pi_{*} \mathscr{A}$; denote this algebra by $\pi(\mathscr{A})$.

Remark. If the fibers of $\pi$ are not connected, then $R^{0} \pi_{*} \mathscr{A} \cdot$ is a $\pi_{*} \mathcal{O}_{X}$-Atiyah algebra, and $\pi(\mathscr{A}):=\operatorname{tr} R^{0} \pi_{*} \mathscr{A}$, where $\operatorname{tr}: \pi_{*} \mathscr{O}_{X} \rightarrow \mathcal{O}_{S}$ is the trace map.

This way we get the "integration along the fibers" functor from $\pi$-algebras to $\mathcal{O}_{S}$-Atiyah algebras. It is compatible with base change, and we have $\pi\left(\mathscr{A} \times \mathscr{A}^{\prime \prime}\right)$ $=\pi\left(\mathscr{A}^{\prime}\right)+\pi\left(\mathscr{A}^{\prime}\right), \pi\left(\mathscr{A}{ }^{\prime}+\mathscr{A}^{\prime}\right)=\pi(\mathscr{A})+\pi\left(\mathscr{A}^{\prime \prime}\right), \pi(\lambda \mathscr{A})=\lambda \pi\left(\mathscr{A}^{\prime}\right)$.

\subsection{Central Extensions of Atiyah Algebras}

Here we will work in Zariski topology, at least fiberwise.

1.3.1. Let $\mathscr{A}^{\circ}$ be any dg-Lie superalgebra. Put $V(\mathscr{A}):=\mathscr{A}^{-1} / \mathscr{A}^{-2}$. Define the new bracket []$_{V}$ on $V(\mathscr{A})$ by the formula $[\alpha, \beta]_{V}:=[d \alpha, \beta]$. Let $J$ denote the image of the differential $d: \mathscr{A}^{-1} \rightarrow \mathscr{A}^{0}$; this is a subalgebra of $\mathscr{A}^{0}$. We have $d\left([\alpha, \beta]_{V}\right)=[d \alpha, d \beta]$.

Lemma. $[,]_{V}$ is a Lie bracket; $V(\mathscr{A})$ with $[,]_{V}$ is a central extension of $J$ by $H^{-1}(\mathscr{A})$.

1.3.1. Let $\mathscr{A}$ be any dg-Lie superalgebra. Put $V(\mathscr{A}):=\mathscr{A}^{-1} / d \mathscr{A}^{-2}$. Define the construction gives us a central extension of $\mathscr{A}_{S}$ by the $\pi^{-1} \mathscr{O}_{S}$-sheaf $\mathscr{H}=\mathscr{H}_{X / S}^{1}:=\omega / d\left(\mathcal{O}_{X}\right)$. In the case $S=$ point we have $\mathscr{H}(U)=H_{\mathrm{DR}}^{1}(U)$ for a (Zariski) open $j: U \hookrightarrow X$, and there is a canonical exact sequence

$$
0 \rightarrow H_{\mathrm{DR}}^{1}(X) \stackrel{j^{*}}{\longrightarrow} H_{\mathrm{DR}}^{1}(U) \stackrel{\text { res }}{\longrightarrow} \mathbb{C}^{n} \stackrel{\Sigma}{\longrightarrow} \mathbb{C} \rightarrow 0,
$$

where $\operatorname{res}(v)=\left(\operatorname{res}_{x_{i}}(v)\right), \Sigma\left(a_{1}, \ldots, a_{n}\right)=a_{1}+\ldots+a_{n}$, and $\left\{x_{1}, \ldots, x_{n}\right\}:=X \backslash U$.

Clearly, $V\left(\left(^{\#} \mathscr{A}^{\prime}\right) \rightarrow \mathscr{A}_{\pi}\right.$ is a dg-Lie superalgebra quotient of ${ }^{\#} \mathscr{A}$, and $\pi\left({ }^{\#} \mathscr{A}^{*}\right)$ $=R^{0} \pi_{*}\left(V\left({ }^{\#} \mathscr{A}^{*}\right) \rightarrow \mathscr{A}_{\pi}\right)$.

The rest of this subsection will be used only in Appendix, A5.

1.3.3.1. Definition. A $\tilde{\pi}$-algebra $\tilde{\mathscr{A}}$ on $X$ is an $\mathcal{O}_{S}$-Lie algebra together with three step filtration $0=\tilde{\mathscr{A}}_{-3} \subset \tilde{\mathscr{A}}_{-2} \subset \tilde{\mathscr{A}}_{-1} \subset \mathscr{A}_{0}=\mathscr{A}$ and the isomorphism $\mathscr{A}_{-2} \simeq\left(\mathcal{O}_{X} \rightarrow \omega \rightarrow \mathscr{H}\right)$ of $\pi^{-1} \mathcal{O}_{S}$-complexes $(\mathscr{H}$ stands in degree $0, \omega \rightarrow \mathscr{H}$ is the canonical projection) such that the properties 1.2.1.b (i)-(iv) hold.

Clearly, $\mathscr{H} C \tilde{\mathscr{A}}_{-2}^{0} \subset \tilde{\mathscr{A}}^{\cdot}$ is an abelian ideal, and ${ }^{\#} \mathscr{A}^{*}:=\tilde{\mathscr{A}}^{\cdot} \mid \mathscr{H}$ is a $\pi$-algebra. We will say that $\tilde{\mathscr{A}}^{\prime}$ is an $\mathscr{H}$-extension of $\mathscr{A}^{\circ}$. We have ${ }^{\#} \mathscr{A}^{i}=\tilde{\mathscr{A}}^{i}$ for $i \neq 0$, and $\mathscr{A}^{0}$ is the $\mathscr{H}$-extension of ${ }^{\#} \mathscr{A}^{0}$ that coincides with $V(\mathscr{A})$ over $J C^{\#} \mathscr{A}^{0}$.

The projection $\varepsilon_{\tilde{\mathscr{A}}}: \tilde{\mathscr{A}}_{\rightarrow} \mathscr{T}_{\pi}$ defines the $\mathcal{O}_{S}$-linear isomorphism $R^{0} \pi_{*}\left(\varepsilon_{\mathscr{A}}\right): R^{0} \pi_{*} \tilde{\mathscr{A}}^{\cdot} \rightarrow R^{0} \pi_{*} \mathscr{T}_{\pi}=\mathscr{T}_{S}$ of Lie algebras. Hence $\tilde{\mathscr{A}}$ defines the integrable connection $\nabla_{\mathscr{A}}=R^{0} \pi_{*}(l)\left(R^{0} \pi_{*}\left(\varepsilon_{\mathscr{A}}\right)\right)^{-1}: \mathscr{T}_{S} \rightarrow \pi\left({ }^{\#} \mathscr{A}\right)$ on $\left.\pi^{*} \mathscr{A}^{*}\right)$; here $l: \tilde{\mathscr{A}}^{\rightarrow}{ }^{\#} \mathscr{A}^{\cdot}$ is the projection. 
Let $\widetilde{\Omega}_{S}^{c l} \subset \pi^{-1} \Omega_{S}^{1} \otimes_{\pi^{-1} \mathscr{O}_{S}} \mathscr{H}$ be the sheaf of closed $\mathscr{H}$-valued 1 -forms (recall that $\mathscr{H}$ has a natural $\pi^{-1} \mathscr{T}_{S}$-action). For any $\tilde{\pi}$-algebra $\tilde{\mathscr{A}}$ we may identify $\widetilde{\Omega}_{S}^{c l}$ with the sheaf of automorphisms of $\tilde{\mathscr{A}}$ that induce the identity transformation on ${ }^{\#} \mathscr{A}^{*}$ : a form $v \in \widetilde{\Omega}_{S}^{c l}$ acts by the formula $a \in \tilde{\mathscr{A}}^{\cdot} \mapsto a+v \bar{\varepsilon}(a)$. Hence the $\mathscr{H}$-extensions of \# $\mathscr{A}^{\text {. }}$ that are locally isomorphic to $\tilde{\mathscr{A}}^{\cdot}$ are in $1-1$ correspondence with $\widetilde{\Omega}_{S}^{\text {cl }}$-torsors; for $\tilde{v} \in H^{1}\left(X, \widetilde{\Omega}_{S}^{c l}\right)$ denote by $\tilde{\mathscr{A}}^{\cdot}+\tilde{v}$ the corresponding isomorphism class of $\mathscr{H}$-extensions. We have a trace map $H^{1}\left(X, \widetilde{\Omega}_{S}^{c l}\right) \rightarrow \Omega_{S}^{1 c l}$. It is easy to see that $\nabla_{\tilde{A}+\tilde{v}}$ $=\nabla_{\mathscr{A}}+\operatorname{tr} \tilde{v}$.

\section{Trace Algebras and Atiyah Algebra of a Determinant Bundle}

\subsection{Trace $\Omega$-Extension of $\mathscr{A}_{E}$}

Assume we are in the situation of 1.2 .

2.1.1. Residues at the Diagonal. Let $P_{1}, P_{2}: X \underset{S}{\times} X \rightarrow X$ be projections, $\Delta: X \hookrightarrow X \times X$ the diagonal; the sheaves on $X \underset{S}{\times} X$ with support at $\Delta(X)$ will be identified with sheaves on $X$ via $A^{-1}$. Put $\mathcal{O}_{\hat{A}}=\underbrace{\lim }_{i} \mathcal{O}_{X \times X} / \mathcal{O}_{X \times X}(-i \Delta)$.

2.1.1.1. Consider the sheaf $\omega 区 \omega(\infty \Delta)$ on $X \underset{S}{\times} X$. We have two residue maps $\operatorname{Res}^{1}, \operatorname{Res}^{2}: \omega \bowtie \omega(\infty \Delta) \rightarrow \omega$, where $\operatorname{Res}^{1}\left(\operatorname{Res}^{2}\right)$ is the residue along the first (second) coordinate with the other one fixed.

The formula: let $t$ be a coordinate along the fiber, $t_{1}, t_{2}$ the corresponding coordinates on $X \times X, f\left(t_{1}, t_{2}, s\right) \in \mathbb{C}_{X \times X}, \quad \varphi=f\left(t_{1}, t_{2}, s\right)\left(t_{1}-t_{2}\right)^{-i-1} d t_{1} d t_{2}$ $\in \omega 区 \omega((i+1) \Delta)$, then

$$
\begin{aligned}
& \operatorname{Res}^{1}(\varphi)=\left.\frac{1}{i !} \frac{\partial^{i} f}{\partial t_{1}^{i}}\left(t_{1}, t, s\right)\right|_{t_{1}=t} d t, \\
& \operatorname{Res}^{2}(\varphi)=\left.\frac{(-1)^{i+1}}{i !} \frac{\partial^{i} f}{\partial t_{2}^{i}}\left(t, t_{2}, s\right)\right|_{t_{2}=t} d t .
\end{aligned}
$$

Clearly Res ${ }^{i}$ maps $\omega \bowtie \omega \subset \omega \bowtie \omega(\infty \Delta)$ into zero.

For $\varphi$ as above put

$$
(\tilde{\operatorname{Res}} \varphi)(t, s)=\left.\frac{1}{i !} \sum_{a+b=i-1}(-1)^{b} \frac{\partial^{i-1} f\left(t_{1}, t_{2}, s\right)}{\partial t_{1}^{a} \partial t_{2}^{b}}\right|_{t_{1}=t_{2}=t} \in \mathcal{O}_{X} .
$$

Lemma. a) Res is a correctly defined map $\omega \bowtie \omega(\infty \Delta) \rightarrow \mathcal{O}_{X}$ (i.e. Res does not depend on the choice of coordinates).

b) One has $d \tilde{\operatorname{Res}}(\varphi)=\operatorname{Res}^{1}(\varphi)-\operatorname{Res}^{2}(\varphi)$.

c) Res vanishes on $\omega 冈 \omega(\Delta)$, and is symmetric with respect to transposition of coordinates.

Proof. b), c) are clear; b) $\Rightarrow$ a), since Res may be characterised as a unique differential operator (along the fibers) such that $d \tilde{\operatorname{Res}}=\operatorname{Res}^{1}-\operatorname{Res}^{2}$ (if $\tilde{\operatorname{Res}}, \tilde{\operatorname{Res}^{\prime}}$ are two such operators, then Res $-\widetilde{R_{e}} s^{\prime}$ maps $\varphi$ 's into constants $\pi^{-1} \mathcal{O}_{S}$, but any such differential operator is zero). 
2.1.1.2. For a pair of integers $a, b, a \geqq b$, put

$$
\begin{gathered}
\mathscr{P}_{a b}=\mathcal{O}_{X} \boldsymbol{O}_{S} \omega((a+1) \Delta) / \mathcal{O}_{X} \bowtie \omega((b+1) \Delta), \\
\mathscr{P}=\mathscr{P}_{\infty,-\infty}=\lim _{\longleftarrow} \mathcal{O}_{X} \bowtie \omega(\infty \Delta) / \mathcal{O}_{X} \bowtie \omega(-i), \mathscr{P}_{i}:=\mathscr{P}_{i,-\infty} \subset \mathscr{P} .
\end{gathered}
$$

The sheaf $\mathscr{P}$ has a natural $\mathscr{D}_{X / S^{-}}$bimodule structure [for $\partial=A(t) \partial_{t}+B(t)$, $\psi=\psi\left(t_{1}, t_{2}\right) d t_{2}$, one has $\partial \psi=\left(A\left(t_{1}\right) \partial_{t_{1}}+B\left(t_{1}\right)\right) \psi\left(t_{1}, t_{2}\right) d t_{2}, \quad \psi \partial=\left(\psi\left(t_{1}, t_{2}\right) B\left(t_{2}\right)\right.$ $\left.\left.-\partial_{t_{2}}\left(\psi\left(t_{1}, t_{2}\right) A\left(t_{2}\right)\right) d t_{2}\right)\right]$; $\mathscr{P}_{-1} \subset \mathscr{P}$ is a subbimodule, and one has canonical isomorphism "pole at $\Delta$ ": $\delta: \mathscr{P} / \mathscr{P}_{-1} \rightarrow \mathscr{D}_{X / S}, \quad \delta \psi(f)=\operatorname{Res}_{t_{2}=t} \psi\left(t, t_{2}\right) f\left(t_{2}\right) d t_{2}$ for $\psi=\psi\left(t_{1}, t_{2}\right) d t \in \mathscr{P}, f \in \mathcal{O}_{X}$.

For a vector bundle $E$ put $\mathscr{P}_{E, a, b}:=E \otimes_{\mathcal{O}_{X}} \mathscr{P}_{a b} \otimes_{\mathcal{O}_{X}} E^{*}, \mathscr{P}_{E}=\ldots ; \mathscr{P}_{E}$ is a $\mathscr{D}_{E / S}$ bimodule, and $\delta_{E}: \mathscr{P}_{E} / \mathscr{P}_{E,-1} \rightarrow \mathscr{D}_{E / S}$ is isomorphism. Let $\mathscr{D}_{E / S}^{\text {Lie }}$ denote $\mathscr{D}_{E / S}$ considered as a Lie algebra $\left(\left[\partial_{1}, \partial_{2}\right]=\partial_{1} \partial_{2}-\partial_{2} \partial_{1}\right)$.

The bimodule structure determines a $\mathscr{D}_{E / S}^{\text {Lie }}$-action on $\mathscr{P}_{E}$ by the formula $\operatorname{Lie}(\partial)(\psi)=\partial \psi-\psi \partial, \partial \in \mathscr{D}_{E / S}, \psi \in \mathscr{P}_{E}$. In fact this action extends to an action of the Lie subalgebra $\mathscr{A}_{E, \pi}+\mathscr{D}_{E / S}^{\mathrm{Li}} \subset \mathscr{D}_{E}^{\mathrm{Lie}}$ [where $\mathscr{A}_{E, \pi}$ acts as Lie algebra of infinitesimal symmetries of $(X, E, S, \pi)]$. The transposition of coordinates ${ }^{t}$ defines an isomorph-

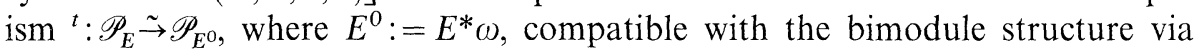
isomorphism $\mathscr{D}_{E / S}^{0}=\mathscr{D}_{E^{0} / S}$.

2.1.1.3. Define the pairing $\langle\cdot, \cdot\rangle: \mathscr{P} \otimes \mathscr{P} \rightarrow \mathcal{O}_{X}$ by the formula $\left\langle\psi_{1}, \psi_{2}\right\rangle$ $=\tilde{\operatorname{Res}}\left(\psi_{1}{ }^{t} \psi_{2}\right)$. We have $\left\langle\psi_{1}, f \psi_{2}\right\rangle=\left\langle\psi_{1} f, \psi_{2}\right\rangle$ for $f \in \mathcal{O}_{X},\left\langle\psi_{1}, \psi_{2}\right\rangle=\left\langle\psi_{2}, \psi_{1}\right\rangle$, $\left\langle\psi_{1}, \psi_{2}\right\rangle=0$ for $\psi_{1} \in \mathscr{P}_{a+1}, \psi_{2} \in \mathscr{P}_{-a}$. The same formula (joined with convolution $\left.E \otimes E^{0} \rightarrow \omega\right)$ defines the pairing $\langle\cdot, \cdot\rangle_{E}: \mathscr{P}_{E} \otimes \mathscr{P}_{E} \rightarrow \mathcal{O}_{X}$ which is $\mathscr{A}_{E}$-invariant. Let tr denote the composition $\mathscr{P}_{E,-1} \rightarrow \mathscr{P}_{E,-1} / \mathscr{P}_{E,-2}=\omega \cdot$ End $E \stackrel{\text { tr }}{\longrightarrow} \omega$.

Clearly, Lie $\delta\left(\psi_{1}\right)\left(\psi_{2}\right)+\operatorname{Lie} \delta\left(\psi_{2}\right)\left(\psi_{1}\right) \in \mathscr{P}_{E,-1}$ for $\psi_{1}, \psi_{2} \in \mathscr{P}_{E,-1}$ and we have

$$
\operatorname{tr}\left(\operatorname{Lie} \delta\left(\psi_{1}\right)\left(\psi_{2}\right)+\operatorname{Lie} \delta\left(\psi_{2}\right)\left(\psi_{1}\right)\right)=d\left\langle\psi_{1}, \psi_{2}\right\rangle_{E} .
$$

2.1.2. Construction of Trace $\Omega$-Extension. For a bundle $E$ define an $\Omega$-extension ${ }^{\operatorname{tr}} \mathscr{A}_{E}$ of $\mathscr{A}_{E}$ (see 1.2.2) - the trace algebra of $E$ - as follows. We have ${ }^{\operatorname{tr}} \mathscr{A}_{E}^{0}=\mathscr{A}_{E, \pi}$, ${ }^{\operatorname{tr}} \mathscr{A}_{E}^{-2}=\mathcal{O}_{X}$. To define ${ }^{\operatorname{tr}} \mathscr{A}_{E}^{-1}$ consider the exact sequence $0 \rightarrow \mathscr{P}_{E,-1}$ $\rightarrow \mathscr{P}_{E} \stackrel{\delta}{\rightarrow} \mathscr{D}_{E / S} \rightarrow 0$. Now use the embedding $\mathscr{A}_{E / S} \rightarrow \mathscr{D}_{E / S}$ and the trace $\operatorname{tr}: \mathscr{P}_{E,-1} \rightarrow \omega$ to push this sequence into

$$
0 \rightarrow \omega \rightarrow{ }^{\mathrm{tr}} \mathscr{A}_{E}^{-1} \rightarrow \mathscr{A}_{E / S} \rightarrow 0 .
$$

Hence ${ }^{\operatorname{tr}} \mathscr{A}_{E}^{-1}$ is the subquotient of $E \bowtie E^{0}(2 \Delta) / E 区 E^{0}(-\Delta)$ that consists of sections that map into $\mathscr{A}_{E / S}$ modulo the traceless ones in $($ End $E) \otimes \omega$. It has the natural (left) $\mathcal{O}_{X}$-action.

The above projection ${ }^{\text {tr }} \mathscr{A}_{E}^{-1} \rightarrow \mathscr{A}_{E / S}=\mathscr{A}_{E}^{-1}$ determines the projection ${ }^{\operatorname{tr}} \mathscr{A}_{E} \rightarrow \mathscr{A}_{E}$ and also the differential on ${ }^{\operatorname{tr}} \mathscr{A}_{E}$.

Brackets. The bracket $[\cdot, \cdot]_{0.0}:{ }^{\operatorname{tr}} \mathscr{A}_{E}^{0} \otimes{ }^{\operatorname{tr}} \mathscr{A}_{E}^{0} \rightarrow{ }^{\operatorname{tr}} \cdot \mathscr{A}_{E}^{0}$ should be the usual one; $[\cdot, \cdot]_{0,-1}$ is the Lie action of $\mathscr{A}_{E, \pi}$ on the (invariant) subquotient of $\mathscr{P}_{E}$ (see 2.1.1.2); $[\cdot, \cdot]_{0,-2}$ comes from $\varepsilon: \mathscr{A}_{E, \pi} \rightarrow \mathscr{T}_{\pi} ;[\cdot, \cdot]_{-1,-1}:{ }^{\operatorname{tr}} \mathscr{A}_{E}^{-1} \otimes{ }^{\operatorname{tr}} \mathscr{A}_{E}^{-1} \rightarrow \mathcal{O}_{X}$ is the pairing $\langle\cdot, \cdot\rangle_{E}$ frim 2.1.1.3 on the subquotient on $\mathscr{P}_{E}$. Now 2.1.1.3 implies

Lemma. The algebra ${ }^{\text {tr }} \mathscr{A}_{E}$ defined above is an $\Omega$-extension of $\mathscr{A}_{E}$.

Remarks. a) One may construct the trace extension for an arbitrary Atiyah algebra with trace, see Appendix, A2.

b) Clearly $E \sim \rightarrow^{\mathrm{tr}} \mathscr{A}_{E}^{\prime}$ is compatible with base change. 
Formulas: Let $t$ be a local coordinate (along the fiber), and $I: \mathcal{O}_{X}^{n} \stackrel{\sim}{\rightarrow} E$ a trivialisation of $E$; we will call the pair $(t, I)$ a gauge for $(X, E)$. It determines the isomorphisms

$$
\begin{aligned}
& s_{(t, I)}^{-1}: \mathcal{O}_{X} \oplus \operatorname{Mat}_{n}\left(\mathcal{O}_{X}\right) \oplus \mathcal{O}_{X} \stackrel{\sim}{\rightarrow}^{\operatorname{tr}} \mathscr{A}_{E}^{-1}, s_{(t, I)}^{-1}(\chi, B, v) \\
& =(\chi, B, v)_{(t, I)}=I\left[\frac{\chi\left(t_{1}\right)}{\left(t_{2}-t_{1}\right)^{2}}+\frac{B\left(t_{1}\right)}{t_{2}-t_{1}}+v\left(t_{1}\right)\right] d t_{2}, \\
& s_{I}^{0}: \mathscr{T}_{\pi} \oplus \operatorname{Mat}_{n}\left(\mathcal{O}_{X}\right) \stackrel{\sim}{\rightarrow}^{\mathrm{tr}} \mathscr{A}_{E}^{0}=\mathscr{A}_{E, \pi}, s_{I}^{0}(\tau, A)=(\tau, A)_{I} \text {. }
\end{aligned}
$$

We have [here $\tau=\tau(t, s) \partial_{t}+\mu(s) \partial_{s}$; we write for brevity $\chi^{\prime}=\partial_{t}(\chi(t, s))$, $\tau^{\prime}=\partial_{t} \tau(t, s)$, etc. $]$.

The Formulas. Brackets:

$$
\begin{aligned}
& {\left[(\tau, A)_{I},(\chi, B, v)_{(t, I)}\right]_{0,-1}=\left(\left[\tau, \chi \partial_{t}\right] d t, \tau(B)-\chi A^{\prime}\right.} \\
& \left.\quad-[A, B], \operatorname{Tr}\left(-B A^{\prime}+\frac{1}{2}\left(B \tau^{\prime \prime}-\chi A^{\prime \prime}\right)+\frac{1}{6} \chi \tau^{\prime \prime \prime}\right)+\operatorname{Lie}(\tau)(v d t) \partial_{t}\right), \\
& {\left[\left(\chi_{1}, B_{1}, v_{1}\right)_{(t, I)},\left(\chi_{2}, B_{2}, v_{2}\right)_{(t, I)}\right]_{-1,-1}=\operatorname{Tr}\left(-B_{1} B_{2}+\frac{1}{2}\left(\chi_{2}^{\prime} B_{1}\right.\right.} \\
& \left.\left.\quad+\chi_{1}^{\prime} B_{2}-\chi_{1} B_{2}^{\prime}-\chi_{2} B_{1}^{\prime}\right)+\frac{1}{6}\left(\chi_{1}^{\prime \prime} \chi_{2}-\chi_{1}^{\prime} \chi_{2}^{\prime}+\chi_{1} \chi_{2}^{\prime \prime}\right)\right)+\chi_{1} v_{2}+v_{1} \chi_{2} .
\end{aligned}
$$

Gauge Change: for $g \in \mathrm{GL}_{n}\left(\mathrm{O}_{X}\right)$ one has

$$
\begin{aligned}
& (\tau, A)_{I g}=\left(\tau,-\tau(g) g^{-1}+g A g^{-1}\right)_{I},(\chi, B, v)_{(t, I g)} \\
& \quad=\left(\chi,-\chi g^{\prime} g^{-1}+g B g^{-1}, \operatorname{Tr}\left(-\frac{1}{2} \chi g^{\prime \prime} g^{-1}+\chi\left(g^{\prime} g^{-1}\right)^{2}-B g^{-1} g^{\prime}\right)+v\right)_{(t, I)}
\end{aligned}
$$

if $y=y(t)$ is another coordinate function, then

$$
(\chi, B, v)_{(y, I)}=\left(\chi y^{\prime-1}, B, n \chi\left(\frac{1}{6} \frac{y^{\prime \prime \prime}}{y^{\prime 2}}-\frac{1}{4} \frac{y^{\prime \prime 2}}{y^{\prime 3}}\right)+\frac{1}{2} \frac{y^{\prime \prime}}{y^{\prime}}, \operatorname{Tr} B+v y^{\prime}\right)_{(t, I)} .
$$

Proof is a direct calculation. Clearly, these formulas completely define ${ }^{\operatorname{tr}} \mathscr{A}_{E}$.

\subsection{Virasoro Algebras}

In this subsection, and the next one, we assume that $S=$ point for brevity. Consider the Lie algebra $\hat{\mathscr{A}}_{E}:=V\left({ }^{\mathrm{tr}} \mathscr{A}_{E}\right)$ the central extension of $\mathscr{A}_{E}$ by $\mathscr{H}$ (we work in Zariski topology, see 1.3.2) and call it the Virasoro algebra of $E$. For a gauge $(t, I)$ we have an isomorphism

$$
S_{(t, I)}: \mathcal{O}_{X} \oplus \operatorname{Mat}_{n}\left(\mathcal{O}_{X}\right)+\mathcal{O}_{X} / \partial_{t}\left(\mathcal{O}_{X}\right) \stackrel{\sim}{\rightarrow} \hat{\mathscr{A}}_{E / S},
$$

$s_{(t, I)}(a)=a_{(t, I)}$, so that

$$
\begin{aligned}
& {\left[\left(\chi_{1} B_{1}, v_{1}\right)_{(t, I)},\left(\chi_{2}, B_{2}, v_{2}\right)_{(t, I)}\right]_{V}=\left(\chi_{1} \chi_{2}^{\prime}-\chi_{2} \chi_{1}^{\prime}, \chi_{1} B_{2}^{\prime}-\chi_{2} B_{1}^{\prime}+\left[B_{1}, B_{2}\right],\right.} \\
& \quad \operatorname{Tr}\left(B_{1} B_{2}^{\prime}+\frac{1}{2}\left(\chi_{2}^{\prime} B_{1}^{\prime}-\chi_{1}^{\prime} B_{2}^{\prime}\right)++\frac{1}{12}\left(\chi_{1}^{\prime} \chi_{2}^{\prime \prime}-\chi_{2}^{\prime} \chi_{1}^{\prime \prime}\right)\right)_{(t, I)},
\end{aligned}
$$

(see 2.1.2). So we see that for $X=\mathbb{C} \backslash\{0\}$ (here $\mathscr{O}_{X} / \partial_{t} \mathcal{O}_{X}=\mathbb{C}$ ) the restriction of $\hat{\mathscr{A}}_{\mathbb{C}^{n}}$ on $\operatorname{End}\left(\mathcal{O}_{X}^{n}\right)=\operatorname{Mat}_{n}\left(\mathcal{O}_{X}\right)$ coincides with an affine, or Kac-Moody, algebra, and the restriction on $\mathscr{T}_{X}$ coincides with the usual Virasoro algebra (hence the name). 
In fact, $\hat{\mathscr{A}}_{E}$ comes from the central extension $\widehat{\mathscr{D}}_{E}$ of $\mathscr{D}_{E}^{\text {Lie }}$ by $\mathscr{H}$ (via $\left.\mathscr{A}_{E} \hookrightarrow \mathscr{D}_{E}^{\mathrm{Lie}}\right)$. Let $\overline{\text { tr }}$ denote the composition $\mathscr{P}_{E,-1} \stackrel{\text { tr }}{\longrightarrow} \omega \rightarrow \mathscr{H}, \mathscr{K}:=\operatorname{ker} \overline{\operatorname{tr}}$. Put $\hat{\mathscr{D}}_{E}=\mathscr{P}_{-1} / \mathscr{K}$ so we have the exact sequence

$$
0 \rightarrow \mathscr{K} \stackrel{\overline{\mathrm{r}}^{-1}}{\longrightarrow} \widehat{\mathscr{D}}_{E} \stackrel{\delta}{\longrightarrow} \mathscr{D}_{E} \rightarrow 0 .
$$

For $a, b \in \hat{\mathscr{D}}_{E}$ put $[a, b]_{V}:=\operatorname{Lie}(\delta a)(\tilde{b}) \bmod \mathscr{K} \in \hat{\mathscr{D}}_{E}$, where $\tilde{b} \in \mathscr{P}_{E}, \tilde{b} \bmod \mathscr{K}=b$, and 2.1.1.3 implies the following statement

2.2.2. Lemma. $[\cdot, \cdot]_{V}$ is a well defined Lie bracket on $\hat{\mathscr{D}}_{E}$; so $\hat{\mathscr{D}}_{E}$ becomes a central extension of $\mathscr{D}_{E}^{\mathrm{Lie}}$ by $\mathscr{H}$.

Now assume that $X$ is compact. Then $H^{1}(X, \mathscr{H})=H_{\mathrm{DR}}^{2}(X)=\mathbb{C}$, hence the boundary map in 2.2.1 defines the linear function $\operatorname{tr}: H^{0}\left(X, \mathscr{D}_{E}\right) \rightarrow H^{1}(X, \mathscr{H})=\mathbb{C}$.

2.2.3. Lemma. $\operatorname{tr}=\operatorname{tr}_{H(X, E)}$, i.e. $\operatorname{tr}(d)$ is the alternating sum of traces of d's action on $H^{*}(\mathrm{X}, \mathrm{E})$ for a differential operator $\mathrm{d}$.

Proof. Consider the exact sequence $0 \rightarrow E \otimes E^{0} \rightarrow E \otimes E^{0}(\infty \Delta) \rightarrow \mathscr{D}_{E} \rightarrow 0$ of sheaves on $X \times X$. We have $H^{+1}\left(X \times X, \quad E \otimes E^{0}\right)=H^{*}(X, E) \otimes H^{1-+^{+} \cdot}\left(X, E^{0}\right)$ $=H^{*}(X, E) \otimes H^{-*}(X, E)^{*}=\operatorname{End}\left(H^{*}(\mathrm{X}, \mathrm{E})\right)$ by Kunneth and Serre's duality. The map $\operatorname{End}^{0}\left(H^{\cdot}(X, E)\right)=H^{1}\left(X \times X, E \otimes E^{0}\right) \stackrel{\text { tr } \lambda^{*}}{\longrightarrow} H^{1}\left(X, \Omega^{1}\right)=\mathbb{C}$ coincides with the trace of the action on $H^{\prime}(X, E)$. The boundary map $H^{\prime}\left(X, \mathscr{D}_{E}\right) \rightarrow H^{+1}(X \times X$, $\left.E \otimes E^{0}\right)=$ End $^{\cdot}\left(H^{\cdot}(X, E)\right)$ coincides with the map induced by the action of $\mathscr{D}_{E}$ on $E$. These two easy facts imply the lemma.

Remark. This lemma implies the Riemann-Roch formula for $\chi(X, E)$ : one has $\chi(X, E)=\operatorname{tr}_{H(E)} \mathrm{id}_{E}$, and this may be computed in Čech cohomology using gauge change formulas for ${ }^{\text {tr }} \mathscr{A}^{-1}$ from 2.1.2 (this is a one-dimensional version of ToledoTong's proof of Riemann-Roch, [24]).

\subsection{Atiyah Algebra of Determinant Bundle}

The principal point of this section is the following

2.3.1. Theorem. There is a canonical isomorphism between $\mathcal{O}_{S}$-Atiyah algebras,

$$
I_{E}: \pi\left({ }^{\mathrm{tr}} \mathscr{A}_{E}\right) \stackrel{\sim}{\rightarrow} \mathscr{A}_{\lambda_{E}},
$$

where $\lambda_{E}:=\operatorname{det} R \pi_{*} E$ is the line bundle on $S$ defined by Grothendieck, see $[14,11,7]$.

Remark. In [14] $\operatorname{det} R \pi_{*} E$ was defined as a graded line bundle; we omit the grading since the sign problems are irrelevant for us.

The proof of 2.3.1 will occupy the rest of the subsection.

2.3.2. We will start with the list of compatibilities that, in fact, determine $I_{E}$.

(i) Let $0 \rightarrow E_{1} \rightarrow E \rightarrow E_{2} \rightarrow 0$ be a short exact sequence of vectors bundles. Then

$$
I_{E}=I_{E_{1}}+I_{E_{2}},
$$

where we identified $\mathscr{A}_{\lambda_{\mathrm{E}}}$ with $\mathscr{A}_{\lambda_{E_{1}}}+\mathscr{A}_{\lambda_{E_{2}}}$ by means of the canonical isomorphism $\lambda_{E}=\lambda_{E_{1}} \otimes \lambda_{E_{2}}$ and the identification $\pi\left({ }^{\mathrm{tr}} \cdot \mathscr{A}_{E}^{*}\right)=\pi\left({ }^{\mathrm{tr}} \cdot \mathscr{A}_{\dot{E}_{1}}\right)+\pi\left({ }^{\mathrm{tr}} \cdot \mathscr{A}_{E_{2}}\right)$ is defined as

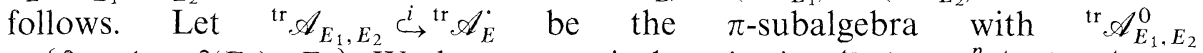
$=\left\{\partial \in \mathscr{A}_{E, \pi}: \partial\left(E_{1}\right) \subset E_{1}\right\}$. We have canonical projection ${ }^{\mathrm{tr}} \mathscr{A}_{E_{1}, E_{2}} \stackrel{p}{\rightarrow}{ }^{\mathrm{t}} \mathscr{A}_{E_{1}} \times{ }^{\mathrm{t}} \mathscr{\mathscr { A }}_{E_{2}}$ : 
here $p^{0}$ is the induced action on $E_{1}, E_{2}$, and $p^{-1}$ comes from the identification of $\mathscr{P}_{E_{1}} \times \mathscr{P}_{E_{2}}$ with the subquotient of $\mathscr{P}_{E}$ [namely, $\mathscr{P}_{E_{1}} \times \mathscr{P}_{E_{2}}$ is the quotient of $L \otimes \mathscr{P}$ $C\left(E^{*} \otimes E\right) \otimes \mathscr{P}$, where $L:=\operatorname{Ker}\left(E^{*} \otimes E \rightarrow E_{1}^{*} \bowtie E_{2}\right)$. The diagram of $\pi$-algebras

$$
{ }^{\operatorname{tr}} \mathscr{A}_{E} \stackrel{i}{ }{ }^{\operatorname{tr}} \mathscr{A}_{E_{1}, E_{2}} \stackrel{p}{\longrightarrow}{ }^{\operatorname{tr}} \mathscr{A}_{E_{1}} \times{ }^{\mathrm{tr}} \mathscr{A}_{E_{2}}
$$

gives the desired isomorphism

$$
\pi(p) \pi(i)^{-1}: \pi\left({ }^{\mathrm{tr}} \mathscr{A}_{E}\right) \simeq \pi\left({ }^{\mathrm{tr}} \mathscr{A}_{E_{1}}\right)+\pi\left({ }^{\mathrm{tr}} \mathscr{A}_{E_{2}}\right) .
$$

(ii) Let $D \stackrel{i}{\hookrightarrow} X$ be a divisor étale over $S$. Consider the exact sequence $\left.0 \rightarrow E(-D) \rightarrow E \rightarrow i_{*} E\right|_{D} \rightarrow 0 ; \quad$ put $E_{D}:=\left.\pi\right|_{D^{*}}\left(\left.E\right|_{D}\right)$. The isomorphism $\lambda_{E}=\lambda_{E(-D)} \otimes \operatorname{det} E_{D}$ identifies $\mathscr{A}_{\lambda_{E}}$ with $\mathscr{A}_{\lambda_{E(-D)}}+\mathscr{A}_{\operatorname{det} E_{D}}$. In a moment we will define the isomorphism $\pi\left({ }^{\mathrm{tr}} \mathscr{A}_{E}\right) \simeq \pi\left({ }^{\mathrm{tr}} \mathscr{A}_{E(-D)}\right)+\mathscr{A}_{\operatorname{det} E_{D}}$, and our second compatibility claims that

$$
I_{E}=I_{E(-D)}+{ }^{\mathrm{id}} \mathscr{A}_{\operatorname{det} E_{D}}
$$

So let ${ }^{\mathrm{tr}} \mathscr{A}_{E, D} \subset{ }^{\mathrm{tr}} \mathscr{A}_{E}$ be a $\pi$-subalgebra such that ${ }^{\mathrm{tr}} \mathscr{A}_{E, D}^{0}=\left\{\partial \in \mathscr{A}_{E, \pi}: \varepsilon(\partial)\right.$ is tangent to $D\}$; one has projections $p:{ }^{\text {tr }} \mathscr{A}_{E, D}^{0} \rightarrow^{\text {tr }} \mathscr{A}_{E(-D)}^{0}, q:{ }^{\mathrm{t}} \mathscr{A}_{E, D}^{0} \rightarrow i_{D^{*}} \mathscr{A}_{\left.E\right|_{D}}$. Let $\mathscr{L}^{\cdot} C^{\operatorname{tr}} \mathscr{A}_{E, D}$ be the dg-ideal with $\mathscr{L}^{i}={ }^{\text {tr }} \mathscr{A}_{E, D}^{i}$ for $i \neq-1, \mathscr{L}^{-1}=\operatorname{Ker} q d^{-1}$. Then $p, q$ extend to dg-maps $p: \mathscr{L}^{\cdot} \rightarrow{ }^{\mathrm{tr}} \mathscr{A}_{E(-D)}, q: \mathscr{L}^{*} \rightarrow i_{D *} \mathscr{A}_{E}\left(\left.p\right|_{X \backslash D}\right.$ is the identity map). Then $R^{0} \pi_{*}(p+q): R^{0} \pi_{*}\left(\mathscr{L}^{*}\right) \rightarrow\left({ }^{\mathrm{tr}} \mathscr{A}_{E(-D)}^{\cdot}\right) \times\left._{\mathscr{T}_{S}} \pi\right|_{D}\left(\mathscr{A}_{\left.E\right|_{D}}\right)$ is an isomorphism, and the corresponding map $\left.\alpha: \pi{ }^{\text {tr }} \mathscr{A}_{E(-D)}\right) \times\left.{ }_{\mathscr{T} S} \pi\right|_{D}\left(\mathscr{A}_{\left.E\right|_{D}}\right) \rightarrow R^{0} \pi_{*}\left(\mathscr{L}^{*}\right)$ gives the desired isomorphism $\left.\quad \pi\left({ }^{\mathrm{tr}} \mathscr{A}_{E}\right) \stackrel{\sim}{\rightarrow} \pi{ }^{\mathrm{tr}} \mathscr{A}_{E(-D)}\right)+\mathscr{A}_{\operatorname{det} E_{D}} \quad\left[\right.$ since $\quad \mathscr{A}_{\operatorname{det} E_{D}}=\left.\operatorname{tr} \pi\right|_{D}\left(\mathscr{A}_{\left.E\right|_{D}}\right) \quad$ and $\alpha$ on $\left.\pi\right|_{D^{*}}\left(\right.$ End $\left.\left.E\right|_{D}\right)$ coincides with tr by 2.2.2].

(iii) Assume that $R^{0} \pi_{*} E=R^{1} \pi_{*} E=0$. Then $\operatorname{det} R \pi_{*} E=\mathcal{O}_{S}$, hence $I_{E}$ is just the trivialisation of $\pi\left({ }^{\mathrm{tr}} \mathscr{A}_{E}\right)$, i.e. the integrable connection $\left.\nabla: \mathscr{T}_{S} \rightarrow \pi{ }^{\mathrm{tr}} \mathscr{A}_{E}\right)$. Now recall that ${ }^{\mathrm{tr}} \mathscr{A}_{E}^{-1}$ is a quotient of a subsheaf $\mathscr{B}_{E} \subset E \otimes E^{0}(2 \Delta)$ [here $\mathscr{B}_{E}$ consists of sections that are mapped into $\mathscr{A}_{E / S} \subset \mathscr{D}_{E, 2}$ under the "pole at $\Delta$ " map: $\left.E \bowtie E^{0}(2 \Delta) \stackrel{\delta}{\rightarrow} \mathscr{D}_{E, 1}\right]$, so we get a morphism of complexes $\beta: \operatorname{Cone}\left(\mathscr{B}_{E} \stackrel{\delta}{\rightarrow} \mathscr{A}_{E, \pi}\right) \rightarrow{ }^{\mathrm{tr}} \mathscr{A}_{E}$. The exact triangle

$$
E 凶 E^{0}[1] \rightarrow \operatorname{Cone}\left(\mathscr{B}_{E} \rightarrow \mathscr{A}_{E, \pi}\right) \rightarrow \pi^{-1} \mathscr{T}_{S}
$$

shows that $R^{0} \pi_{*}$ Cone $=\mathscr{T}_{S}$ in our case $\left[\right.$ since $R \pi_{*}\left(E 区 E^{0}[1]\right)=$ End $R \pi_{*} E=0$ ], hence $R^{0} \pi_{*} \beta$ is just the section $\mathscr{T}_{S} \rightarrow \pi\left({ }^{\operatorname{tr}} \mathscr{A}_{E}\right)$ and (iii) claims that

$$
\nabla=R^{0} \pi_{*} \beta \text {. }
$$

(iv) Compatibility with base change. Both assignments $E \sim \rightarrow \lambda_{E}, E \sim \rightarrow{ }^{\text {tr }} \mathscr{A}_{E}$ are compatible with base change (change of $S$ ), hence so should $I_{E}$.

Now we can give a precise formulation of 2.3.1:

Theorem. There exists a unique system of isomorphisms $I_{E}$ such that compatibilities (i)-(iv) above hold.

Note that the uniqueness is clear: by (iv) the problem is local in $S$, so by (i) we may assume that $E$ is a line bundle, and, using (ii), we may reduce the arguments to the situation described in (iii). It remains to construct $I_{E}$.

2.3.3. Construction of $I_{E}$ in case $R^{1} \pi_{*} E=0$. Here $\pi_{*} E$ is a vector bundle on $S$ and $\lambda_{E}=\operatorname{det} \pi_{*} E$ hence $\mathscr{A}_{\lambda_{E}}=\operatorname{tr} \mathscr{A}_{\pi_{*} E}$. Consider the exact triangle 2.3.2.1; it gives the short exact sequence

$$
0 \rightarrow \text { End } \pi_{*} E \rightarrow R^{0}(\pi \times \pi)_{*} \text { Cone } \rightarrow \mathscr{T}_{S} \rightarrow 0
$$


since $R(\pi \times \pi)_{*}\left(E \otimes E^{0}\right)=R \pi_{*} E \otimes\left(R \pi_{*} E\right)^{*}=$ End $\pi_{*} E$ by Kunneth and Serre's duality. We will construct the natural isomorphism $\tilde{I}_{E}: \mathscr{A}_{\pi_{*} E} \stackrel{\sim}{\rightarrow} R^{0}(\pi \times \pi)_{*}$ (Cone), compatible with 2.3.1. Since $\operatorname{tr}:$ End $\pi_{*} E \rightarrow \mathcal{O}_{S}$ coincides with $\operatorname{Tr} \cdot R(\pi \times \pi)_{*}(\varphi)$, where

$$
\begin{gathered}
\varphi: E \otimes E^{0} \stackrel{\Delta^{*}}{\longrightarrow} E \otimes E^{0} \stackrel{\mathrm{tr}}{\longrightarrow} \omega, \\
\operatorname{Tr}: R^{1} \pi_{*} \omega \rightarrow \mathcal{O}_{S},
\end{gathered}
$$

and ${ }^{\operatorname{tr}} \mathscr{A}_{E}^{-1}=\mathscr{B}_{E} / \operatorname{Ker} \varphi$, the morphism $\beta:$ Cone $\rightarrow{ }^{\operatorname{tr}} \mathscr{A}_{E}$ and $\tilde{I}_{E}$ define the desired $I_{E}: \operatorname{tr} \mathscr{A}_{\pi_{*} E} \stackrel{\sim}{\rightarrow} \pi\left({ }^{\mathrm{tr}} \mathscr{A}_{E}^{\dot{E}}\right)$.

To define $\tilde{I}_{E}$ one proceeds as follows. The problem is local in $S$, so let us choose some relative divisor $T \subset X, T$ is finite over $S$; put $T^{(2)}:=X \times{ }_{S} T \subset X \times{ }_{S} X$. Then, since $R^{1} \pi_{*} E=0$, the sheaves $\mathscr{A}_{E, \pi}(\infty T)\left(:=\right.$ sheaf of sections of $\mathscr{A}_{E, \pi}$ that may have a singularity at $T), E \otimes E^{0}\left(\infty T^{(2)}\right)$ and $\mathscr{B}_{E}\left(\infty T^{(2)}\right)$ are acyclic with respect to projection on $S$. Hence $R^{0}(\pi \times \pi)_{*}$ Cone may be calculated using the Cousin resolution of Cone:

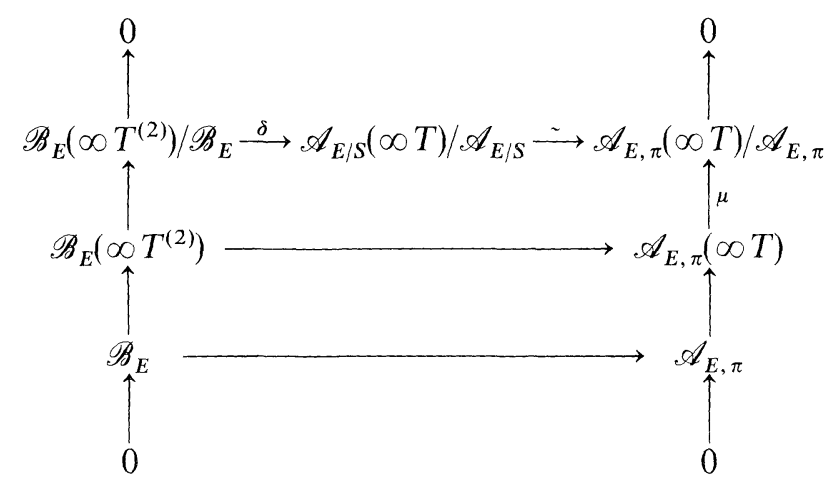

Namely,

$$
\begin{aligned}
& R^{0}(\pi \times \pi)_{*}(\text { Cone })=\left\{(\tau, b) \in \pi_{*} \mathscr{A}_{E, \pi}(\infty T)\right. \\
& \left.\times \pi_{*}\left(\mathscr{B}_{E}\left(\infty T^{(2)}\right) / \mathscr{B}_{E}\right): \mu(\tau)=\delta(b)\right\} / \text { Image of } \pi_{*} \mathscr{B}_{E}\left(\infty T^{(2)}\right) .
\end{aligned}
$$

Define the inverse to $\tilde{I}_{E}$ by the formula

$$
\tilde{I}_{E}^{-1}(\tau, b)(e)=\tau(e)+\operatorname{Res}_{T^{(2)}} b \cdot p_{2}^{*} e,
$$

where $e \in \pi_{*} E, b \cdot p_{2}^{*} e \in(E 区 \omega)\left(2 \Delta+\infty T^{(2)}\right) / E \bigotimes \omega(2 \Delta)$, and the residue is taken along the second variable. Note that the condition on $(\tau, b)$ implies that singularities at $T$ of the two terms in the right-hand side of the formula cancel. It is easy to see that $\tilde{I}_{E}^{-1}$ so defined is an isomorphism (it induces the identity maps on the left and right terms of 2.3.3.1) that does not depend on the choice of $T$ (it is obvious for $T_{1} \subset T_{2}$, the general case reduces to this one).

Remark. Note that the Cousin resolution for ${ }^{\text {tr }} \mathscr{A}_{E}$ gives (for arbitrary $E$ !) the isomorphism

$$
\begin{aligned}
\pi\left({ }^{\mathrm{tr}} \mathscr{A}_{E}\right)= & \left\{(\tau, a) \in \pi_{*} \mathscr{A}_{E, \pi}(\infty T) \times \pi_{*}{ }^{\mathrm{tr}} \mathscr{A}_{E}^{-1}(\infty T) /{ }^{\mathrm{tr}} \mathscr{A}_{E}^{-1}:\right. \\
& \left.\mu(\tau)=\delta(a)\} / \text { Image of } \pi_{*}{ }^{\mathrm{tr}} \mathscr{A}_{E}^{-1}(\infty T)\right) .
\end{aligned}
$$


So to calculate $I_{E}^{-1}(\tau, a)$ one should choose $(\tau, b)$ in 2.3.3.3 so as $\beta(b)=a$, then $I_{E}^{-1}(\tau, a)=\operatorname{tr} \widetilde{I}_{E}^{-1}(\tau, b)$.

2.3.4. $I_{E}$ Commutes with Brackets. For $\alpha_{i} \in \pi\left({ }^{\text {tr }} \mathscr{A}_{E}\right), i=1,2$, in (2.3.3.5) choose the representatives $\left(\tau_{i}, a_{i}\right)$ of $\alpha_{i}$ with respect to divisors $T_{i}$ such that $T_{1} \cap T_{2}=\emptyset$ and the corresponding $\left(\tau_{i}, b_{i}\right)$ such that $\beta\left(b_{i}\right)=a_{i}$. Then $\left(\left[\tau_{1}, \tau_{2}\right],\left[\tau_{1}, a_{2}\right]-\left[\tau_{2}, a_{1}\right]\right)$ is $T_{1} \cup T_{2}$-representative of $\left[\alpha_{1}, \alpha_{2}\right]$. Since

$$
\begin{aligned}
& {\left[\tilde{I}_{E}^{-1}\left(\tau_{1}, b_{1}\right), \tilde{I}_{E}^{-1}\left(\tau_{2}, b_{2}\right)\right]} \\
& \quad=\tilde{I}_{E}^{-1}\left(\left[\tau_{1}, \tau_{2}\right], \tau_{1} b_{2}-b_{2} \tau_{1}+b_{1} \tau_{2}-\tau_{2} b_{1}+b_{1} * b_{2}-b_{2} * b_{1}\right)
\end{aligned}
$$

[here $\tau_{i} b_{j}, b_{j} \tau_{i}$ are taken with respect to the $A_{E, \pi}$-bimodule structure on $E \bowtie E^{0}$ and $*$ is a convolution, so $b_{1} * b_{2}$ is a section of $\mathscr{B}_{E}\left(\infty T_{2}^{(2)}\right) / \mathscr{B}_{E}$ perhaps with a pole at $\left.T_{1} \times T_{2}\right)$, then we have $I_{E}^{-1}\left[\alpha_{1}, \alpha_{2}\right]-\left[I_{E}^{-1} \alpha_{1}, I_{E}^{-1} \alpha_{2}\right]=\operatorname{Res}_{T_{2}}\left(\beta\left(b_{1} * b_{2}\right)\right)$ $-\operatorname{Res}_{T_{1}}\left(\beta\left(b_{2} * b_{1}\right)\right)$. These residues are traces of finite rank operators $A_{b_{1}, b_{2}} \in \operatorname{End}_{\mathscr{O}_{S}} \hat{E}_{T_{2}}, A_{b_{2}, b_{1}} \in \operatorname{End}_{\mathcal{O}_{S}} \hat{E}_{T_{1}}$ (where $\hat{E}_{T_{\imath}}$ is formal completion of $E$ along $T_{i}$ ) defined by the formula $A_{b_{i} b_{j}}\left(e_{j}\right)=\operatorname{Res}_{T_{j}}\left(b_{i} * b_{j} \cdot p_{2}^{*} e_{j}\right)$ (see 2.3.3.4). Clearly $A_{b_{i} b_{j}}$ $=A_{b_{i}} \cdot A_{b_{j}}$, where $A_{b_{i}} \in \operatorname{Hom}_{\mathcal{O}_{S}}\left(\hat{E}_{T_{i}}, \hat{E}_{T_{j}}\right)$ are finite rank operators defined by the formula $A_{b_{1}}\left(e_{i}\right)=$ the expansion of $\operatorname{Res}_{T_{i}}\left(b_{i} p_{i}^{*} e_{i}\right)$ at $T_{i}$. Hence $I_{E}^{-1}\left[\alpha_{1}, \alpha_{2}\right]$ $-\left[I_{E}^{-1} \alpha_{1}, I_{E}^{-1} \alpha_{2}\right]=\operatorname{Tr} A_{b_{1}} \cdot A_{b_{2}}-\operatorname{Tr} A_{b_{2}} \cdot A_{b_{1}}=0$.

2.3.5. Case of Arbitrary E. The above defined $I_{E}$ satisfies 2.3.2 (iii), (iv), and also 2.3 .2 (i), (ii) in cases $R^{1} \pi_{*} E_{1}=R^{1} \pi_{*} E_{2}^{i}=0, R^{1} \pi_{*} E(-D)=0$ respectively. Now for arbitrary $E$ choose $D$ as in 2.3.2 (ii) so as $R^{1} \pi_{*} E(D)=0$, and define $I_{E}$ by means of $I_{E(D)}$ via (2.3.2)(ii). By the previous remark this $I_{E}$ does not depend on $D$ and satisfies 2.3.2(i)-(iv). So we are done.

The rest of the section contains some complements that will not be of much use in what follows.

\subsection{Relation with Tate's Construction}

In this subsection we also assume for brevity that $S=$ point and $T \subset X(\mathbb{C})$ consists of a single point $x$. Let $t$ be a parameter at $x, t(x)=0, U$ be a formal neighbourhood of $x, \mathcal{O}_{U}=\mathbb{C}[[t]]$ and $\dot{U}=U \backslash T$ is a punctured neighbourhood of $x, \mathcal{O}_{U}=\mathbb{C}((t))$. Then $\operatorname{Res}_{x}: \mathscr{H}^{1}(\dot{U}) \stackrel{\sim}{\rightarrow}$, hence 2.2 .1 gives a central extension $\hat{\mathscr{D}}(\dot{U})$ of $\mathscr{D}(\dot{U})$ $=\mathscr{D}_{E} \otimes \mathcal{O}_{U}$ by $\mathbb{C}$. We will see that $\hat{\mathscr{D}}(\dot{U})$ is canonically isomorphic to the extension constructed by Tate [20] by means of the linear algebra.

Remark. This construction became famous since it was rediscovered by the Kyoto school in connection with soliton theory and representations $[10,19,12]$.

2.4.1. Recall Tate's construction. Put $F=E(\dot{U})$, so $F \approx \mathbb{C}((t))^{n} \supset E(U) \approx \mathbb{C}[[t]]^{n}$. We say that a $\mathbb{C}$-vector subspace $V \subset F$ is open iff $V \supset t^{N} E(U)$ and $V$ is bounded iff $V \subset t^{-N} E(U)$ for $N \gg 0$; a subspace both open and bounded will be called a lattice. Let $\mathscr{R} \subset \operatorname{End}_{\mathbb{C}} F$ be the subring of continuous operators $\left[A \in \mathscr{R}\right.$ if and only if $A^{-1}(V)$ is open for any open $V \subset F], I_{0}:=\{A \in \mathscr{R}: \operatorname{Im} A$ is bounded $\}, I_{1}:=\{A \in \mathscr{R}: \operatorname{Ker} A$ is open $\}, I_{01}:=I_{0} \cap I_{1}$; these are two-sided ideals in $\mathscr{R}$ such that $I_{0}+I_{1}=\mathscr{R}$. If $A \in I_{01}$, then $\operatorname{dim} \operatorname{Im} A^{2}<\infty$, so $\operatorname{tr} A$ is well defined and $\operatorname{tr}[a, b]=0$ for $a \in I_{0}, b \in I_{1}$. 
Put $Q:=I_{0} \oplus I_{1}$; we have the exact sequence

$$
0 \rightarrow I_{01} \stackrel{i}{\longrightarrow} Q \stackrel{\delta}{\longrightarrow} \mathscr{R} \rightarrow 0,
$$

$i(A)=(A,-A), \delta(A, B)=A+B$. Now $Q$ is an $\mathscr{R}$-bimodule in an obvious way, hence $\mathscr{R}^{\text {Lie }}$ acts on $Q$ by the formula $\operatorname{Lie}(r)(q)=r q-q r$. Put $\mathscr{K}=\operatorname{Ker} \operatorname{tr}: I_{01} \rightarrow \mathbb{C}$, and $\widehat{\mathscr{R}}:=Q / i(\mathscr{K})$, so that we have the exact sequence

$$
0 \rightarrow \mathbb{C} \stackrel{i \cdot \operatorname{tr}^{-1}}{\longrightarrow} \widehat{\mathscr{R}} \stackrel{\delta}{\longrightarrow} \mathscr{R} \rightarrow 0 .
$$

The formula $[a, b]:=\operatorname{Lie} \delta(a)(\tilde{b}), a, b \in \mathscr{R}, \tilde{b} \in Q, \tilde{b}=b \bmod i(\mathscr{K})$, defines the Lie algebra bracket on $\widehat{\mathscr{R}}$, therefore (2.4.1.2) is a central extension of $\mathscr{R}^{\text {Lie }}$ by $\mathbb{C}$. Note that (2.4.1.2) canonically splits over $I_{0}$ and $I_{1}$ (by the definition of $Q$ ); let $s_{0}: I_{0} \rightarrow \widehat{\mathscr{R}}$, $s_{1}: I_{1} \rightarrow \widehat{\mathscr{R}}$ be these sections. Then $s_{i}\left(I_{i}\right)$ are ideals, and $s_{0}(A)-s_{1}(A)=\operatorname{tr} A$ for $A \in I_{01}$.

2.4.2. To relate this to Virasoro algebra, consider the local $\mathscr{P}_{E}$ 's: $\mathscr{P}_{E,-1}(\dot{U}) \subset \mathscr{P}_{E}(\dot{U})$, $\mathscr{P}_{E,-1}(U)=E(\dot{U}) \otimes \mathbb{C}\left[\left[t_{1}, t_{2}\right]\right] \otimes E^{0}(\dot{U}), \mathscr{P}_{E}(\dot{U})$ is the localisation of $\mathscr{P}_{E,-1}(U)$ by $\left(t_{1}-t_{2}\right)^{-1}$. We have the exact sequence of $\mathscr{D}_{E}(\dot{U})$-bimodules,

$$
0 \rightarrow \mathscr{P}_{E,-1}(\dot{U}) \rightarrow \mathscr{P}_{E}(\dot{U}) \stackrel{\delta}{\longrightarrow} \mathscr{D}_{E}(\dot{U}) \rightarrow 0 \text {. }
$$

Let R: $\mathscr{D}_{E}(\dot{U}) \rightarrow \mathscr{R}$ denote the action of $\mathscr{D}_{E}(\dot{U})$ on $E(\dot{U})$; this is an embedding of $\mathbb{C}$-algebras. For $\psi=\psi\left(t_{1}, t_{2}\right) d t_{2} \in \mathscr{P}_{E}(\dot{U})$ define $\operatorname{Res}_{0}(\psi), \operatorname{Res}_{\Delta}(\psi) \in \mathscr{R}$ by the formulas

$$
\begin{aligned}
& \left(\operatorname{Res}_{0}(\psi)(f)\right)(t)=\operatorname{Res}_{t_{2}=0} \psi\left(t, t_{2}\right) f\left(t_{2}\right) d t_{2}, \\
& \left(\operatorname{Res}_{\Delta}(\psi)(f)\right)(t)=\operatorname{Res}_{t_{2}=t} \psi\left(t, t_{2}\right) f\left(t_{2}\right) d t_{2} .
\end{aligned}
$$

One has

(i) $\operatorname{Res}_{\Delta}=\mathrm{R} \cdot \delta$, and $\operatorname{Res}_{0}, \operatorname{Res}_{A}: \mathscr{P}_{E}(\dot{U}) \rightarrow \mathscr{R}$ are $R$-morphisms of $\mathscr{D}_{E}(\dot{U})$ bimodules.

(ii) $\operatorname{Res}_{0}\left(\mathscr{P}_{E}\right) \subset I_{1},\left(\operatorname{Res}_{0}+\operatorname{Res}_{\Delta}\right)\left(\mathscr{P}_{E}\right) \subset I_{0}$, hence we may define an $R$-morphism of bimodules

$$
\operatorname{Res}: \mathscr{P}_{E}(\dot{U}) \rightarrow Q, \operatorname{Res}(\psi)=\left(\operatorname{Res}_{0}+\operatorname{Res}_{\Delta},-\operatorname{Res}_{0}\right) .
$$

(iii) $\mathrm{R} \delta=\delta$ Res, so (Res, R) defines the embedding (2.4.2.1) $\hookrightarrow$ (2.4.1.1).

(iv) $\left.\operatorname{Res}\right|_{\mathscr{P}_{E,-1}(U)}: \mathscr{P}_{E,-1}(\dot{U}) \rightarrow I_{01}$ is an isomorphism compatible with traces from 2.2.1 and 2.4.1.

So Res modulo Kertr identifies $\hat{\mathscr{D}}_{E}(\dot{U})$ with a subalgebra of $\hat{\mathscr{R}}$.

\subsection{Shifted Virasoro Algebras}

For an integer $i$ consider the sheaf $\mathscr{P}_{E}(i):=\omega^{\otimes i} \otimes_{\mathcal{O}_{\mathrm{X}}} \mathscr{P}_{E} \otimes_{\mathscr{C}_{\mathrm{X}}} \omega^{\otimes i}$ filtered by $\mathscr{P}_{E^{(i)} a}:=\omega^{\otimes i} \mathscr{P}_{E, a-2 i} \omega^{\otimes i}$. Clearly, $\mathscr{A}_{E, \pi}$ [being the algebra of symmetries of $\left.(E, \pi)\right]$ acts naturally on $\mathscr{P}_{E}(i)$, and $\mathrm{Gr}_{a} \mathscr{P}_{E}(i)=\omega^{\otimes-a} \cdot$ End $E$. Put

$$
\mathscr{A}_{E / S}(i):=\left\{f \in \mathscr{P}_{E}(i)_{1} / \mathscr{P}_{E}(i)_{-1}: \varepsilon(f):=f \bmod \mathscr{P}_{E}(i)_{0} \in \omega^{-1} \mathrm{Id}_{E}\right\},
$$


hence we have a short exact sequence $0 \rightarrow \operatorname{End} E \rightarrow \mathscr{A}_{E / S}(i) \stackrel{\varepsilon}{\rightarrow} \mathscr{T}_{X / S} \rightarrow 0$. Put

$$
\hat{\mathscr{A}}_{E / S}(i):=\left\{f \in \mathscr{P}_{E}(i)_{1} / \mathscr{P}_{E}(i)_{-2}: \delta(f):=f \bmod \mathscr{P}_{E}(i)_{-1} \in \mathscr{A}_{E / S}(i)\right\} / \operatorname{Kertr},
$$

where $\operatorname{tr}$ is a composition $\mathscr{A}_{E / S}(i)_{-1} / \mathscr{A}_{E / S}(i)_{-2}=\omega \cdot$ End $E \stackrel{\operatorname{tr}}{\rightarrow} \omega \rightarrow \omega / d \mathcal{O}=\mathscr{H}$. So $\hat{\mathscr{A}}_{E / S}(i)$ is an extension of $\mathscr{A}_{E / S}(i)$ by $\mathscr{H}$. Given a gauge $(t, I)$ for $(X, E)$ (see 2.1) we have compatible isomorphisms $s_{(t, I)}: \mathscr{T}_{X / S} \oplus \operatorname{Mat}_{n}\left(\mathcal{O}_{X}\right) \stackrel{\sim}{\rightarrow} \mathscr{A}_{E / S}(i), \mathscr{T}_{X / S} \oplus \operatorname{Mat}_{n}\left(\mathcal{O}_{X}\right)$ $\oplus \mathscr{H} \stackrel{\sim}{\rightarrow} \hat{\mathscr{A}}_{E / S}(i)$ defined by the formula

$$
s_{(t, I)}\left(\tau(t) \partial_{t}, B(t), v(t) d t\right)=(\tau(t), B(t), v(t))_{(t, I)}^{(i)}
$$

same for $\mathscr{A}_{E / S}(i)$.

$$
=I \otimes I\left[\frac{\tau\left(t_{1}\right)}{\left(t_{2}-t_{1}\right)^{2+2 i}}+\frac{B\left(t_{1}\right)}{\left(t_{2}-t_{1}\right)^{1+2 i}}+\frac{v\left(t_{1}\right)}{\left(t_{2}-t_{1}\right)^{2 i}}\right] d t_{1}^{i} d t_{2}^{i+1},
$$

Lemma. a) We have canonical isomorphisms $\mathscr{A}_{E / S}(i) \cong \mathscr{A}_{E / S}(0)=\mathscr{A}_{E / S}$, $(\tau, B)_{(t, I)}^{(i)} \mapsto(\tau, B)_{(t, I)}$, that commute with the $\mathscr{A}_{E / S^{-a c t i o n}}$.

b) Define the bracket on $\hat{\mathscr{A}}_{E / S}(i)$ by the formula $[\alpha, \beta]=\operatorname{Lie}_{\delta(\alpha)}(\beta)$. This bracket is skew-symmetric, hence defines a Lie algebra structure on $\hat{\mathscr{A}}_{E / S}(i) ;$ so $\hat{\mathscr{A}}_{E / S}(i)$ is a central extension of $\mathscr{A}_{E / S}(i)$ by $\mathscr{H}$. The explicit formula for $[\cdot, \cdot]$ :

$$
\begin{aligned}
& {\left[\left(\tau_{1}, B_{1}, v_{1}\right)_{(t, I)}^{(i)},\left(\tau_{2}, B_{2}, v_{2}\right)_{(t, I)}^{(i)}\right]=\left(\tau_{1} \tau_{2}^{\prime}-\tau_{2} \tau_{1}^{\prime}, \tau_{1} B_{2}^{\prime}-\tau_{2} B_{1}^{\prime}\right.} \\
& \left.\quad+\left[B_{1}, B_{2}\right], \operatorname{Tr}\left(\frac{i+1}{12}\left(\tau_{1}^{\prime \prime \prime} \tau_{2}-\tau_{1} \tau_{2}^{\prime \prime \prime}\right)+\frac{1}{2}\left(\tau_{1}^{\prime \prime} B_{2}-\tau_{2}^{\prime \prime} B_{1}\right)-B_{2} B_{1}\right)\right)_{(t, I)}^{(i)} .
\end{aligned}
$$

Lemma follows from the direct calculation cf. [4].

Note also that we may take $i$ to be half-integer if the $\theta$-characteristic $\omega^{1 / 2}$ is chosen. The formula for the bracket above means that we have a canonical isomorphism $\hat{\mathscr{A}}_{E / S}(i) \cong \hat{\mathscr{A}}_{E / S}+i V,(\tau, B, v)_{(t, I)}^{(i)} \mapsto(\tau, B, v)_{(t, I)}+(\tau, 0,0)_{t}$. Here $V \subset \hat{\mathscr{A}}_{0_{X}}$ is Lie subalgebra of elements that project to $\mathscr{T}_{X / S} \subset \mathscr{A}_{\mathcal{O}_{X}}$ (so $V$ is a central extension of $\mathscr{T}_{X / S}$ by $\mathscr{H}$, and the sum of extensions is defined similarly to $\pi$-algebras case). Hence the corresponding $\mathcal{O}_{S}$-Atiyah algebra $R^{0} \pi_{*}\left(\hat{\mathscr{A}}_{E / S}(i) \rightarrow \mathscr{A}_{E, \pi}\right)$ coincides with $\pi\left({ }^{\mathrm{tr}} \mathscr{A}_{E}\right)+i \pi\left({ }^{\mathrm{tr}} \mathscr{A}_{\mathcal{O}_{X}}\right)=\mathscr{A}_{\lambda_{E} \otimes \lambda_{\mathbb{C}_{x}}^{\otimes i}}$.

\subsection{Modular Families}

Let $\pi: X \rightarrow S, E$ be a (locally) universal family of genus $g$ curves $(g>1)$ and vector bundles of rank $n$. This means that the Kodaira-Spencer map KS: $\mathscr{T}_{S} \rightarrow R^{1} \pi_{*} \mathscr{A}_{E / S}$ is isomorphism and $R^{0} \pi_{*} \mathscr{A}_{E / S}=R^{0} \pi_{*}$ End $E=\mathcal{O}_{S} \cdot \mathrm{id}_{E}$. Assume that $\chi\left(X_{s}, E_{s}\right)=0$, i.e. $\operatorname{deg} E=n(g-1)$. Then, according to 2.2 .3 , the exact sequence $0 \rightarrow \mathscr{H} \rightarrow \hat{\mathscr{A}}_{E / S}$ $\rightarrow \mathscr{A}_{E / S} \rightarrow 0$ gives the exact sequence of $R^{1} \pi_{*}$ 's: $0 \rightarrow \mathcal{O}_{S} \rightarrow R^{1} \pi_{*} \mathscr{A}_{E / S} \rightarrow \mathscr{T}_{S} \rightarrow 0$, here we identified $R^{1} \pi_{*} \mathscr{H}, R^{1} \pi_{*} \mathscr{A}_{E / S}$ with $\mathcal{O}_{S}, \mathscr{T}_{S}$ by means of Tr and KS. So the canonical map ${ }^{\operatorname{tr}} \mathscr{A}_{E} \rightarrow \sigma \leqq-1{ }^{\operatorname{tr}} \mathscr{A}_{E}=\operatorname{Cone}\left({ }^{\mathrm{tr}} \mathscr{A}_{E}^{-2} \rightarrow{ }^{\mathrm{tr}} \mathscr{A}_{E}^{-1}\right)[1] \rightarrow \hat{\mathscr{A}}_{E / S}$ defines the isomorphism $\hat{\mathrm{KS}}: \mathscr{A}_{\lambda_{E}} \stackrel{\sim}{\rightarrow} R^{0} \pi_{*}{ }^{\mathrm{tr}} \mathscr{A}_{E} \rightarrow R^{1} \pi_{*} \mathscr{A}_{E / S}$ of $\mathscr{O}_{S}$-modules that extends KS.

The Dual Statement. Put $Q_{E i}:=\operatorname{Ker}\left(\mathscr{P}_{E i} \rightarrow \mathscr{P}_{E i} / \mathscr{P}_{E i-1}=\omega^{-i+1}\right.$ End $\left.E \stackrel{\mathrm{tr}}{\longrightarrow} \omega^{-i+1}\right)$ $C \mathscr{P}_{i}, \overline{\mathscr{P}}_{E a b}=\mathscr{P}_{E a} / Q_{E b+1}$, so $\mathscr{P}_{E a b}$ is extension of $\mathscr{P}_{a, b+1}$ by $\omega^{-b}$. The Serre duality together with ${ }^{t}: \mathscr{P}_{E} \stackrel{\sim}{\rightarrow} \mathscr{P}_{E^{0}}$ identifies the dual to $R^{1} \pi_{*} \mathscr{P}_{E a b}$ with $\pi_{*} \mathscr{P}_{E 1-b, 1-a}$. Hence 
the dual to KS identifies $\Omega_{S}^{1}$ with $\pi_{*} \overline{\mathscr{P}}_{E 0,-2}$ and the dual to $\hat{\mathrm{KS}}$ is $\mathrm{C}\left(\lambda_{E}\right)\left(:=\right.$ the $\Omega_{S^{-}}^{1}$ torsor of connections on $\left.\lambda_{E}\right) \simeq\left\{\varphi \in \pi_{*} \overline{\mathscr{P}}_{E 1,-2}: \varphi \bmod \pi_{*} \mathscr{P}_{E, 0}=\mathrm{id}_{E}\right\}$. Using the shifted algebras we may identify $\Omega_{S}^{1}$ with $\pi_{*}\left(\omega^{2} \overline{\mathscr{P}}_{E 2 i, 2 i-2} \omega^{i}\right)$ and $C\left(\lambda_{E} \lambda_{\mathscr{O}_{\mathrm{X}}}^{\otimes i}\right)$ with $\left\{\varphi \in \omega^{i} \mathscr{P}_{E 2 i, 2 i-2} \omega^{i}: \varphi \bmod \pi_{*} \omega^{i} \mathscr{P}_{E 2 i, 2 i-2} \omega^{i}=\mathrm{id}_{E}\right\}$. In particular, if $i=1$, then

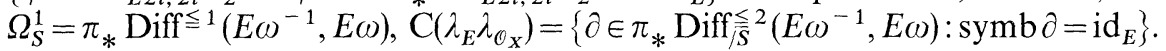

Example. Let $n=1$, so $S$ is moduli of genus $g$ curves with line bundle of degree $g-1$. Denote by $\mathscr{M}^{\vee}$ the moduli of pairs $\left(X, \omega^{1 / 2}\right), X$ is a curve, $\omega^{1 / 2}$ is $\theta$-characteristics. We have the obvious embedding $j: \mathscr{M}^{\vee} \hookrightarrow S$; put $X^{\vee}=\left.X\right|_{\mathscr{M}^{\vee}}$. Recall that $\hat{\mathscr{A}}_{\omega^{1 / 2} / \mathcal{M}^{\vee}}$ is the subquotient of $\mathscr{P}_{\omega^{1 / 2}} ;$ since $\omega^{1 / 20}=\omega^{1 / 2}, \mathscr{P}_{\omega^{1 / 2}}$ carries the involution ${ }^{t}$; let $\hat{\mathscr{A}}_{1 / 2} \stackrel{i}{\hookrightarrow} \hat{\mathscr{A}}_{\omega^{1 / 2} / \mathcal{M}^{\mathrm{v}}}$ be invariants of ${ }^{t}$ (cf. 3.1): this is extension of $\mathscr{T}_{X^{v} / \mathscr{M}^{v}}$ by $\mathscr{H}$. The same reasons as above show that $R^{1} \pi_{*} \hat{\mathscr{A}}_{1 / 2}=\mathscr{A}_{\lambda \omega^{1 / 2}}$, and $i$ induces the usual embedding $\mathscr{A}_{\lambda \omega^{1 / 2}} \hookrightarrow j^{*} \mathscr{A}_{\lambda_{E}}$.

One may translate this to 1 -shifted language. We have $\Omega_{\mathscr{M}}^{1}=\pi_{*} \omega^{2}$ and

$$
\mathrm{C}\left(\lambda_{\omega^{1 / 2}} \lambda_{\Theta_{X}}\right)=\left\{\partial \in \pi_{*} \operatorname{Diff}_{\bar{X} \vee / \mathcal{M}}^{\leq 2}\left(\omega^{-1 / 2}, \omega^{3 / 2}\right): \operatorname{symb} \partial=1,{ }^{t} \partial=\partial\right\} .
$$

Here ${ }^{t}$ is the conjugation of the differential operator [note that $\left(\omega^{3 / 2}\right)^{0}=\omega^{-1 / 2}$ ]. In 3.1 we will see that $\lambda_{\omega^{j}}=\lambda_{\mathscr{O}_{x}}, c_{j}=6 j^{2}-6 j+1$. Hence

$$
\mathrm{C}\left(\lambda_{\omega^{j}}\right)=\left\{\partial \in \pi_{*} \operatorname{Diff}_{\overline{\bar{X}} \vee / \mathcal{M}}^{\leq 2}\left(\omega^{-1 / 2}, \omega^{3 / 2}\right): \operatorname{symb} \partial=2 c_{j},{ }^{t} \partial=\partial\right\} .
$$

This fact is closely related to Tjurin's results [21]; cf. also [4].

\subsection{Problem}

It would be very interesting to "integrate" 2.3 . In a sense Deligne's Riemann-Roch (RR) theorem [11] performs implicitly this, but we would like to have a direct construction as well as a local construction of the RR isomorphism itself. More precisely, the "characteristic classes" in the right-hand side of Deligne's RR, say $\mathrm{IC}_{2}$, may be constructed as follows. Consider the central extension $\mathrm{GL}_{n}$ of $\mathrm{GL}_{n}$ by $\mathscr{K}_{2, X}$ that corresponds to $\mathrm{c}_{2}$. Let $E$ be a rank $n$ vector bundle, and $\mathscr{E}=\operatorname{Isom}\left(\mathcal{O}^{n}, E\right)$ its GL-torsor. One has a presheaf $\mathscr{C}(E)$ defined by formula $\mathscr{C}(E)(U)=\left\{\hat{G L}_{n^{-}}\right.$ structures on $\left.\left.E\right|_{U}\right\}=\left\{\left(\hat{\mathscr{E}}_{U}, i\right) ; \hat{\mathscr{E}}_{U}\right.$ is $\widehat{G L}_{n}$-torsor on $U, i: \widehat{\mathscr{E}}_{/} \mathscr{K}_{2} \stackrel{\sim}{\rightarrow} \mathscr{E}$, up to isomorphism $\}$. Let $\mathrm{IC}_{2}(E)$ be the sheaf that corresponds to the presheaf $\pi_{*} \mathscr{C}(E)$. Since $\pi$ is proper of relative dimension $1, \mathrm{IC}_{2}(E)$ is $R^{1} \pi_{*} \mathscr{K}_{2}$-torsor. The trace map $\operatorname{tr}: R^{1} \pi_{*} \mathscr{K}_{2} \rightarrow \mathcal{O}^{*}$ transforms $\mathrm{IC}_{2}(E)$ into $\mathcal{O}^{*}$-torsor $\mathrm{IC}_{2}$. We would like to have a similar construction for $\operatorname{det} R \pi_{*} E$. Certainly, the corresponding extension of $\mathrm{GL}_{n}$ is functorial for étale maps only, so one should also vary $X$ and add to the gauge group $\mathrm{GL}_{n}$ the "diffeomorphisms," as is indicated by the Lie algebra analogue. For example, one may consider the category $\mathrm{G}$ whose objects are pairs $(U, t)$, where $U$ is a smooth curve and $t \in \mathcal{O}(U)$ is a coordinate function (i.e. $d t$ is invertible); a morphism $\varphi:(U, t) \rightarrow\left(U^{\prime}, t^{\prime}\right)$ is a pair $\left(\varphi_{U}, \varphi_{g}\right)$, where $\varphi_{U}: U \rightarrow U^{\prime}$ is an étale map and $\varphi_{g} \in \mathrm{GL}_{n}(\mathcal{O}(U))$; we have $\varphi^{\prime} \cdot \varphi=\left(\varphi_{U^{\prime}}^{\prime} \cdot \varphi_{U^{\prime}}\left(\varphi_{g}^{\prime} \cdot \varphi_{U}\right) \cdot \varphi_{g}\right)$. We would like to have the extension $\mathrm{G}$ of this category by $\mathscr{K}_{2}$ [or rather by $\mathscr{K}_{2} / K_{2}$ (constants)], so as its Lie algebra would coincide with the Virasoro algebra. If one has a curve $X$ with a vector bundle $E$, then we may take an atlas $U_{i}, \cup U_{i}=X$ with coordinates $t_{i} \in \mathcal{O}\left(U_{i}\right)$ and trivialisations of $\left.E\right|_{U_{i}}$. These data determine the morphisms $\varphi_{i j}:\left(U_{i j}, t_{i}\right)$ $\rightarrow\left(U_{i j}, t_{j}\right)$ in $\mathrm{G}$ such that $\varphi_{i j} \varphi_{j k}=\varphi_{i k}$ for any $i, j, k$. The liftings $\hat{\varphi}_{i j}$ of $\varphi_{i j}$ to $\hat{\mathrm{G}}$ with the 
same cocycle property form a $H^{1}\left(X, \mathscr{K}_{2} / K_{2}(\mathbb{C})\right.$ )-torsor, which should be canonically isomorphic to det $R \pi_{*} E$ (or rather to its 12 -th power); the tangent map to this isomorphism should be 2.3. The RR-isomorphism should come just from the isomorphism between $\hat{\mathrm{G}}$ and the sum of $\hat{\mathrm{GL}}_{n}$ above with the analogous extension of $\mathcal{O}^{*} \times \mathcal{O}^{*}$ that corresponds to $\mathrm{c}_{1} \cdot \mathrm{c}_{1}$ in the right-hand the side of $\mathrm{RR}$. Unfortunately, at a moment we can only perform this on the Lie algebra level, see Appendix.

\subsection{Higher-Dimensional Version}

Now we will sketch very briefly the higher-dimensional version of 2.3 ; unfortunately, no canonical complexes are known here, so one should swamp in the derived category. For brevity, we assume that $E=\mathcal{O}_{X}$.

So let $\pi: X \rightarrow S$ be a smooth proper map of relative dimension $n$; denote $\omega=\Omega_{X / S}^{n}$, and let $\Delta: X \rightarrow X \times{ }_{S} X$ be the diagonal map. Then, following Grothendieck and Sato, we may identify $\mathscr{D}_{X / S}$ (= the differential operators along the fibers of $\pi)$ with $H_{\Delta}^{n}\left(X \times X, p_{2}^{*} \omega\right)$.

In particular, we have the canonical morphism $\mathscr{A}_{X / S} \rightarrow p_{2}^{*} \omega[n]$, hence the triangle $\mathscr{B} \rightarrow \mathscr{A}_{X / S} \rightarrow p_{2}^{*} \omega[n]$. Let $\mathscr{C}$ be the one of the composition $\mathscr{B} \rightarrow \mathscr{A}_{X / S} \rightarrow \mathscr{A}_{X, \pi}$, so that we have the triangle $p_{2}^{*} \omega[n] \rightarrow \mathscr{C} \rightarrow \mathscr{T}_{\pi}$. Then one has the canonical isomorphism $R(\pi \times \pi)_{*} \mathscr{C} \cong \mathscr{A}_{R \pi_{*} \mathcal{O}_{X}}$ [where $\mathscr{A}_{R \pi_{*} \mathcal{O}_{X}}$ is an appropriately defined 2-step filtered object, the extension of $\mathscr{T}_{S}$ by $\left.R \operatorname{End}\left(R \pi_{*} \mathcal{O}_{X}\right)\right]$. The role of $\mathscr{P}$ is now played by the cone of $\mathscr{D}_{X / S} \rightarrow p_{2}^{*} \omega[n]$, completed at $\Delta$ : so one has the exact triangle $p_{2}^{*} \omega_{\hat{\Delta}}[n-1] \rightarrow \mathscr{P}_{\rightarrow} \rightarrow \mathscr{D}_{X / S}$. The appropriate subquotient of $\mathscr{P}$ is an analogue of Virasoro algebra $\mathscr{H}_{X / S}^{n}[n-1] \rightarrow \mathscr{\mathscr { D }}_{X / S} \rightarrow \mathscr{D}_{X / S}$, and one has a canonical isomorphism $R^{0} \pi_{*} \operatorname{Cone}\left(\hat{\mathscr{A}}_{X / S} \rightarrow \mathscr{A}_{X, \pi}\right) \cong \mathscr{A}_{\operatorname{det} R \pi_{*} \boldsymbol{O}_{X}}$.

For the higher-dimensional version of Tate's construction see [2].

\section{Differential Equations on Mumford Forms}

\subsection{Mumford Forms}

Note that $\mathscr{T}_{\pi}$ acts on $\Omega_{X / S}^{1 \otimes j}=\omega^{j}$ via Lie derivative: we have a Lie algebra homomorphism $\operatorname{Lie}_{j}: \mathscr{T}_{\pi} \rightarrow \mathscr{A}_{\omega^{j}, \pi}, \varepsilon \circ \operatorname{Lie}_{j}=\mathrm{id}_{\mathscr{T}_{\pi}}$. Define $\mathscr{A}_{j} C^{\text {tr }} \mathscr{A}_{\omega^{j}}$ to be the $\pi$-subalgebra that coincides with $\operatorname{Lie}_{j}\left(\mathscr{T}_{\pi}\right)$ in degree 0 . Hence $\mathscr{A}_{j}$ is an $\Omega$-extension of $\mathscr{T}_{\pi}$ and 2.3 identifies $\pi\left(\mathscr{A}_{j}\right)$ with $\mathscr{A}_{\lambda}$, where $\lambda_{j}:=\lambda_{\omega^{j}}$. Here is an explicit description of $\mathscr{A}_{j}$.

A local coordinate $t$ (along the fibres) determines the isomorphism $s_{j t}: \mathcal{O}_{X} \oplus \mathcal{O}_{X} \stackrel{\sim}{\rightarrow} \mathscr{A}_{j}^{-1}$,

$$
\begin{aligned}
s_{j t}(\chi, v) & =(\chi, v)_{j t} \\
& =\left(\frac{\chi\left(t_{1}\right)}{\left(t_{2}-t_{1}\right)^{2}}+\frac{j \chi^{\prime}\left(t_{1}\right)}{t_{2}-t_{1}}+(j / 2-1 / 6) \chi^{\prime \prime}\left(t_{1}\right)+v\left(t_{1}\right)\right) d t_{1}^{j} d t_{2}^{1-j} .
\end{aligned}
$$

One has the formulas for bracket [here $\tau \in \mathscr{T}_{\pi}=\mathscr{A}_{j}^{0}, \tau=\tau_{1}(t, s) \partial_{t}+\tau_{2}(s) \partial_{s}$, $\left.\tau^{\prime}=\partial_{t}\left(\tau_{1}\right), c_{j}=6 j^{2}-6 j+1\right]$ :

$$
\begin{gathered}
{\left[\tau,(\chi, v)_{j t}\right]=\left(\left[\tau, \chi \partial_{t}\right], \tau(v)+v \tau^{\prime}-1 / 6 c_{j} \chi^{\prime} \tau^{\prime \prime}\right)_{j t},} \\
{\left[\left(\chi_{1}, v_{1}\right)_{j t},\left(\chi_{2}, v_{2}\right)_{j t}\right]=-1 / 6 c_{j}\left(\chi_{1}^{\prime} \chi_{2}^{\prime}+\chi_{1} v_{2}+\chi_{2} v_{1}\right) .}
\end{gathered}
$$


Change of coordinates: let $y=y(t)$ be another coordinate. Then

$$
(\chi, v)_{j y}=\left(\chi / y^{\prime}, v y^{\prime}+\frac{c_{j}}{12} \frac{y^{\prime \prime}}{y^{\prime}}\left(\chi \frac{y^{\prime \prime}}{y^{\prime 2}}-2 \chi^{\prime}\right)\right)_{j t} .
$$

These formulas determine $\mathscr{A}_{j}$ completely, and imply

3.1.1. Lemma-Construction. We have a canonical isomorphism of $\pi$-algebras ${ }^{\#} \mu_{j}: \mathscr{A}_{j} \stackrel{\sim}{\rightarrow} c_{j} \mathscr{A}_{i}$ such that ${ }^{\#} \mu_{j}(\chi, v)_{j t}=(\chi, v)_{1 t}^{\left(c_{j}\right)}:=$ the image of $(\chi, v)_{1 t}$ under the map $\mathscr{A}_{1}^{-1} \rightarrow\left(c_{j} \mathscr{A}_{1}\right)^{-1}$.

Hence we get a canonical isomorphism $\mathscr{A}_{\lambda_{j}} \cong c_{j} \mathscr{A}_{\lambda_{1}}$, or, equivalently, the integrable connection $\nabla_{j}$ on $\lambda_{j} \otimes \lambda_{1}^{-c_{j}}$.

3.1.2. $\nabla_{j}$ corresponds to Mumford's trivialisation of this bundle: it has a non-zero horizontal section $\mu_{j}$ which is just Mumford's discriminant form [17].

To see this (the following arguments are parallel to those from [17]), note that we may assume, since our connection is natural, that $X$ is a universal family of genus $g$ curves, so $S=\mathscr{M}_{g}=$ moduli space of genus $g$ curves. For $g \geqq 3 \mathscr{M}_{g}$ admits a compactification $\overline{\mathscr{M}}_{g}$ (the closure of $\mathscr{M}_{g}$ in Satake compactified moduli of abelian varieties) such that codim $\overline{\mathscr{M}}_{g} \backslash \mathscr{M}_{g} \geqq 2$, and $H_{1}\left(\mathscr{M}_{g}, \mathbb{Z}\right)=0$. Hence, by Deligne's theorem, any integrable connection on a bundle on $\mathscr{M}_{g}$ has regular singularities at infinity, and so is determined by a global monodromy. Since $H_{1}\left(\mathscr{M}_{g}, \mathbb{Z}\right)=0$, any rank 1 vector bundle with integrable connection is trivial (and any of its global section is horizontal). This proves our assertion for $g \geqq 3$. If $g=1,2$ any curve is hyperelliptic, and we will calculate $\mu_{j}$ in the next subsection.

3.1.3. Remark. The transposition of coordinates defines a canonical isomorphism $\mathscr{A}_{j} \cong \mathscr{A}_{1-j}\left[\right.$ it maps $(\chi, v)_{j t}$ into $\left.(\chi, v)_{1-j, t}\right]$ the corresponding isomorphism $\mathscr{A}_{\lambda_{j}} \cong \mathscr{A}_{\lambda_{1-},}$ comes from Serre's duality isomorphism $\lambda_{j} \cong \lambda_{1-j}$.

Lemma 3.1.1 is a particular case of general Riemann-Roch isomorphism for Virasoro algebras, see Appendix, A 3.3.

3.1.4. Let $\tau$ be a vector field on $S$. To calculate $\nabla_{j}(\tau)$ one may proceed, according to 2.3.3, as follows. We will work locally on $S$. Choose a section $q=q(s)$ of $\pi$ ( $=S$-point of $X$ ); let $U$ be a neighbourhood of $q$ with a coordinate function $t$ [along the fibers; $t(q)=0]$; put $\dot{X}:=X \backslash q(S), \dot{U}:=U \backslash q(S)$. Fix some liftings $\tilde{\tau}_{X} \in \mathscr{T}_{\pi}(\dot{X})$, $\tilde{\tau}_{U} \in \mathscr{T}_{\pi}(U)$ of $\tau$ to $\dot{X}, U$ respectively: $d \pi(\tilde{\tau})=\tau$; put $\tau^{\vee}:=\tilde{\tau}_{X}-\tilde{\tau}_{U} \in \mathscr{T}_{X / S}(\dot{U})$. For $j \geqq 1$ choose a section $r_{j} \in \omega^{j} \bowtie \omega^{1-j}(2 \Delta)\left(X \times{ }_{S} \dot{U}\right)$ such that $\delta\left(r_{j}\right)=\operatorname{Lie}_{j}\left(\tau^{\vee}\right) \in \mathscr{A}_{\omega^{j} / S}(\dot{U})$ $\subset \mathscr{A}_{\omega^{3}, \pi}(\dot{U})$. Then $\left(\tilde{\tau}_{X}, r_{j}\right)$ is a cocycle from $(2.3 .3 .3)$, which acts on the vector bundle $\pi_{*}\left(\omega^{j}\right)$ by formula $(2.3 .3 .4)$ :

$$
\tau_{j}\left(\varphi_{(j)}\right):=\left(\tilde{\tau}_{X}, r_{j}\right)\left(\varphi_{(j)}\right)=\operatorname{Lie}_{j}\left(\tilde{\tau}_{X}\right)\left(\varphi_{(j)}\right)+\operatorname{Res}_{t_{2}=0} r_{j}\left(t, t_{2}\right) \varphi_{(j)}\left(t_{2}\right),
$$

where $\varphi_{(j)} \in \pi_{*}\left(\omega^{j}\right)$ and the residue is taken over the second coordinate. The trace action of $\tau_{j}$ on $\operatorname{det} \pi_{*}\left(\omega^{j}\right)=\lambda_{j}$ is given by the formula

$$
\begin{aligned}
& \left(\operatorname{tr} \tau_{j}\right)\left(\operatorname{det} \varphi_{(j)}^{i}\right) \\
& =\tau_{j}\left(\varphi_{(j)}^{1}\right) \wedge \varphi_{(j)}^{2} \wedge \ldots+\varphi_{(j)}^{1} \wedge \tau_{j}\left(\varphi_{(j)}^{2}\right) \wedge \ldots+\ldots=\alpha_{j} \operatorname{det} \varphi_{(j)}^{i},
\end{aligned}
$$


$\left\{\varphi_{(j)}^{i}\right\}$ is a basis of $\pi_{*}\left(\omega^{j}\right)$. Thus $\operatorname{tr} \tau_{j}$ corresponds by 2.3.3.5 to the cocycle $\left(\tilde{\tau}_{X}, \beta\left(r_{j}\right)\right)$ of $\mathscr{A}_{j}$. Consider the expansion of $r_{j}$ in a neighbourhood of $\Delta$ :

$$
r_{j}\left(t_{1}, t_{2}\right)=\left(\frac{\tau^{\vee}\left(t_{1}\right)}{\left(t_{2}-t_{1}\right)^{2}}+\frac{j \tau^{\vee}\left(t_{1}\right)^{\prime}}{t_{2}-t_{1}}+l_{j}\left(t_{1}\right)\right) d t_{1}^{j} d t_{2}^{1-j}+o\left(t_{2}-t_{1}\right) .
$$

Then for the generator $\varphi=\operatorname{det} \varphi_{(j)}^{i} /\left(\operatorname{det} \varphi_{(1)}^{i}\right)^{c_{j}}$ of $\lambda_{j} / \lambda_{1}^{c_{j}}$, one has

$$
\nabla_{j}(\tau)(\varphi)=\left[\alpha_{j}-\operatorname{Res}_{t=0} l_{j}(t) d t-c_{j}\left(\alpha_{1}-\operatorname{Res}_{t=0} l_{1}(t) d t\right)\right] \varphi .
$$

\subsection{Hyperelliptic Case}

Let $X_{a}$ be a hyperelliptic curve

$$
y^{2}=P_{a}(x)=\prod_{i=1}^{2 g+2}\left(x-a_{i}\right), a=\left(a_{i}\right) \in \mathbb{C}^{2 g+2}, a_{i} \neq a_{j}
$$

for $i \neq j ; \sigma: X_{a} \rightarrow X_{a}$ the involution $\sigma(y, x)=(-y, x)$. So $X_{a}$ is a covering of $\mathbb{P}^{1}$ ramified at $\left\{a_{i}\right\}$ and $X$ is a universal family of hyperelliptic curves parametrized by $S=\mathbb{C}^{2 g+2} \backslash\{$ diagonals $\}$. Put $\varphi=\frac{d x}{y} \in \omega(X), \operatorname{div} \varphi=(g-1) \operatorname{div}_{\infty}(x)$. We have the following explicit base $B_{j}$ in $\omega^{j}(X)$ :

$$
\begin{gathered}
B_{1}=\left\{\varphi, x \varphi, \ldots, x^{g-1} \varphi\right\}, \\
B_{j}=\left\{\varphi^{j}, x \varphi^{j}, \ldots, x^{j(g-1)} \varphi^{j} ; y \varphi^{j}, y x \varphi^{j}, \ldots, y x^{(j-1)(g-1)-2} \varphi^{j}\right\} \text { for } j>1 .
\end{gathered}
$$

3.2.1. Lemma. $\mu_{j}=$ const $\cdot \prod_{\substack{(i, k) \\ i \neq k}}\left(a_{i}-a_{k}\right)^{\frac{j(j-1)}{2}} \operatorname{det} B_{j} /\left(\operatorname{det} B_{1}\right)^{c_{j}}$.

Proof. We will follow the lines of 3.1.4. We have to show that the $\mu_{j}$ so defined satisfies $\nabla_{j}\left(\partial_{a_{\imath}}\right)\left(\mu_{j}\right)=0$ (for any $i$ ).

Put $\tau=\partial_{a_{i}}, q=\left(a_{i}, 0\right) \in X$, so $y$ is a coordinate at $q, \tilde{\tau}_{X} \in \mathscr{T}_{\pi}(\dot{X})$ is the lifting of $\tau$ such that $\tilde{\tau}_{\dot{X}}(x)=0: \tilde{\tau}_{X}=-\frac{1}{2} \frac{y \partial y}{x-a_{i}}, \tau^{v}:=-\frac{P^{\prime}\left(a_{i}\right)}{2 y} \partial_{y}=-\frac{P^{\prime}\left(a_{i}\right)}{P^{\prime}(x)} \partial_{x}$ (here $P^{\prime}\left(a_{i}\right)$ $\left.=\prod_{k \neq i}\left(a_{i}-a_{k}\right)\right), \tilde{\tau}_{U}=\tilde{\tau}_{X}-\tau^{v}=\left(\prod_{k \neq i}\left(x-a_{k}\right)+\prod_{k \neq i}\left(a_{i}-a_{k}\right)\right) \frac{d y}{2 y}$.

Formula for $r_{j}$ :

$$
\begin{aligned}
& r_{j}\left(\left(x_{1}, y_{1}\right),\left(x_{2}, y_{2}\right)\right)=\frac{1}{2}\left(-\frac{\left(y_{1}+y_{2}\right) P^{\prime}\left(a_{i}\right)}{2 P^{\prime}\left(x_{2}\right)\left(x_{2}-x_{1}\right)^{2}}+\frac{P^{\prime}\left(a_{i}\right)}{4 y_{2}\left(x_{2}-x_{1}\right)}\right. \\
& \left.+\frac{j-1}{4} \frac{P^{\prime}\left(a_{i}\right)\left(y_{1}+y_{2}\right)\left(2 P^{\prime \prime}\left(x_{2}\right) P\left(x_{2}\right)+P^{\prime}\left(x_{2}\right)^{2}\right)}{P\left(x_{2}\right) P^{\prime}\left(x_{2}\right)^{2}\left(x_{2}-x_{1}\right)}\right)\left(\frac{d x_{1}}{y_{1}}\right)^{j}\left(\frac{d x_{2}}{y_{2}}\right)^{1-j} .
\end{aligned}
$$

This formula may be found as follows. Let $\Delta^{\prime}:=(1, \sigma)(\Delta)$. The function $y_{1}+y_{2}$ equals $2 y$ on $\Delta$ and vanishes on $\Delta^{\prime}$. Hence the first summand of the formula has pole of order 2 at $\Delta$ with the right higher term, and the pole of order 1 at $\Delta^{\prime}$. The second summand kills the pole at $\Delta^{\prime}$. The third summand is regular at $\Delta^{\prime}$ and corrects the first order pole component at $\Delta$ to have $\delta\left(r_{j}\right)=\tau^{v}$. Finally, it remains to note that $r_{j}$ is regular at $x_{1}=\infty$. 
Now we may use the formula

$$
\begin{aligned}
y_{1} / y_{2}=1 & +\frac{1}{2} \frac{P^{\prime}\left(x_{2}\right)}{P\left(x_{2}\right)}\left(x_{1}-x_{2}\right) \\
& +\left(\frac{1}{4} \frac{P^{\prime \prime}\left(x_{2}\right)}{P\left(x_{2}\right)}-\frac{1}{8} \frac{P^{\prime}\left(x_{2}\right)^{2}}{P\left(x_{2}\right)^{2}}\right)\left(x_{1}-x_{2}\right)^{2}+o\left(x_{1}-x_{2}\right)^{2}
\end{aligned}
$$

to calculate the expansion of $r_{j}$ at $\Delta$ :

$$
\begin{aligned}
r_{j}= & \frac{1}{2}\left(\frac{-P^{\prime}\left(a_{i}\right)}{P^{\prime}\left(x_{2}\right)} \frac{1}{\left(x_{2}-x_{1}\right)^{2}}+(j-1) \frac{P^{\prime}\left(a_{i}\right) P^{\prime \prime}\left(x_{2}\right)}{P^{\prime}\left(x_{2}\right)^{2}} \frac{1}{x_{1}-x_{2}}\right. \\
& +\left(\frac{j^{2}}{8}+\frac{j}{8}+\frac{3}{16}\right) \frac{P^{\prime}\left(a_{i}\right) P^{\prime}\left(x_{2}\right)}{P\left(x_{2}\right)^{2}}+\left(\frac{j^{2}}{2}-\frac{j}{2}+\frac{1}{8}\right) \\
& \left.\times \frac{P^{\prime}\left(a_{i}\right) P^{\prime \prime}\left(x_{2}\right)}{P\left(x_{2}\right) P^{\prime}\left(x_{2}\right)}+o\left(x_{1}-x_{2}\right)\right) d x_{1}^{j} d x_{2}^{1-j}
\end{aligned}
$$

Hence

$$
\begin{aligned}
\beta\left(r_{j}\right)= & \frac{1}{2}\left(\frac{-P^{\prime}\left(a_{i}\right)}{P^{\prime}\left(x_{1}\right)} \frac{1}{\left(x_{2}-x_{1}\right)^{2}}+\frac{j P^{\prime}\left(a_{i}\right) P^{\prime \prime}\left(x_{1}\right)}{P^{\prime}\left(x_{1}\right)^{2}\left(x_{2}-x_{1}\right)}\right) d x_{1}^{j} d x_{2}^{1-j} \\
& +\frac{1}{4}(2 j-1)^{2} \sum_{k \neq i} \frac{1}{a_{i}-a_{k}} \frac{d y}{y}+\text { exact form },
\end{aligned}
$$

since

$$
\operatorname{Res} \frac{P^{\prime}}{P^{2}} d x=0, \operatorname{Res} \frac{P^{\prime \prime}}{P P^{\prime}} d x=\frac{4}{P^{\prime}\left(a_{i}\right)} \sum_{k \neq i} \frac{1}{a_{i}-a_{k}} .
$$

So

$$
l_{j} d y=\frac{1}{4}(2 j-1)^{2} \sum \frac{d y}{a_{i}-a_{k}}, \quad \operatorname{Res}_{y=0} l_{j} d y=\frac{1}{4}(2 j-1)^{2} \sum \frac{1}{a_{i}-a_{k}}
$$

(see 3.1.4.1).

Action of $\tau_{j}$ on $\pi_{*} \omega^{j}:$ we have $\tilde{\tau}_{X}(y)=-\frac{1}{2} \frac{y}{x-a_{i}}, \tilde{\tau}_{\dot{X}}(x)=0$;

$$
\begin{gathered}
\operatorname{Lie}_{j}\left(\tilde{\tau}_{X}\right)\left(\alpha(x) \varphi^{j}\right)=\frac{j \alpha(x) \varphi^{j}}{2\left(x-a_{i}\right)}, \\
\operatorname{Lie}_{j}\left(\tilde{\tau}_{X}\right)\left(y \beta(x) \varphi^{j}\right)=\frac{(y-1) y \beta(x) \varphi^{j}}{2\left(x-a_{i}\right)}, \\
\operatorname{Res}_{y_{2}=0}\left(r_{j}\left(\left(x_{1}, y_{1}\right),\left(x_{2}, y_{2}\right)\right) \alpha\left(y_{2}\right) \varphi^{j}\left(x_{2}, y_{2}\right)\right)=\frac{j}{2} \alpha\left(a_{i}\right) \frac{1}{a_{i}-x} \varphi^{j}, \\
\operatorname{Res}_{y_{2}=0}\left(r_{j}\left(\left(x_{1}, y_{1}\right),\left(x_{2}, y_{2}\right)\right) y_{2} \beta\left(x_{2}\right) \varphi^{j}\left(x_{2}, y_{2}\right)=\frac{j-1}{2} \beta\left(a_{i}\right) \frac{y}{a_{i}-x} \varphi^{j} .\right.
\end{gathered}
$$


These formulas imply that the trace of the matrix that gives the action of $\tau_{j}$ in basis $B_{j}$ is 0 . Hence $\operatorname{tr} \tau_{j}\left(\operatorname{det} B_{j}\right)=0$. This, together with the above formula for $\operatorname{Res} l_{j}$ gives the differential equation for the Mumford form

$$
\mu_{j}=\mu_{j}(a) \operatorname{det} B_{j} / \operatorname{det} B_{1}^{c_{j}}:\left(\partial_{a_{t}}-\frac{j(j-1)}{2} \sum \frac{1}{a_{i}-a_{k}}\right) \mu_{j}(a)=0,
$$

which implies the lemma.

Compare with field-theoretic computations in [23]; for the generalisation to $Z / n$-coverings of $\mathbb{P}^{1}$ see $[8,13]$.

\subsection{Problem}

It would be very interesting to calculate the expansion of $\mu_{j}$ near the boundary of moduli space, e.g. at points that correspond to a completely degenerate stable curve: these are in $1-1$ correspondence with connected graphs of $2 g-2$ vertices and $3 g-3$ edges, with exactly 3 edges at any vertex; here $g=$ genus. Case

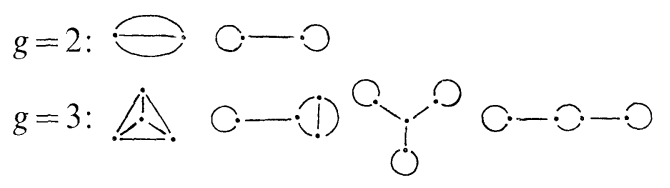

and to calculate $\mu_{j}$ in terms of Schottki uniformisation (one should get the formulas similar to [1]).

\subsection{Remark on Cancellation of Anomalies in Higher Dimensions}

In situation 2.8 denote by $f_{j}\left(\mathrm{c}_{1}, \ldots, \mathrm{c}_{n}\right)$ the term of power $n+1$ of the series $e^{-j \mathrm{c}} \mathrm{Td}\left(\mathrm{c}_{1}, \ldots, \mathrm{c}_{n}\right)$, where we put deg $\mathrm{c}_{i}=i, \mathrm{Td}$ is the Todd genus. Examples: $f_{j}^{(1)}\left(\mathrm{c}_{1}\right)$ $=\left(6 j^{2}-6 j+1\right) c_{1}^{2} ; f_{j}^{(2)}\left(c_{1}, c_{2}\right)=-j / 12 \cdot c_{1}\left(c_{2}+(j-1)(2 j-1) c_{1}^{2}\right)$.

Suppose that integers $a_{1}, \ldots, a_{N}, j_{1}, \ldots, j_{N}$ are such that $\sum_{i=1}^{N} a_{i} f_{j_{l}}^{(n)}=0$. Then by Grothendieck-Riemann-Roch formula we have $c_{1}\left(\otimes \lambda_{j_{i}}^{a_{2}}\right)=0$, where $\lambda_{j}:=\operatorname{det} R \pi_{*} \omega^{\otimes j}$. It would be interesting to prove a generalisation of (3.1.1) which should imply the existence of canonical connections on sheaves $\otimes \lambda_{j_{l}}^{a_{2}}$; maybe these sheaves are trivial?

\section{Virasoro Action on Moduli Spaces}

\subsection{Enhanced Moduli Spaces}

Let $m, n \geqq 1, g \geqq 0, d$ be some integers. Denote by $\widehat{\mathscr{M}}=\hat{\mathscr{M}}_{g, m}$ the moduli space of the data $\left(X ; x_{1}, \ldots, x_{m} ; t_{1}, \ldots, t_{m}\right)$, where $X$ is a genus $g$ curve, $x_{1}, \ldots, x_{m} \in X$ are different points, and $t_{i}$ are formal parameters at $x_{i}$. Similarly, let $\hat{\mathscr{M}}^{\prime}=\hat{\mathscr{M}}_{g, m}^{n . d}$ be the moduli space of the data $\left(X, E ; x_{1}, \ldots, x_{m} ; t_{1}, \ldots, t_{m} ; I_{1}, \ldots, I_{m}\right)$, where $X, x_{i}, t_{i}$ are as above, $E$ is a rank $n$ vector bundle on $X$ of degree $d$, and $I_{\imath}: \hat{\mathcal{O}}_{x_{\imath}}^{n} \stackrel{\sim}{\rightarrow} \hat{E}_{x_{\imath}}$ are formal 
trivialisations of $E$ at $x_{i}$. Both $\mathscr{M}$ and $\mathscr{M}^{\prime}$ are projective limits of smooth varieties $\mathscr{M}^{(k)}, \mathscr{M}^{\prime(k)}=$ moduli of the same sort of data with $t_{i}, I_{i}$ are jets of trivialisations of certain finite order $k$, with respect to obvious smooth projections; so $\mathscr{M}, \mathscr{M}^{\prime}$ are schemes (of infinite type). Let $\pi: X \rightarrow \mathscr{M}, \pi^{\prime}: X^{\prime} \rightarrow \mathscr{M}^{\prime}$ be the universal curves, $E$ be the universal bundle on $X^{\prime}$, etc. Put $\mathscr{L}:=\mathbb{C}((t)) \partial_{t}, \mathscr{L}^{\prime}:=\mathscr{A}_{\text {On }}(\operatorname{Spec} \mathbb{C}((t)))$ $=\mathbb{C}((t))\left(\partial_{t}+\mathrm{Mat}_{n}\right)$ : these are our usual Lie algebras on the punctured coordinate disc. Consider the maps

$$
\varphi=\left(\varphi_{i}\right): \mathscr{T}_{\pi}(\dot{X}) \rightarrow \mathscr{L}^{m} \hat{\otimes} \mathcal{O}_{\hat{M}}, \quad \varphi^{\prime}: \mathscr{A}_{E, \pi}\left(X^{\prime}\right) \rightarrow \mathscr{L}^{\prime m} \hat{\otimes} \mathcal{O}_{\hat{M}^{\prime}}
$$

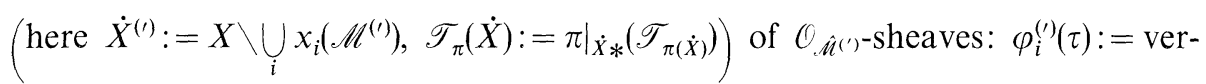
tical component of $\tau$ with respect to $t_{i}$ or $t_{i}, I_{i}$ [if $\tau=\tau^{h}(s) \partial_{s}+\tau^{v}\left(t_{i}, s\right)$ near $x_{i}$, then $\left.\varphi_{i}(\tau)=\tau^{v}\left(t_{i}, s\right)=\tau\left(t_{i}\right) \partial_{t}\right]$.

4.1.1. Lemma. $\varphi, \varphi^{\prime}$ are isomorphisms.

Proof. The exact sequences $\quad 0 \rightarrow \mathscr{T}_{\mid \hat{M}}(\dot{X}) \rightarrow \mathscr{T}_{\pi}(\dot{X}) \rightarrow \mathscr{T}_{\hat{M}} \rightarrow 0, \quad 0 \rightarrow \mathscr{A}_{E / \hat{M}^{\prime}}\left(\dot{X}^{\prime}\right)$ $\rightarrow \mathscr{A}_{E, \pi}\left(\dot{X}^{\prime}\right) \rightarrow \mathscr{T}_{\hat{M}^{\prime}} \rightarrow 0$ show that it suffices to prove that $\varphi, \varphi^{\prime}$ induce isomorphisms

$$
\mathscr{T}_{\hat{\mathscr{M}}} \stackrel{\sim}{\rightarrow} \mathscr{L}^{m} \hat{\otimes} \mathcal{O}_{\hat{M}} / \varphi\left(\mathscr{T}_{\mid \mathcal{M}}(\dot{X})\right), \mathscr{T}_{\hat{M}^{\prime}} \tilde{\rightarrow}^{\prime m} \hat{\otimes} \hat{\mathcal{O}}_{\mathscr{M}} / \varphi^{\prime}\left(\mathscr{A}_{E / \hat{M}^{\prime}}\left(\dot{X}^{\prime}\right)\right) .
$$

But these are just Kodaira-Spencer maps for our moduli problem, hence the result.

So $\varphi^{-1}, \varphi^{\prime-1}$ define the embeddings $\mathscr{L}^{m} \hookrightarrow \mathscr{T}_{\pi}(\dot{X}), \mathscr{L}^{\prime m} \hookrightarrow \mathscr{A}_{E, \pi}\left(\dot{X}^{\prime}\right)$. They commute with brackets (as follows from the definition), therefore we have a $\mathscr{L}^{m}$-action on $X$ and $\mathscr{L}^{\prime m}$-action on $X^{\prime}$ compatible with projections $\pi$, $\pi^{\prime}$ hence the corresponding actions on $\hat{\mathscr{M}}, \hat{\mathscr{M}}^{\prime}$. Clearly, the obvious projections $\dot{X}^{\prime} \rightarrow X, \hat{\mathscr{M}}^{\prime} \rightarrow \hat{\mathscr{M}}^{\prime}$ are equivariant with respect to $\varepsilon: \mathscr{L}^{\prime} \rightarrow \mathscr{L}$. Lemma also implies that these actions are infinitesimally transitive, i.e. the elements of the Lie algebra generate the tangent plane at any point. More precisely, for any point $m^{(\prime)} \in \hat{\mathscr{M}}^{(\prime)}$ we have canonical short exact sequences

$$
0 \rightarrow \mathscr{T}\left(\dot{X}_{m}\right) \rightarrow \mathscr{L}^{m} \rightarrow \mathscr{T}_{\hat{\mathcal{M}}, m} \rightarrow 0, \quad 0 \rightarrow \mathscr{A}_{E m^{\prime}}\left(\dot{X}_{m^{\prime}}^{\prime}\right) \rightarrow \mathscr{L}^{\prime m} \rightarrow \mathscr{T}_{\hat{M}^{\prime}, m^{\prime}} \rightarrow 0
$$

the left arrows are the Laurent series expansions at $x_{i}$ with respect to $t_{i}\left(I_{i}\right)$, the right ones correspond to action (= Kodaira-Spencer maps). Note that the action of the positive part $\mathscr{L}^{+m}:=\left(t \mathbb{C}[[t]] \partial_{t}\right)^{m} \subset \mathscr{L}^{m}$ just changes $t_{i}$, the action of $\partial_{t}^{(i)}$ moves $x_{i}$ (this part of the construction links with the "formal geometry" of Gelfand,...), and it is the "polar" part that changes the complex structure of the curve.

Consider the determinant bundles $\lambda:=\lambda_{O_{x}}, \lambda^{\prime}:=\lambda_{E}$ on $\hat{\mathscr{M}}, \hat{\mathscr{M}}^{\prime}$ respectively. Let $\hat{\mathscr{L}}, \hat{\mathscr{L}}^{\prime}$ be the "coordinate" Virasoro algebras, the central extensions of $\mathscr{L}, \mathscr{L}^{\prime}$ by $\mathbb{C}$, and $\hat{\mathscr{L}}^{m}, \hat{\mathscr{L}}^{\prime m}$ be the sums of $m$ copies of these extensions (= extensions of $\mathscr{L}^{()^{m}}$ by $\mathbb{C})$. Now 2.3 implies that the $\mathscr{L}^{(\prime) m}$ action on $\hat{\mathscr{M}}^{(\prime)}$ lifts canonically to the Virasoro algebras action on $\lambda, \lambda^{\prime}$ with central charge 1 . To see this one should calculate $\pi\left({ }^{\mathrm{tr}} \mathscr{A}_{\mathscr{C}_{X}}\right), \pi\left({ }^{\mathrm{tr}} \mathscr{A}_{E}\right)$ by means of adéle, or formal Čech, cochains of the covering $\left\{U, \dot{X}^{(\prime)}\right\}$ of $X^{(\prime)}$, where $U:=\bigcup_{i} U_{i}$ is the union of formal neighbourhoods of $x_{i}$. The cochains (in $\lambda^{\prime}$ case) are the triples

$$
\left\{\tau_{X}, \tau_{U} ; r_{U}\right\}, \tau_{X} \in \mathscr{A}_{E, \pi}(\dot{X}), \tau_{U} \in \mathscr{A}_{E, \pi}(U)=\prod_{i} \mathscr{A}_{E, \pi}\left(U_{i}\right)
$$


$r_{U} \in^{\operatorname{tr}} \mathscr{A}_{E}^{-1}(\dot{U})=\hat{\mathscr{L}}^{\prime m} \hat{\otimes} \mathcal{O}_{\hat{M}^{\prime}}$ such that $\tau_{X}-\tau_{U}=\delta\left(r_{U}\right)$; the bracket is given by the formula $\left[\left(\tau_{X}^{1}, \tau_{U}^{1} ; r_{U}^{1}\right),\left(\tau_{X}^{2}, \tau_{U}^{2} ; r_{U}^{2}\right)\right]=\left(\left[\tau_{X}^{1}, \tau_{\dot{X}}^{2}\right],\left[\tau_{U}^{1}, \tau_{U}^{2}\right] ;\left[r_{U}^{1}, \tau_{U}^{2}\right]+\left[\tau_{\dot{X}}^{1}, r_{\dot{U}}^{2}\right]\right)$. It is easy to see that the formula $r \mapsto\left(\varphi^{\prime-1}(r),-\delta(r)+\varphi^{\prime-1}(r) ; r\right)$ defines the Lie algebra map $\hat{\mathscr{L}}^{\prime m} \rightarrow \pi\left({ }^{\text {tr }} \mathscr{A}_{E}\right)$ which is, according to 2.3 , the desired action of $\hat{\mathscr{L}}^{\prime m}$ on $\lambda^{\prime}$; the $\hat{\mathscr{L}}^{m}$ action on comes similarly.

We may rephrase 3.1.1 as follows: $\hat{\mathscr{L}}^{m}$ also acts canonically on $\lambda_{j}=\operatorname{det} R \pi_{*} \omega^{j}$ with central charge $c_{j}$, and Mumford form $\mu_{j}$ is just the $\hat{\mathscr{L}}^{m}$-invariant section of $\lambda_{j} / \lambda_{1}^{\otimes c_{j}}$.

\subsection{Generalities on $((\mathfrak{5}, K)$-Schemes}

We will describe explicitly the structure the enhanced moduli possess. In what follows $\mathfrak{G}$ will be either of Lie algebras $\mathscr{L}^{m}$ or $\mathscr{L}^{\prime m}$, and $\hat{\mathfrak{G}}$ will be the central extension of $\left(\mathfrak{5}\right.$ by $\mathbb{C}$ as above. Put $\mathscr{L}_{00}:=t \mathbb{C}[[t]] \partial_{t} \subset \mathscr{L}_{0}=\mathbb{C}[[t]] \partial_{t} \subset \mathscr{L}, \mathscr{L}_{00}^{\prime}$ $=\mathscr{L}_{00}+\mathrm{gl}_{n}(\mathbb{C}[[t]]) \subset \mathscr{L}_{0}^{\prime}:=\mathscr{L}_{0}+\mathfrak{g l}_{n}(\mathbb{C}[[t]]) \subset \mathscr{L}^{\prime}, \quad \mathfrak{5}_{00}=\mathscr{L}_{00}^{(\prime) m} \subset \mathfrak{5}_{0}=\mathscr{L}_{0}^{(\prime) m} \subset(\mathfrak{5} ;$ these are "parabolic" subalgebras of $\mathfrak{5}$. It is easy to see that $\mathfrak{6}_{0}$ lifts uniquely to the subalgebra of $\hat{\mathfrak{5}}$; so we will assume that $\mathfrak{\mathfrak { F }}_{00} \subset \mathfrak{G}_{0} \subset \hat{\mathfrak{G}}$. The algebra $\mathfrak{G}_{00}$ is projective limit of finite dimensional Lie algebras. Moreover $\mathfrak{G}_{00}$ is Lie algebra of the proalgebraic group $K$, which is an extension of the finite dimensional reductive group $K^{r}$ by the prounipotent group $K^{u}$ (in case $\mathfrak{b}=\mathscr{L}$ one has $K=$ Aut $\mathbb{C}[[t]]$ $=$ group of formal diffeomorphisms of line $=\left\{a_{1} t+a_{2} t^{2}+\ldots, a_{i} \in \mathbb{C}, a_{1} \neq 0\right\}$, $K^{r}=\mathrm{G} m$; in case $\left(\mathfrak{g}=\mathscr{L}^{\prime}\right.$ one has $\left.K^{r}=\mathrm{G} m \times \mathrm{GL}_{n}\right)$. Clearly the adjoint action of $\mathfrak{F}_{00}$ on $(\mathfrak{5}$ integrates to the $K$-action.

Let $Y$ be a scheme. As usual, $(\mathfrak{6}, K)$-action on $Y$ consists of $\mathbf{5}$-action [i.e. Lie algebra map $(\mathfrak{H} \stackrel{\alpha}{\longrightarrow} \mathscr{T}(Y)]$ and $K$-action $K \times Y \rightarrow Y$ which are compatible in a sense that the $\mathbf{( 5}_{00}$-actions that come from $\left(\mathfrak{5}\right.$-action and $K$-action $\left(\boldsymbol{( \mathfrak { 5 }}_{00}=\right.$ Lie $\left.K\right)$ coincide, and for $k \in K, \tau \in \mathfrak{G}$ one has $\alpha(\operatorname{ad} k(\tau))=k^{*} \alpha(\tau)$. The action is (infinitesimally) transitive if for any $y \in Y$ the $\operatorname{map} \alpha_{y}:\left(\mathfrak{G} \rightarrow \mathscr{T}_{y}\right.$ is surjective; in this case we will call $Y$ a homogeneous $(\mathfrak{5}, K)$-scheme. The most important $(\mathfrak{b}, K)$-schemes are the following ones.

Definition 4.2.1. A $(\mathfrak{5}, K)$-action on $Y$ is of finite type if a sufficiently small "open" $K^{\prime} \subset K$ ("open" means "of finite codimension") acts on $Y$ in a free way with the quotient $K^{\prime} / Y$ being a scheme of finite type.

We will call such $Y$ 's $(\mathbf{5}, K)$-schemes of finite type for brevity (note that $Y$ itself is infinite dimensional!).

Similarly a $(\hat{\mathfrak{G}}, K)$-line bundle on a $(\mathfrak{6}, K)$-scheme $Y$ is just the line bundle $\lambda$ together with the compatible actions of $\hat{\mathfrak{F}}$ and $K$, that lift the $(\mathfrak{6}, K)$-action on $Y$. We will say that $\lambda$ has central charge $c$ if the element of the center $a \in \mathbb{C} \subset \hat{\mathfrak{G}}$ acts by multiplication by $c a$.

Clearly $\hat{\mathscr{M}}, \hat{\mathscr{M}}^{\prime}$ above are homogeneous $(\mathfrak{G}, K)$-schemes of finite type for the corresponding $\mathbf{6}$ 's and $\lambda, \lambda^{\prime}$ are $(\hat{\mathfrak{5}}, K)$-bundles of central charge 1 .

4.2.2. More General Example. Let $\hat{\mathscr{M}}_{g, 1 ; l, r}$ be the moduli space of data $(X, x, t$; $\left.y_{1}, \ldots, y_{l} ; u_{1}, \ldots, u_{l}\right)$, where $(X, x, t) \in \hat{\mathscr{M}}_{g, 1}, y_{i} \in X \backslash\{x\}$ are different points, and $u_{i}$ are $r$-jets of parameters at $y_{i}$. Then $(\mathscr{L}, K)$ acts on $\mathscr{M}_{g, 1 ; l, r}$ in the same manner as it did on $\hat{\mathscr{M}}_{g, 1} ; \hat{\mathscr{M}}_{g, 1 ; l, r}$ thus becomes a homogeneous $(\mathbf{5}, K)$-scheme of finite type, and the determinant bundle $\lambda$ also gets $(\hat{\mathscr{L}}, K)$-action of central charge 1 . Certainly, we 
may complicate the picture by switching on more $x$ 's together with a vector bundle.

4.2.3. For a $(\mathfrak{5}, K)$-scheme $Y$ and $p \in Y$ denote by $\left(\mathfrak{b}_{p}\right.$ the stabilizer of $p$; if $\lambda$ is a $(\hat{\mathfrak{G}}, K)$-bundle with central charge $\neq 0$, then $\hat{\mathfrak{G}}_{p}$ lifts to a subalgebra of $\hat{\mathfrak{G}}$. So for $Y=\hat{M}_{g, 1 ; l, r}$ and $p=\left(X, x, t, y_{i}, u_{i}\right)$ one has $\mathscr{L}_{p}=$ algebra of holomorphic vector fields on $X \backslash\{x\}$ with zero $r$-jets at $y_{i}$ (for example, in case $Y=\hat{\mathscr{M}}_{0,1 ; 1, r}$ the corresponding algebra is $\left\{L_{-r}, L_{-r-1}, \ldots\right\} \subset \mathscr{L}$, where $\left.L_{i}=t^{i+1} \partial_{t}\right)$. Call a subalgebra $Q \subset \hat{\mathfrak{G}}$ algebraic if there exists a homogeneous $(\mathfrak{5}, K)$-scheme $Y$ of finite type with $(\widetilde{\mathfrak{G}}, K)$-sheaf $\lambda$ of central charge $\neq 0$, and a point $p \in Y$ such that $Q=\mathfrak{G}_{p}$. Clearly, algebraic subalgebras are complementary to $\mathfrak{G}_{00}=\operatorname{Lie} K$ up to finite dimensional space [i.e. both $\operatorname{dim}\left(Q \cap\left(\mathfrak{5}_{00}\right), \operatorname{dim}\left(\mathbf{5} / Q+\mathfrak{5}_{00}\right)\right.$ are finite], and does not contain the center $\mathbb{C} \subset \mathbb{G}$. We do not know whether there exist a subalgebra of this type which is not algebraic.

\subsection{Relation with Sato Grassmannians}

The construction of 4.1 may be easily interpreted in the framework of Sato construction $[19,28]$. Consider the case, say, of $\hat{\mathscr{M}}_{g, 1}^{\prime}$ (the case of $m$ points is absolutely similar, the same as for the moduli of 4.2.2). The points of the Sato Grassmannian $G_{S}$ are the colattices $L \subset F=\mathbb{C}((t))^{n}$, i.e. the vector subspaces such that both $L \cap V_{0}, F / L+V_{0}$ are finite dimensional; here $V_{0}:=\mathbb{C}[[t]]^{n} \subset F$. Given a lattice $V \subset F$ (see 2.4.1) let $G_{S}^{(V)} \subset G_{S}$ be the set of $L$ 's transversal to $V$, i.e. $G_{S}^{(V)}:=\{L: L \oplus V \cong F\}$. Clearly, $G_{S}^{(V)}$ is $\operatorname{Hom}(F / V, V)$-torsor, hence a projective limit of finite-dimensional spaces. This defines on $G_{S}^{(V)}$ the structure of a projective limit of algebraic varieties; more precisely, $G_{S}^{(V)}$ is isomorphic to the spectrum of the polynomial ring of infinitely many variables. These structures for different $V$ 's glue together, hence $G_{S}$ becomes an (infinite dimensional) scheme. The Lie algebra $\mathscr{R} \subset$ End $F$ (see 2.4.1) naturally acts on $G_{S}$. Now we have a canonical map $\Phi: \hat{\mathscr{M}}^{\prime} \rightarrow G_{S}, \Phi(X, E, x, t, I):=$ image of $E(\dot{X}) \rightarrow E(\dot{U}) \stackrel{I^{-1}}{\longrightarrow} F$. It is easy to see that $\Phi$ is embedding of schemes equivariant with respect to the obvious map $\mathscr{L}^{\prime} \hookrightarrow \mathscr{R}$; so $\hat{\mathscr{M}}^{\prime}$ is the $\mathscr{L}^{\prime}$-orbit in $G_{S}$.

The scheme $G_{S}$ carries a canonical determinant sheaf $\Lambda$ with fiber $\Lambda_{L}$ at $L \in G_{S}$ equal to $\operatorname{det}\left(L \cap V_{0}\right) / \operatorname{det}\left(F / L+V_{0}\right)=\operatorname{det}\left(L \oplus V_{0} \rightarrow F\right)$; so $\left.A\right|_{\hat{M}^{\prime}}$ coincides with $\lambda^{\prime}$. The $\mathscr{R}$-action on $G_{S}$ extends naturally to $\widehat{\mathscr{R}}$ action on $\Lambda$ with central charge 1 (see [19]). Namely, to define the action of $r \in \widehat{\mathscr{R}}$ choose $(A, B) \in Q$ such that $r=(A, B) \bmod \mathscr{K}$, $A(F) \subset V_{0}$. For $L \in G_{S}$ choose a lattice $V \subset V_{0}$ such that $V \cap L=0, B(V)=0$, and a (finite dimensional) subspaces $T, T^{\prime} \subset F$ such that $V \oplus T=V_{0}^{n}, V \oplus T^{\prime} \oplus L=F$. Then for any $L^{\prime} \in G_{S}$ close to $L$ one has also the decomposition $V \oplus T^{\prime} \oplus L^{\prime} \cong F$, and so $\Lambda_{L^{\prime}}$ $=\operatorname{det}\left(V_{0} \rightarrow F / L^{\prime}\right)=\operatorname{det}\left(V_{0} / V\right) / \operatorname{det}\left(F / V+L^{\prime}\right)$ identifies with the constant line bundle $\operatorname{det} T / \operatorname{det} T^{\prime}$. Under this identification $r$ acts as a $1^{\text {st }}$ order differential operator $\delta(r)$ $+\operatorname{tr} A_{T T}-\operatorname{Tr}(A+B)_{T^{\prime} T^{\prime}}$. Here $\operatorname{tr} A_{T T} \in \mathbb{C}$ is the trace of $T T$-component of $A$ with respect to the decomposition $V_{0}=V+T$, and the function $\operatorname{tr}(A+B)_{T^{\prime} T^{\prime}}$ takes at $L^{\prime}$ the value equal to the trace of $T^{\prime} T^{\prime}$-component of $\delta(r)$ with respect to the decomposition $F=V \oplus T^{\prime} \oplus L^{\prime}$. Now one may see that the $\hat{\mathscr{L}}^{\prime}$-action on $\lambda^{\prime}$ comes from the $\hat{\mathscr{R}}$-action via the embedding $\hat{\mathscr{L}}^{\prime} \hookrightarrow \hat{\mathscr{R}}$ (see 2.4.2).

In the next few sections we will study certain distinguished $\mathscr{D}$-modules and local systems on moduli spaces. These $\mathscr{D}$-modules are just the equations on 
correlators in conformal 2D quantum field theory. The subject may be viewed as the first step in the study of lisse representations of Virasoro and current algebras. These representations are analogues of finite dimensional representations of finite dimensional Lie algebras (the term "lisse" is borrowed from a $\mathscr{D}$-module dictionary); in the Kac-Moody case "lisse" means "integrable."

What follows is a sketch of a part of a joint work with B. Feigin (in preparation); we omitted all lengthy proofs, as well as (the most) important part about the factorisation properties (degeneration near the boundary of moduli spaces). We would like to stress how much this material owes to V. Drinfeld. Above all it was his key theorem on holonomicity of the partition function (4.7.3) that was our starting point.

\subsection{From Representations to $\mathscr{D}$-Modules}

Assume that a Lie algebra $(\mathfrak{b}$ acts on $(Y, \mathscr{A})$, where $Y$ is an algebraic variety, and $\mathscr{A}$ is an Atiyah algebra on $Y$. This means that we have a Lie algebra map $(\mathfrak{H} \stackrel{\alpha}{\longrightarrow} \mathscr{A}(Y)$, hence the morphism of algebras $\alpha: \mathrm{U}(\mathfrak{G}) \rightarrow \mathscr{D}_{\mathscr{A}}(Y)$.

Remark. Any action of $(\mathfrak{H}$ on $(Y, \mathscr{A})$ defines a $(\mathfrak{5}$-action on $Y$, namely $(\mathfrak{5} \rightarrow \mathscr{A}(Y) \stackrel{\varepsilon}{\longrightarrow} \mathscr{T}(Y)$; any action of $\mathfrak{5}$ on $Y$ [i.e. Lie algebras map $\mathfrak{5} \rightarrow \mathscr{T}(Y)]$ defines the $\left(5\right.$-action on $\left(Y, \mathscr{A}_{\mathcal{O}_{Y}}\right)$.

Now the $(\mathfrak{G}$-action on $(Y, \mathscr{A})$ defines the localisation functor $\Delta:(\mathfrak{5}$-modules $\rightarrow \mathscr{D}_{\mathscr{A}}$-modules by formula $\Delta(M):=\mathscr{D}_{\mathscr{A}} \underset{\mathrm{U}(\mathfrak{G})}{\otimes} M$. Clearly $\Delta$ is right exact.

Example. If $M$ is generated by a single element $\varphi$ subject to relations $A_{i} \varphi=0$, $A_{i} \in \mathrm{U}(\mathfrak{G})$, then $\Delta(M)$ is a $\mathscr{D}_{\mathscr{A}}$-module that represents a system of differential equations $\alpha\left(A_{i}\right) \varphi=0$.

Remark. The functor $\Delta$ when $(\mathfrak{5}$ is a finite dimensional reductive Lie algebra and $Y$ is a flag space of $(\mathfrak{5}$ appeared to be very convenient in representation theory (see e.g. [3]).

We will apply the above construction to the situation of 4.2. Namely, let $Y$ be any $((\mathfrak{5}, K)$-scheme of finite type and $\lambda$ be a $(\hat{\mathfrak{G}}, K)$-bundle on $Y$ with central charge $c^{\prime} \neq 0$. Put $\mathscr{A}=\mathscr{A}_{c}=c / c^{\prime} \mathscr{A}_{\lambda}, \mathscr{D}_{\mathscr{A}}=\mathscr{D}_{c}$ (here $c \in \mathbb{C}$ is a fixed constant). Since the dimensions of our varieties are infinite one has to worry a little and consider the continuous modules only. A $\hat{\mathfrak{G}}$-module $M$ is continuous iff the stabilizer of any $z \in M$ is an "open" subalgebra in $\hat{\mathfrak{G}}$; a $\mathscr{D}_{c}$-module is continuous iff any of its local section is $K^{\prime}$-invariant for a sufficiently small "open" subgroup $K^{\prime} \subset K$. Denote by $\mathfrak{G}_{c}$-mod the category of continuous $\left(\mathfrak{5}\right.$-modules of central charge $c$, and by $\mathscr{D}_{c}$-mod the one of continuous $\mathscr{D}_{c}$-modules. We get the functor $\Delta: \mathfrak{G}_{c}$-mod $\rightarrow \mathscr{D}_{c}$-mod.

Let $p \in Y$ be a point, $\hat{\mathfrak{b}}_{p} \subset \hat{\mathfrak{G}}$ be its stabiliser, and $m_{p} \subset \mathcal{O}_{p}$ be its maximal ideal. One has the simple

4.4.1. Lemma. $M / \mathfrak{G}_{p} M=\Delta(M) / \mathrm{m}_{p} \Delta(M)$ (i.e. the fiber at $p$ of $\Delta(M)$ as a $\mathcal{O}_{p}$-module coincides with the coinvariants of $M$ with respect to $\left(\mathfrak{b}_{p}\right)$.

\subsection{Descent to Finite Dimensional Quotients}

Let $K^{\prime} \subset K$ be an "open" subgroup that acts on $Y$ in a free way, and $\pi_{K^{\prime}}: Y \rightarrow K^{\prime} \backslash Y$ be the projection (so $K^{\prime} \backslash Y$ is a finite dimensional variety). The sheaf $\lambda$ descends to the 
same-noted sheaf on $K^{\prime} \backslash Y$, hence we have the category $\mathscr{D}_{c}$ - $\bmod \left(K^{\prime} \backslash Y\right)$ of $\mathscr{D}_{c}$-modules on $K^{\prime} \backslash Y$ together with inverse image functor $\pi_{K^{\prime}}^{+}: \mathscr{D}_{c}$-mod$\left(K^{\prime} \backslash Y\right)$ $\rightarrow \mathscr{D}_{c}-\bmod \left(:=\mathscr{D}_{c}-\bmod (Y)\right), \pi^{+}(R)=\mathscr{O}_{Y} \otimes R$. In fact, $\pi^{+}$defines equivalence between $\mathscr{D}_{c}-\bmod \left(K^{\prime} \backslash Y\right)$ and the category $\left(\mathscr{D}_{c}, K^{\prime}\right)$-mod of $K^{\prime}$-equivariant $\mathscr{D}_{c}$-modules on $Y$ (here a $K^{\prime}$-equivariant $\mathscr{D}_{c}$-module is a $\mathscr{D}_{c}$-module with $K^{\prime}$-action such that the corresponding Lie $K^{\prime}$-action coincides with the one induced by Lie $K^{\prime} \subset\left(\mathfrak{S} \subset \mathscr{D}_{c}\right)$.

4.5.1. Remarks. a) If $K^{\prime}$ does not acts freely, then $K^{\prime} \backslash Y$ is a stack, and, being defined in an appropriate way, a $\mathscr{D}_{c}$-module on $K^{\prime} \backslash Y$ is again the same as a $\left(\mathscr{D}_{c}, K^{\prime}\right)$ module on $Y$. b) In fact, the descent of $\mathscr{D}$-modules usually does not require the group action. Namely, if $S, T$ are smooth varieties and $\pi: S \rightarrow T$ is a smooth morphism with connected fibers, then $\pi^{+}: \mathscr{D}_{T}-\bmod \rightarrow \mathscr{D}_{S}$-mod is a fully faithful embedding, $\pi^{+}\left(\mathscr{D}_{T}\right.$-mod) consists of $\mathscr{D}_{S}$-modules generated by sections horizontal along the fibres of $\pi$. This remark implies, in particular, that any finitely generated continuous $\mathscr{D}_{c}$-module on $Y$ comes from a certain (finite dimensional) quotient $K^{\prime} \backslash Y$.

Below we will confine ourselves with the pure Virasoro case $\hat{\mathfrak{G}}=\hat{\mathscr{L}}^{m}$. Our $\hat{\mathfrak{G}}$-modules $M$ 's will be integrable with respect to $\mathfrak{G}_{00}$ (i.e. for any $z \in M$ one should have $\operatorname{dim}\left(\mathfrak{F}_{00} z<\infty\right)$. This implies that $M$ is naturally a $\left(\mathfrak{G}, K^{u}\right)$-module, hence $\Delta(M)$ is a $\left(\mathscr{D}_{c}, K^{u}\right)$-module, so $\Delta(M)$ descends to (the same noted) $\mathscr{D}_{c}$-module on $K^{u} \backslash Y$; if $M$ was finitely generated, then $\Delta(M)$ is coherent. Moreover, we have $K^{r}=K / K^{u}$ $=\mathrm{Gm}^{m}$, and $\Delta(M)$ is $K^{r}$-monodromic along the fibers of projection $K^{u} \backslash Y \rightarrow K \backslash Y$. Note that in case $Y=\hat{\mathscr{M}}_{g, m}$ one has $K^{u} \backslash Y=\hat{\mathscr{M}}_{g, m ; 1}, K \backslash Y=\mathscr{M}_{g, m}$, hence we get (twisted) $\mathscr{D}$-modules on moduli spaces.

4.5.2. Examples. Assume that $m=1$. Let $M_{c, h}$ be a Verma module: it is an $\hat{\mathscr{L}}_{c}$-module generated by $v \in M_{c, h}$ subject to relations $L_{i} v=0$ for $i>0, L_{0} v=h v$ (here $\left.L_{i}:=t^{i+1} \partial_{t} \in \hat{\mathscr{L}}_{c}\right)$. Then $\Delta\left(M_{c, h}\right)$ as $\mathscr{D}_{c}$-module on $\mathscr{M}_{g, 1 ; 1}$ is generated by the single section $v$ subject to the relation $L_{0} v=h v$ (note that $L_{0}$ is a Euler vector field along the fibers of $\left.\mathscr{M}_{g, 1 ; 1} \rightarrow \mathscr{M}_{g, 1}\right)$. In particular $\Delta\left(M_{c, 0}\right)$ descends to the free $\mathscr{D}_{c}$-module on $\mathscr{M}_{g, 1}$. Let $\bar{M}_{c, 0}$ be the quotient of $M_{c, 0}$ by the submodule generated by $L_{-1} v$ (hence $\bar{M}_{c, 0}$ is an $\hat{\mathscr{L}}$-module induced by the trivial representation of $\left(\mathfrak{G}_{0}\right)$. Then 4.5.1. b implies that $\Delta\left(\bar{M}_{c, 0}\right)$ descends to the free $\mathscr{D}_{c}$-module on $\mathscr{M}_{g}$.

\subsection{Lisse Modules}

The irreducible $\hat{\mathscr{L}}_{c}$-modules $L_{c, h}$ are just the quotients of Verma modules by maximal submodules: $L_{c, h}=M_{c, h} / N_{c, h}$; the irreducible $\hat{\mathscr{L}}_{c}^{m}$-modules are tensor products $L_{c, h}=L_{c, h_{1}} \otimes L_{c, h_{2}} \otimes \ldots \otimes L_{c, h_{m}}$. We will be concerned with the following:

Problem. When $\Delta\left(L_{c, h}\right)$ is lisse, i.e. is a vector bundle with $\lambda^{c}$-twisted integrable connection?

4.6.1. First one should chat about singular supports. Let $M$ be a finitely generated ( $\mathscr{L}_{00}$-integrable) $\hat{\mathscr{L}}_{c}$-module. One defines its singular support $\mathrm{SS}(M) \subset \mathscr{L}^{*}$ just as in the usual finite dimensional situation. Namely, take $R \subset M$ to be a finite dimensional subspace that generates $M$; it defines the filtration $M_{i}=\mathrm{U}_{i} R$ on $M$ $\left[\right.$ here $\left.\mathrm{U}_{i}=\mathbb{C}+\hat{\mathscr{L}}+\ldots+\hat{\mathscr{L}}^{i} \subset \mathrm{U}_{c}(\hat{\mathscr{L}})\right]$. We get a $S^{\prime}(\mathscr{L})$-module Gr. $M=\oplus M_{i} / M_{i-1}$ 
[since $\mathrm{U}_{i} / \mathrm{U}_{i-1}=S^{i}(\mathscr{L})$ ], generated by $R=\operatorname{Gr}_{0}(M)$. Put $\operatorname{SS}(M) \subset \mathscr{L}^{*}=\operatorname{Spec} S^{*}(\mathscr{L})$ as the support of Gr. $(M)$; this definition does not depend on the choice of $R$. Clearly $\operatorname{SS}(M) \subset \mathscr{L}_{00}^{\frac{1}{1}}$; it is Zariski closed and ad $\mathscr{L}_{00}$-invariant. An easy linear algebra exercise shows that these properties imply that $\operatorname{SS}(M)$ is either 0 or coincides with one of the vector subspaces $\mathscr{P}_{i}^{\perp} \subset \mathscr{L}^{*}$, where $\mathscr{P}_{i}=\left\{L_{i}, L_{i+1}, \ldots\right\}, i \leqq 0$ (so $\mathscr{P}_{0}=\mathscr{L}_{00}, \mathscr{P}_{-1}=\mathscr{L}_{0}$ ).

4.6.2. Examples. If $M$ is generated by a single vector $v$, then $\operatorname{SS}(M)$ is given by equations $\operatorname{Symb}(P)=0, P \in \operatorname{Ann}(M, v)$; here $\operatorname{Ann}(M, v)=\left\{P \in \mathrm{U}_{c}(\hat{\mathscr{L}}): P v=0\right\}$, and Symb is a higher order term. Hence $\operatorname{SS}\left(M_{c, h}\right)=\mathscr{L}_{00}^{\perp}, \operatorname{SS}\left(\bar{M}_{c, 0}\right)=\mathscr{L}_{0}^{\perp}$. More generally, let $N \subset M_{c, h}$ be a submodule generated by one singular vector $v_{N}\left(L_{i} v_{N}=0\right.$ for $i>0)$. Then $\operatorname{SS}\left(M_{c, h} / N\right)=\mathscr{L}_{0}^{\perp}$. This follows from the fact (communicated to us by D. B. Fuchs) that $v_{N}=P_{N} v$ with $\operatorname{Symb}\left(P_{N}\right)$ equals the power of $L_{-1}$ (see [12]).

4.6.3. Definition. Call $M$ lisse if $\mathrm{SS}(M)$ does not equal $\mathscr{L}_{00}^{\perp}$ or $\mathscr{L}_{0}^{\perp}$ (i.e. if $\left.\mathrm{SS}(M) \subset \mathscr{P}_{-2}^{\perp}\right)$.

Commentary. An infinite dimensional version of Gabber's integrability theorem [30] would imply $\mathrm{SS}(M)$, being a vector space, should be an orthogonal complement to the Lie subalgebra of $\mathscr{L}$. Hence "lisse" above should really mean that $\operatorname{SS}(M)=0$, which explains the term. It seems that it is really a fact, but we could not prove it.

4.6.4. Remark. Consider the irreducible module $L_{c, h}=M_{c, h} / N_{c, h}$. Then $L_{c, h}$ is lisse iff the following condition holds: there exists $P\left(L_{-1}, L_{-2}, \ldots\right)$ such that $P v \in N_{c, h}$ [i.e. $\left.P \in \operatorname{Ann}\left(L_{c, h} v\right)\right]$ and $\operatorname{Symb}(P)$ is prime to $L_{-1}$.

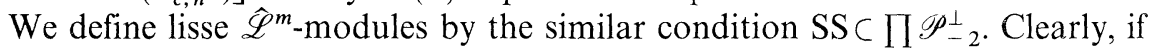
$M_{1}, \ldots, M_{m}$ are lisse $\hat{\mathscr{L}}$-modules, then $M_{1} \otimes \ldots \otimes M_{m}$ is a lisse $\hat{\mathscr{L}}^{m}$-module, and the converse is also true.

4.6.5. Theorem. Assume that our $Y$ is homogeneous. If $M$ is a lisse $\hat{\mathscr{L}}_{c}^{m}$-module, then $\Delta(M)$ is lisse.

Sketch of the Proof. Consider the case $m=1$; the general case is similar. We will show that the singular support of $\Delta(M)$ coincides with the zero section of the cotangent bundle $\Omega(Y)$. Look at the moment map for the $\mathscr{L}$-action $\mu: \Omega(Y) \rightarrow \mathscr{L}^{*}$. It is easy to see that $\mathrm{SS}(\Delta(M)) \subset \mu^{-1} \mathrm{SS}(M) \subset \mu^{-1}\left(\mathscr{P}_{-2}^{\perp}\right)$. But the maximal involutive subvariety of $\mu^{-1}\left(\mathscr{P}_{-2}^{\perp}\right)$ is zero, since $\mathscr{P}_{-2}$ generates $\mathscr{L}$ and the $\mathscr{L}$-action is infinitesimally transitive. This implies that $\mathrm{SS}(\Delta(M))=0$ due to the usual integrability theorem for SS of $\mathscr{D}$-modules; note that $\Delta(M)$ comes from a finitedimensional quotient, hence the situation is actually finite-dimensional.

4.6.6. Corollary. Let $Q \subset(5$ be an algebraic subalgebra (see 4.2.3) and $M$ be a lisse (5-module. Then $\operatorname{dim} M / Q M<\infty$.

The proof follows directly from 4.6.4 and 4.4.1.

\subsection{Classification of Lisse Modules}

As follows from, say, [12] the maximal submodules $N_{c, h}$ of Verma modules $M_{c, h}$ are either 0 , or generated by one or two singular vectors. One has also the following lemma, well known to specialists on Virasoro algebra (see [6]). 
4.7.1. Lemma. $N_{c, 0}$ is generated by two singular vectors iff $c=c_{p, q}=1$ $-6(p / q+q / p-2)$ for certain rational prime integers $p, q, 1<p<q$.

4.7.2. Lemma. $L_{c, 0}$ is lisse iff $c$ is satisfies the conditions of 4.7.1.

Proof. If $N_{c, 0}$ is generated by one singular vector, then $\operatorname{SS}\left(L_{c, 0}\right)=\mathscr{L}_{0}^{\perp}$ (4.6.2). Assume that $N_{c, 0}$ is generated by two singular vectors (one of them is $L_{-1} v$ ). Take $P\left(L_{-1}, L_{-2}, \ldots\right)$ such that $P \cdot v \in N_{c, 0} \backslash \mathrm{U}(\hat{\mathscr{L}}) L_{-1} v$ of minimal degree. Then $\operatorname{Symb}(P)$ is prime to $L_{-1}$ [otherwise take $R \in \mathrm{U}(\hat{\mathscr{L}})$ with $\operatorname{Symb} P=(\operatorname{Symb} R) \cdot L_{-1}$; we have $\left(P-R L_{-1}\right) v \in N_{c, 0} \backslash \mathrm{U}(\hat{\mathscr{L}}) L_{-1} v$ and $\left.\operatorname{deg}\left(P-R L_{-1}\right)<\operatorname{deg} P\right]$, and we may apply 4.6.4.

4.7.3. Corollary. If $c=c_{p, q}$, then $\Delta\left(L_{c, 0}\right)$ is a vector bundle with a $\lambda^{c}$-twisted connection on the moduli space of curves $\mathscr{M}_{g}$.

The $\mathscr{D}_{c}$-module $\Delta\left(L_{c_{p, q}, 0}\right)$ is just an equation on the partition function in the minimal theory of central charge $c_{p, q},[6]$.

4.7.4. Now for $c=c_{p, q}$ consider the following list of $h$ 's:

$$
h_{m, n}=\frac{1}{4}\left[2-\frac{p}{q}-\frac{q}{p}-\frac{q}{p}\left(n-\frac{p}{q} m\right)^{2}\right],
$$

where $1 \leqq m \leqq q-1,1 \leqq n \leqq p-1$.
Clearly $h_{11}=0$, one has $h_{m, n}=h_{q-m, p-n}$, and the list contains $\frac{(p-1)(q-1)}{2}$ different $h$ 's.

4.7.5. Theorem. a) The following conditions for an irreducible module $L_{c, h}$ are equivalent:

(i) $L_{c, h}$ is lisse.

(ii) $N_{c, h}$ is generated by two singular vectors and occurs in $M_{c, h}$ only (i.e. no other $M_{c, h}$, contains $L_{c, h}$ as a subquotient).

(iii) The invariant space $L_{c, h}^{\left(L_{3}, L_{4}, \ldots\right)}$ is finite dimensional.

(iv) $c=c_{p, q}$ from 4.7.1 and $h=h_{m n}$ from 4.7.4.

b) Any lisse $\hat{\mathscr{L}}_{c}^{m}$-module is a finite direct sum of tensor products of lisse irreducible $\hat{\mathscr{L}}_{c}$-modules.

\subsection{Local Systems on Moduli Spaces}

Let us consider the case $Y=\hat{M}_{g m}$. One has the following:

4.8.1. Lemma. The $\mathscr{D}_{c}$-module $\Delta\left(L_{c, h_{1}} \otimes \ldots \otimes L_{c, h_{m}}\right)$ equals 0 if among $L_{c, h}$ 's both lisse and non-lisse irreducibles occur.

It readily implies

4.8.2. Corollary. Consider the obvious projection $\pi: \hat{\mathscr{M}}_{g, m_{1}+m_{2}} \rightarrow \hat{\mathscr{M}}_{g m_{1}}$. Let $L_{c, h_{i}}$, $i=1, \ldots, m_{1}$, be lisse $\hat{\mathscr{L}}_{c}$-irreducibles. Then we have the canonical isomorphism

$$
\pi^{+}\left(\Delta\left(L_{c, h_{1}} \otimes \ldots \otimes L_{c, h_{m_{1}}}\right)\right)=\Delta\left(L_{c, h_{1}} \otimes \ldots \otimes L_{c, h_{m_{1}}} \otimes L_{c, 0} \otimes \ldots \otimes L_{c, 0}\right) .
$$

So we may insert the $L$ 's with $h=0$ at any additional points, and the coinvariants - the fibers of the corresponding $\mathscr{D}_{c}$-modules - will not change. 
4.8.3. Now - for a moment - let us fix a point $p=\left(X, x_{i}, t_{i}\right) \in \hat{\mathscr{M}}_{g, m}$. The fiber $\Delta\left(L_{c, h_{1}} \otimes \ldots \otimes L_{c, h_{m}}\right)_{p}$ is the space of coinvariants of $\mathscr{T}\left(X \backslash\left\{x_{i}\right\}\right) \subset \hat{\mathscr{L}}_{c}^{m} ;$ such things are familiar in automorphic representation theory (the automorphic representations are representations of "adelic groups," i.e. the product of "local" ones having the vector or functional fixed under the "global" group). This analogy was also noticed by Witten [29]. One may exploit this in a slightly different direction to construct the "automorphic proliferation" functor that replaces tensor products in a finite dimensional situation.

Namely, assume that $\left\{x_{i}\right\}=S \cup S^{\prime}, S \cap S^{\prime}=\emptyset$; let $\hat{\mathscr{L}}_{S}, \hat{\mathscr{L}}_{S^{\prime}}$ be the products of corresponding Virasoro algebras (so $\hat{\mathscr{L}}^{m}$ is $\hat{\mathscr{L}}_{S} \times \hat{\mathscr{L}}_{S^{\prime}}$ with centers identified); let $\tilde{\mathscr{T}}=\tilde{\mathscr{T}}\left(X \backslash\left\{x_{i}\right\}\right) \hookrightarrow \hat{\mathscr{L}}_{S} \times \hat{\mathscr{L}}_{S^{\prime}}$ be the subalgebra of the global vector field (extended by a 1 -dimensional center). Clearly both projections

$$
\tilde{\mathscr{T}} \stackrel{v_{S}}{\longrightarrow} \hat{\mathscr{L}}_{S}, \tilde{\mathscr{T}} \stackrel{v^{\prime}}{\longrightarrow} \hat{\mathscr{L}}_{S^{\prime}}
$$

have dense image. One has the adjoint functors

$$
\tilde{\mathscr{T}} \text {-mod } \stackrel{v_{S}^{+}}{v_{S_{+}+}^{\longleftrightarrow}} \hat{\mathscr{L}}_{S^{-} \text {mod }}
$$

(here $\tilde{\mathscr{T}}$-modules are arbitrary ones, and $\hat{\mathscr{L}}_{S}$-modules are $\hat{\mathscr{L}}_{S 00}$-integrable) $v_{S}^{+}$is just the restriction, and $v_{S+}(M)$ is the largest submodule of $M$ on which $\tilde{\mathscr{T}}$-action

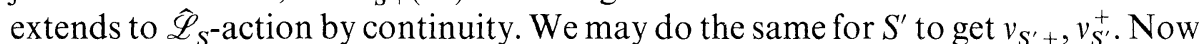
define the proliferation functor $P_{S^{\prime}, S}: \hat{\mathscr{L}}_{S^{-}} \bmod \rightarrow \hat{\mathscr{L}}_{S^{\prime}}-\bmod$ by formula $P_{S^{\prime}, S}(M)$ $=v_{S^{\prime}+} \mathrm{D} v_{S}^{+} M$; here $\mathrm{D}$ means dual $\mathscr{T}^{\prime}$-representation (arbitrary linear functionals). Clearly $M \otimes P_{S^{\prime} S}(M)$ carries canonical (universal) $\tilde{\mathscr{T}}$-invariant linear form.

The above results imply

4.8.4. Lemma. $P_{S^{\prime} . S}$ transforms lisse modules to lisse ones.

Example. Let $X=\mathbb{P}^{1}$. If $S=\{0\}, S^{\prime}=\{\infty\}$, then $P_{\mathrm{SS}^{\prime}}(M)$ is a contragradient module; if $M$ is irreducible, then $P_{\mathrm{SS}}(M)$ is isomorphic to $M$. If $S=\{0\}, S^{\prime}=\{1, \infty\}$, then $P_{\mathrm{SS}^{\prime}}$ may be described explicitly (for lisse modules). In particular $P_{\mathrm{SS}^{\prime}}\left(L_{c, 0}\right)$ $=\oplus L_{h_{m n}} \otimes L_{h_{m n}}$, the sum runs through $\frac{(p-1)(q-1)}{2}$-representations of list 4.7.4.

The bundles on $\mathscr{M}_{0,3}$ that correspond to irreducible lisse $\hat{\mathscr{L}}^{3}$-modules are either 1 dimensional, or 0 ones.

4.8.5. So for any lisse $\hat{\mathscr{L}}_{c}^{m}$-module $M$ we get a lisse $\mathscr{D}_{c}$-module on $\mathscr{M}_{g m ; 1}$. As usual, we may consider the sheaves of their horizontal sections (or solutions). These are local systems on the fibration $\lambda^{\cdot}$ over $\mathscr{M}_{g, m ; 1}$, where $\lambda^{*}$ is $\lambda$ with zero section removed, that have monodromy $\exp (2 \pi i c)$ along the fibers of $\lambda$. Equivalently, these are representations of $\pi_{1}\left(\lambda^{\circ}\right)$, which is a central extension of the Teichmuller group $\pi_{1}\left(\mathscr{M}_{g, m ; 1}\right)$ by $\mathbb{Z}$; the generator of the center acts by $\exp (2 \pi i c)$. It would be very interesting to find these representations explicitly; the specialists seem to know only the cases of $\mathscr{M}_{0,4 ; 1}$ and $\mathscr{M}_{1,0 ; 1}$. The only thing we have at the moment is a very plausible conjecture on their ranks. It is closely related to the description of these local systems near the boundary of moduli space, and it is here that what physicists call "operator algebra" comes into play. We refer the reader to the above mentioned paper in preparation on these subjects. 


\section{5. $C^{\infty}$-Connections on Determinant Bundles}

In this section we will consider the analytic situation. We will see that 2.3 helps to construct a canonical $\mathrm{C}^{\infty}$-connection $\nabla_{\lambda}$ on $\lambda_{E}$ from $\mathrm{C}^{\infty}$-connections $\nabla_{E}$ on $E$ and $\nabla_{\omega}$ on $\omega$, that has the curvature prescribed by the Riemann-Roch-Grothendieck formula. This construction is, in a sense, the once integrated version of a theorem by Quillen, Belavin-Khizhnik, Bost [18, 5, 9], generalised recently by BismutGillet-Soule [7] to higher dimensions. To find the exact formula for "det $\overline{\partial "}$ " one has to integrate twice ....

In what follows $\Omega_{X}^{00} \stackrel{\bar{D}}{\longrightarrow} \Omega_{X}^{01} \stackrel{\bar{o}}{\longrightarrow} \ldots$ will be the $\bar{\partial}$-resolution of $\mathcal{O}_{X}$, so $\Omega_{X}^{00}=\mathscr{C}_{X}$ is the sheaf of $C^{\infty}$-functions, and $\Omega_{X}^{0 q}$ is the sheaf of $(0, q)$-forms; the bicomplex $\Omega_{X}^{p q}$ $=\Omega^{p} \otimes_{\mathbb{O}_{X}} \Omega^{0 q}$ has differentials $\partial, \bar{\partial}$ and the total differential $\partial+(-1)^{p} \bar{\partial}$. These are (super) commutative dg-algebras. In the relative situation we may consider the relative forms $\Omega_{X / S}^{0 q}$ which form a $\bar{\partial}$-resolution of $\mathcal{O}_{X} \otimes_{\mathscr{U}_{S}} \pi^{-1} \mathscr{C}_{S}$, and $\Omega_{X / S}^{p, q}$ $=\Omega_{X / S}^{p} \otimes \Omega_{X / S}^{0 q}$.

\section{1.}

Let $\mathscr{A}$ be an $R$-Atiyah algebra on $X$. Put $\mathscr{C} R:=\mathscr{C}_{X} \otimes_{\mathscr{O}_{X}} R$ and denote by $\mathscr{C} \mathscr{A}$ the $\mathscr{C} R$-Atiyah algebra which is the push-forward of $\mathscr{A}$ [see 1.1.5(i)] by the map $R \rightarrow \mathscr{C} \otimes R$ and the action $\tau(f \otimes r)=\varepsilon(\tau)(f) \otimes r+f \otimes[\tau, r]$ of $\mathscr{A}$ on $\mathscr{C} R$. We have the exact sequence of sheaves

$$
0 \rightarrow \mathscr{A} \rightarrow \mathscr{C} \mathscr{A} \stackrel{\bar{\partial}}{\longrightarrow} \Omega^{01} \otimes R \stackrel{\bar{\partial}}{\longrightarrow} \ldots
$$

$A \mathrm{C}^{\infty}$-connection on $\mathscr{A}$ is just a connection $\nabla$ on $\mathscr{C} \mathscr{A}$. Clearly, $\mathrm{C}^{\infty}$-connections form $\Omega^{10} \otimes R$-torsor which has global sections. The $\mathrm{C}^{\infty}$-curvature of $\nabla$ is $\mathrm{C}_{V}=\mathrm{C}_{V}^{11}$ $+\mathrm{C}_{\nabla}^{20} \in \Omega_{X}^{11} \otimes R \oplus \Omega^{20} \otimes R$, where $\mathrm{C}^{20}$ is the usual curvature (see 1.1.4), $\mathrm{C}^{20}\left(\tau_{1}, \tau_{2}\right)$ $=\left[\nabla\left(\tau_{1}\right), \nabla\left(\tau_{2}\right)\right]-\nabla\left(\left[\tau_{1}, \tau_{2}\right]\right), \tau \in \mathscr{T}_{X}$, and $\mathrm{C}^{11}:=\bar{\partial} \nabla, \mathrm{C}_{\nabla}^{11}(\bar{\tau}, \tau)=\bar{\tau}(\nabla(\tau))$. We will say that $\nabla$ is a $(1,1)$-connection if $\mathrm{C}_{\nabla}^{20}=0$.

\section{2 .}

Assume that we are in a relative situation of 1.2. Let $\mathscr{A}$ be a $\pi$-algebra. The algebra $\mathscr{T}_{\pi}$, hence $\mathscr{A}$, acts on the complex $\Omega_{\dot{X} \mid S}^{*}$ by the formulas $\tau(\omega):=\operatorname{Lie}_{\tau}(\omega), \chi(\omega)$ $=\chi\lrcorner \omega, \tau \in \mathscr{T}_{\pi}^{0}, \chi \in \mathscr{T}_{\pi}^{-1}, \omega \in \Omega_{\dot{X} \mid S}^{*}$. Define the dg-Lie superalgebra $\mathscr{C} \mathscr{A}^{-}$to be the push-forward of $\mathscr{A}^{*}$ by the map $\Omega_{X / S} \hookrightarrow \Omega_{\dot{X} / S}^{*}$ and the above action. Hence $\mathscr{C} \mathscr{A}$ is an extension of $\mathscr{A}^{*} / \Omega_{X / S}[2]$ by $\Omega_{\dot{X} / S}^{*}[2]$. Clearly, $\pi(\mathscr{C} \mathscr{A}):=R^{0} \pi_{*} \mathscr{C} \mathscr{A}^{*}$ has a natural structure of a $\mathscr{C}_{S}$-Atiyah algebra, and we have the canonical isomorphism $\mathscr{C} \pi(\mathscr{A})$ $=\pi(\mathscr{C} \mathscr{A})$.

A $\pi^{-1} \mathcal{O}_{S^{-}}$linear section $\tilde{\nabla}: \mathscr{A} / \Omega_{X / S}[2] \rightarrow \mathscr{C} \mathscr{A}$ which commutes with the differential will be called a $\mathrm{C}^{\infty}$-connection on $\mathscr{A}$. Such $\tilde{\nabla}$ determines a $\mathrm{C}^{\infty}$ connection $\pi(\tilde{\nabla}):=R^{0} \pi_{*}(\tilde{\nabla})$ on $\pi\left(\mathscr{A}^{*}\right)$.

\subsection{Formal Parametrix}

Let us return to ${ }^{\text {tr }} \mathscr{A}_{E}$. Let $\nabla_{E}$ be a $\mathrm{C}^{\infty}$-connection on $E$ (i.e. a connection on $\mathscr{A}_{E}$ ) and $\nabla_{\omega}$ be a connection on $\omega$. We are going to construct the $\mathrm{C}^{\infty}$-connection $\tilde{\nabla}=\tilde{\nabla}_{\nabla_{E}, \nabla_{\omega}}$ on $\mathscr{C}^{\mathrm{tr}} \mathscr{A}_{E}$. This will be done using the formal parametrix. 

5.3.1. Let $F$ be a vector bundle. Put $\mathscr{C} \hat{\otimes} F:=\lim _{i} \mathscr{C} \bigotimes F / \mathscr{C} \bigotimes F(-i \Delta)$; hence the
elements of $\mathscr{C} \otimes \hat{\mathcal{O}}_{X}$ are formal series

$$
\sum_{i \geqq 0} f_{i}\left(t_{1}, \bar{t}_{1}\right)\left(t_{2}-t_{1}\right)^{i}, f_{i}(t, \bar{t}) \in \mathscr{C}
$$

A $C^{\infty}$-connection $\nabla$ on $F$ canonically defines the derivative $\nabla^{(2)}: \mathscr{C} \hat{\otimes} F \rightarrow \mathscr{C} \bowtie F \omega$ along the second variable: in coordinates, if $\nabla=\partial_{t}+A(t, \bar{t})$, then $\nabla^{(2)}=\partial_{t_{2}}$ $+\sum \partial_{t_{1}}^{i} A\left(t_{1}, \bar{t}_{1}\right) \times\left(t_{2}-t_{1}\right)^{i} / i$ !. If $G$ is another bundle, then $\nabla^{(2)}$ also acts on $\mathscr{C} G \hat{\otimes} \hat{F}$ $=G \otimes(\mathscr{C} \hat{\otimes} F)$. Now we may define $\operatorname{Id}_{E, \nabla_{E}} \in \mathscr{C} E \hat{\otimes} E^{*}$ to be the (only) $\nabla_{E^{*}}^{(2)}$-horizontal section that equals id ${ }_{E}$ being restricted to $\Delta$ (here $\nabla_{E^{*}}={ }^{t} \nabla_{E}$ ). In the same way we have $\nabla_{\omega}^{(2)}: \mathscr{C} \hat{凶} \omega \rightarrow \mathscr{C} \hat{凶} \omega^{\otimes 2}$, and, localising off $\Delta, \nabla_{\omega}^{(2)}: \mathscr{C} \mathscr{P} \rightarrow \mathscr{C} \mathscr{P} \omega$, where

$$
\begin{aligned}
\mathscr{C} \mathscr{P}:= & \mathscr{C} \hat{\otimes} \omega(\infty \Delta)=\overleftarrow{i}_{i}^{\lim } \mathscr{C} \bigotimes \omega(\infty \Delta) / \mathscr{C} \bigotimes \omega(-i \Delta), \\
& \mathscr{C} \mathscr{P} \omega=\mathscr{C} \mathscr{P} \otimes_{\mathbb{O}_{X}} \omega=\mathscr{C} \hat{\otimes} \omega^{\otimes 2}(\infty \Delta) .
\end{aligned}
$$

Define $\Pi_{\nabla_{\omega}} \in \mathscr{C} \mathscr{P}$ to be the (unique) solution of the equation $\nabla_{\omega}^{(2)}\left(\Pi_{\nabla_{\omega}}\right)+\Pi_{\nabla_{\omega}}^{2}$ $=0$ with a pole at $\Delta$. Equivalently, if $v \in \operatorname{Ker} \nabla_{\omega}^{(2)}, v \neq 0$, then $\Pi_{\nabla_{\omega}}=v / \int_{t_{1}}^{t_{2}} v$; here $\int_{t_{1}}^{t_{2}} v$ is the solution of equation $d^{(2)} \int v=v$ that equals 0 at $\Delta$. Finally, put $\Pi_{1}^{t_{1}}:=\Pi_{\nabla_{\omega}} \cdot \operatorname{Id}_{E, \nabla_{E}} \in \mathscr{C} \mathscr{P}_{E, 1}:=\mathscr{C} E \bigotimes E^{0}(\Delta)$. This $\Pi$ is called the formal parametrix for $\nabla_{E}, \nabla_{\omega}$; we have $\delta(\Pi)=1 \in \mathscr{D}_{E} \subset \mathscr{C} \otimes \mathscr{D}_{E}$, so $\Pi$ defines a section $s_{\Pi}$ of $\delta$, $s_{I I}(\partial)=\partial^{(1)} \Pi$ (derivative along the first variable).

5.3.2. Let us describe $s_{I I}$ more explicitly. Consider the expression

$$
P(x, y)=\left(\ldots+\frac{A_{-2}(x)}{(y-x)^{3}}+\frac{A_{-1}(x)}{(y-x)^{2}}+\frac{A_{0}(x)}{y-x}\right) d y,
$$

where $A_{i}(x)$ are $n \times n$-matrices, and apply to it a gauge change $(\alpha(x), B(x))$, $B(x) \in \mathrm{GL}_{n}$. We get

$$
\begin{aligned}
& B^{-1}(x) \cdot P(\alpha(x), \alpha(y)) \cdot B(y)=\left(\ldots+\frac{\tilde{A}_{-2}(x)}{(y-x)^{3}}+\frac{\tilde{A}_{-1}(x)}{(y-x)^{2}}+\frac{\tilde{A}_{0}(x)}{y-x}\right. \\
& +\left(\tilde{A}_{0}(x) \cdot F_{0}^{1}\left(\alpha^{\prime-1} \alpha^{\prime \prime}, B^{-1} B^{\prime}\right)\right)+\tilde{A}_{-1} \cdot F_{-1}^{1}\left(\alpha^{\prime-1} \alpha^{\prime \prime}, B^{-1} B^{\prime}\right)+\ldots \\
& \left.\ldots+\left(\tilde{A}_{0}(x) F_{0}^{2}\left(\alpha^{\prime-1} \alpha^{\prime \prime}, B^{-1} B^{\prime}\right)+\ldots\right)(y-x)+\ldots\right) d y,
\end{aligned}
$$

where $F_{j}^{i}$ are certain universal isobar differential polynomials. Then, if we have connections $\nabla_{\mathscr{T}}$ (where $\left.\mathscr{T}:=\omega^{-1}\right), \nabla_{E}$ with potentials $\Phi_{\mathscr{T}} d x, \Phi_{E} d x$, then the section

$$
S_{V \mathscr{T}, \nabla_{E}}: \mathscr{P}_{E} / \mathscr{P}_{E, 0}=\mathscr{D}_{E} \rightarrow \mathscr{P}_{E}
$$

is given in coordinates by the formula

$$
\begin{aligned}
s\left(\ldots+\frac{A_{-1}(x)}{(y-x)^{2}}+\frac{A_{0}(x)}{y-x}\right) d y & \\
= & \left(\ldots+\frac{A_{-1}(x)}{(y-x)^{2}}+\frac{A_{0}(x)}{y-x}+\left(A_{0}(x) \cdot F_{0}^{1}\left(\Phi_{\mathscr{T}}, \Phi_{E}\right)\right.\right. \\
& \left.\left.\quad+\mathrm{A}_{-1}(\mathrm{x}) \cdot \mathrm{F}_{1}^{1}\left(\Phi_{\mathscr{T}}, \Phi_{E}\right)+\ldots\right)+\ldots\right) d y .
\end{aligned}
$$


For example, we have [for the scalar matrix $a_{-1}(x)$ ],

$$
\begin{aligned}
& \left(\frac{a_{-1}(x)}{(y-x)^{2}}+\frac{A_{0}(x)}{y-x}\right) \mathrm{dy} \stackrel{(\alpha, B)}{\longrightarrow} B^{-1}(x)\left(\frac{a_{-1}(\alpha(x))}{(\alpha(y)-\alpha(x))^{2}}\right. \\
& \left.\quad+\frac{A_{0}(\alpha(x))}{\alpha(y)-\alpha(x)}\right) d \alpha(y) B(y)=\left(\frac{\tilde{a}_{-1}(x)}{(y-x)^{2}}+\frac{\tilde{A}_{0}(x)}{y-x}\right. \\
& \quad+\left(\tilde{A}_{0}\left(\frac{1}{2} \alpha^{\prime \prime} / \alpha^{\prime}+B^{-1} B^{\prime}\right)+\tilde{a}_{-1}\left(\frac{1}{6}\left(\alpha^{\prime \prime} / \alpha^{\prime}\right)^{\prime}-\frac{1}{12}\left(\alpha^{\prime \prime} / \alpha^{\prime}\right)^{2}\right.\right. \\
& \left.\left.-\frac{1}{2}\left(\alpha^{\prime \prime} / \alpha^{\prime}\right) \cdot B^{-1} B^{\prime}+\frac{1}{2}\left(B^{-1} B^{\prime}\right)^{\prime}-\frac{1}{2}\left(B^{-1} B^{\prime}\right)^{2}\right)+\ldots\right) d y,
\end{aligned}
$$

where

$$
\begin{aligned}
\tilde{a}_{-1}= & \alpha^{\prime-1} a_{-1}(\alpha(x)) ; \tilde{A}_{0}=B^{-1}(x) A_{0}(\alpha(x)) B(x) \\
& +\alpha^{\prime-1}(x) a_{-1}(\alpha(x)) B^{-1}(x) B^{\prime}(x) .
\end{aligned}
$$

So, for connections $\Phi_{E}, \Phi_{\mathscr{T}}$, the section

$$
S_{\nabla_{\mathscr{T}}, \nabla_{E}}: \mathscr{A}_{E}^{-1} \rightarrow^{\mathrm{tr}} \mathscr{A}_{E}^{-1}
$$

is given by the formula

$$
\begin{aligned}
\left(a_{-1}, A_{0}\right) \mapsto & \left(a_{-1}, A_{0}, \operatorname{tr}\left[A_{0}\left(\frac{1}{2} \Phi_{\mathscr{T}}+\Phi_{E}\right)+a_{-1}\right.\right. \\
& \left.\left.\times\left(\frac{1}{6} \Phi_{\mathscr{T}}^{\prime}-\frac{1}{12} \Phi_{\mathscr{T}}^{2}-\frac{1}{2} \Phi_{\mathscr{T}} \Phi_{E}+\frac{1}{2} \Phi_{E}^{\prime}-\frac{1}{2} \Phi_{E}^{2}\right)\right]\right) .
\end{aligned}
$$

Remark. The described above $F_{i}^{j}$ are the unique invariant differential polynomials giving the desired section.

\section{4. $\mathrm{C}^{\infty}$-Connection on $\mathscr{C}^{\text {tr }} \mathscr{A}_{E}$}

Return to the relative situation $\pi: X \rightarrow S$. Let $z$ denote a local coordinate along the fiber of $\pi, \bar{\partial}_{z}-a$ fiberwise $\bar{\partial}$-operator (respectivbly, $\bar{\partial}$-- the full $\bar{\partial}$-operator on $X$ ). Let $\nabla_{T}, \nabla_{E}$ be $\mathrm{C}^{\infty}$-connections on $\mathscr{T}_{X / S}, E$ respectively, with $(1,0)$-components (locally) $\nabla_{E, \partial_{z}}^{1,0}=\partial_{z}+\Phi_{E} d z, \nabla_{T, \partial_{z}}^{1,0}=\partial_{z}+\Phi_{T} d z, \Phi_{E} \in \mathrm{Mat}_{n} . \nabla_{T}$ induces connection $\nabla_{\omega}$ on $\omega=\omega_{X / S}$ with $\nabla_{\omega, \partial_{z}}^{1,0}=\partial_{z}-\Phi_{T} d z$. Let $\mathrm{C}\left(\nabla_{E}\right) \in \Omega_{X}^{1,1} \otimes \mathrm{Mat}_{n}, \mathrm{C}\left(\nabla_{T}\right)=-\mathrm{C}\left(\nabla_{\omega}\right) \in \Omega_{X}^{1,1}$ denote the $(1,1)$-components of the curvature and $\overline{\mathrm{C}}\left(\nabla_{E}\right)=\bar{\partial} \Phi_{E}, \overline{\mathrm{C}}\left(\nabla_{T}\right)=\bar{\partial} \Phi_{T}$ [respectively, $\left.\widetilde{C}\left(\nabla_{E}\right)=\bar{\partial}_{z} \Phi_{E}, \widetilde{C}\left(\nabla_{T}\right)=\bar{\partial}_{z} \Phi_{T}\right)$ - the image of $\mathrm{C}$ under the projection $\Omega_{X}^{1,1} \rightarrow \omega \otimes \Omega_{X}^{0,1}$ (respectively, $\Omega_{X}^{1,1} \rightarrow \Omega_{X / S}^{1,1}$ ). Finally, put $\mathrm{c}_{1}=\operatorname{trC}$, with the same meaning of ${ }^{-}$and ${ }^{\sim} . \mathrm{c}_{1}$ is the (1,1)-component of the first Chern class.

Put for brevity $\hat{\mathscr{A}}_{E / S}={ }^{\text {tr }} \mathscr{A}_{E / S}^{-1}$, and let $\mathscr{C} \hat{\mathscr{A}}_{E / S}$ be its push-forward by a map $\omega \rightarrow \Omega_{X \mid S}^{1}, 0$. The construction of the preceding subsection gives the section,

$$
s=s_{\nabla_{E}, \nabla_{T}}: \mathscr{A}_{E / S} \rightarrow \mathscr{C} \hat{\mathscr{A}}_{E / S} .
$$

\subsubsection{Lemma.}

$$
\begin{aligned}
\bar{\partial} s\left(a_{-1}, A_{0}\right)= & \operatorname{Tr}\left[\left(A_{0}-a_{-1} \Phi_{E}\right)\left(\frac{1}{2} \bar{C}\left(\nabla_{T}\right)+\bar{C}\left(\nabla_{E}\right)\right)\right. \\
& \left.+\nabla_{\omega, a_{-1} \partial_{z}}\left(n / 6 \bar{c}_{1}\left(\nabla_{T}\right)-\frac{1}{2} \bar{c}_{1}\left(\nabla_{E}\right)\right)\right] .
\end{aligned}
$$


Proof. From (5.3.2.1) we have

$$
\begin{aligned}
\bar{\partial} s\left(a_{-1}, A_{0}\right)= & \operatorname{Tr}\left[a_{0}\left(\bar{\partial} \Phi_{E}+\frac{1}{2} \bar{\partial} \Phi_{T}\right)+a_{-1}\left(\frac{1}{2} \partial_{z} \bar{\partial} \Phi_{E}-\Phi_{E} \bar{\partial} \Phi_{E}\right.\right. \\
& \left.\left.-\frac{1}{2} \bar{\partial} \Phi_{E} \Phi_{T}-\frac{1}{2} \Phi_{E} \bar{\partial} \Phi_{T}+\frac{1}{6} \partial_{z} \bar{\partial} \Phi_{T}-\frac{1}{6} \Phi_{T} \bar{\partial} \Phi_{T}\right)\right] \\
= & \operatorname{Tr}\left[\left(A_{0}-a_{-1} \Phi_{E}\right)\left(\frac{1}{2} \bar{\partial} \Phi_{T}+\bar{\partial} \Phi_{E}\right)+a_{-1} \cdot\left(\partial_{z}-\Phi_{T}\right)\left(\frac{1}{6} \bar{\partial} \Phi_{T}+\frac{1}{2} \bar{\partial} \Phi_{E}\right)\right] .
\end{aligned}
$$

5.4.2. Now we can construct the desired $\mathrm{C}^{\infty}$-connection on $\mathscr{C}^{\text {tr }} \mathscr{A}_{E}$. The last algebra is the complex

$$
\begin{aligned}
& \Omega_{X / S}^{00} \longrightarrow \Omega_{X / S}^{0,1} \oplus \mathscr{C} \hat{\mathscr{A}}_{E / S} \stackrel{\left(\begin{array}{ll}
\hat{o}_{z} & 0 \\
\hat{\partial}_{z} & \delta
\end{array}\right)}{\longrightarrow} \Omega_{X / S}^{1,1} \oplus \mathscr{A}_{E, \pi} . \\
& \begin{array}{lll}
-2 & -1 & 0
\end{array}
\end{aligned}
$$

We must construct the section $s_{\nabla}$ of the evident projection of this complex to

We put

$$
\mathscr{A}_{E}=\left(\mathscr{A}_{E / S} \longrightarrow \mathscr{A}_{E, \pi}\right) \text {. }
$$

$$
\begin{aligned}
s_{\nabla}^{-1}: \mathscr{A}_{E / S} \rightarrow \Omega_{X / S}^{0,1} \oplus \mathscr{C}_{E / S}, \\
s_{\nabla}^{-1}(a)=\left(0, s_{\nabla_{E}, \nabla_{T}}(a)\right) ; \\
s_{\nabla}^{0}: \mathscr{A}_{E, \pi} \rightarrow \Omega_{X / S}^{1,1} \oplus \mathscr{A}_{E, \pi}, \\
s_{\nabla}^{0}(a)=\left(\nabla_{\omega, \varepsilon(a)}\left(\frac{1}{6} \tilde{\mathrm{c}}_{1}\left(\nabla_{\omega}\right)-\frac{1}{2} \tilde{\mathrm{c}}_{1}\left(\nabla_{E}\right)\right)\right. \\
\quad+\operatorname{Tr}\left[\pi_{\nabla_{E}}(a) \cdot\left(\frac{1}{2} \tilde{\mathrm{C}}\left(\nabla_{\omega}\right)-\tilde{\mathrm{C}}\left(\nabla_{E}\right), \mathrm{id}\right)\right],
\end{aligned}
$$

where $\varepsilon: \mathscr{A}_{E, \pi} \rightarrow \mathscr{T}_{\pi}$ is the projection, $\pi_{\nabla_{E}}: \mathscr{A}_{E} \rightarrow \operatorname{End} E \otimes \mathscr{C}_{X}, \pi_{\nabla_{E}}(a)=a-\nabla_{E}(\varepsilon(a))$.

From Lemma 5.4.1 it follows directly that the so-constructed map $s_{\nabla}: \mathscr{A}_{E} \rightarrow \mathscr{C}^{\text {tr }} \mathscr{A}_{E}$ commutes with differentials in $\mathscr{A}_{E}, \mathscr{C}^{\text {tr }} \mathscr{A}_{E}$, i.e. defines the $\mathrm{C}^{\infty}$ connection on $\mathscr{C}^{\text {tr }} \mathscr{A}_{E}$.

\section{5 .}

Proposition. Let $\mathrm{c}_{\nabla}$ be the $(1,1)$-component of the induced $\mathrm{C}^{\infty}$-connection $\nabla$ on $\pi\left(\mathscr{C}^{\operatorname{tr}} \mathscr{A}_{E / S}\right)=\mathscr{C} \mathscr{A}_{\operatorname{det} R \pi_{*} E}$. Then $\mathrm{c}_{\nabla}$ is expressed by the Riemann-Roch-Grothendieck formula,

$$
\mathrm{c}_{\nabla}=\int_{\pi}\left(\frac{n}{12} \mathrm{c}_{1}\left(\nabla_{T}\right)^{2}+\frac{1}{2} \mathrm{c}_{1}\left(\nabla_{T}\right) \mathrm{c}_{1}\left(\nabla_{E}\right)+\mathrm{ch}_{2}\left(\nabla_{E}\right)\right),
$$

where $\operatorname{ch}_{2}\left(\nabla_{E}\right)=\frac{1}{2} \operatorname{tr} \mathrm{C}\left(\nabla_{E}\right)^{2}$.

Proof. To compute the curvature of $\nabla$, we shall work with $\bar{\partial}_{z}$-resolutions of $\mathscr{A}_{E},{ }^{\text {tr }} \mathscr{A}_{E}$. Let $\mathscr{C} \mathscr{A}_{E, \pi}$ denote the push-forward of $\mathscr{A}_{E, \pi}$ by the map $\mathscr{A}_{E / S} \rightarrow \mathscr{A}_{E / S} \otimes \mathscr{C}_{X}$. Let $v \in \mathscr{T}_{S}$, and $\tilde{v} \in \mathscr{C} \mathscr{A}_{E, \pi}$ be any of its non-holomorphic lifting. The construction of Sect. (5.4.2) extends in the evident way to $\bar{\partial}_{z}$-resolutions of $\mathscr{A}_{E},{ }^{\text {tr }} \mathscr{A}_{E}$, and from Stokes formula it follows that

$$
\begin{aligned}
v\lrcorner \mathrm{c}_{\nabla}= & \int_{\pi}\left\{\bar{\partial}\left(s \bar{\partial} \tilde{v}+\nabla_{\omega, \varepsilon \tilde{v}}\left(\frac{n}{6} \overline{\mathrm{c}}_{1}\left(\nabla_{T}\right)-\frac{1}{2} \overline{\mathrm{c}}_{1}\left(\nabla_{E}\right)\right)\right\}\right. \\
& +\operatorname{Tr}\left[\pi_{\nabla_{E}}(\tilde{v}) \cdot\left(-\frac{1}{2} \overline{\mathrm{c}}\left(\nabla_{T}\right)-\overline{\mathrm{c}}\left(\nabla_{E}\right)\right)\right] .
\end{aligned}
$$


On the other hand we have

(1) $\bar{\partial} s \bar{\partial} \tilde{v}=[\bar{\partial}, s] \bar{\partial} \tilde{v}=\nabla_{\omega, \varepsilon(\bar{\partial} \tilde{v})}\left(\frac{n}{6} \overline{\mathrm{c}}_{1}\left(\nabla_{T}\right)+\frac{1}{2} \overline{\mathrm{c}}_{1}\left(\nabla_{E}\right)\right)$

$$
+\operatorname{Tr}\left[\pi_{\nabla_{E}}(\tilde{v}) \cdot\left(-\bar{c}\left(\nabla_{E}\right)-\frac{1}{2} \bar{c}\left(\nabla_{T}\right)\right)\right] \quad[\text { from (3.5.4.1)]; }
$$

(2) $\left.\bar{\partial} \nabla_{\omega, \varepsilon \tilde{v}}(\ldots)=-\varepsilon \tilde{v}\right\lrcorner \mathrm{c}_{1}\left(\nabla_{T}\right)+\nabla_{\omega, \varepsilon(\tilde{\partial} \tilde{v})}(\ldots)$;

(3) $\bar{\partial}\left(\pi_{\nabla_{E}}(\tilde{v})\right)=\bar{\partial}\left(\tilde{v}-\nabla_{E}(\varepsilon \tilde{v})=\partial \tilde{v}-\nabla_{E}(\varepsilon(\bar{\partial} \tilde{v})-\varepsilon \tilde{v}\lrcorner \overline{\mathfrak{c}}\left(\nabla_{E}\right)\right.$.

Hence

$$
\begin{aligned}
v\lrcorner \mathrm{c}_{\nabla}= & \int_{\pi}\left\{(\varepsilon \tilde{v}\lrcorner \mathrm{c}_{1}\left(\nabla_{T}\right)\right)\left(\frac{n}{6} \overline{\mathrm{c}}_{1}\left(\nabla_{T}\right)+\frac{1}{2} \overline{\mathrm{c}}_{1}\left(\nabla_{E}\right)\right) \\
& \left.\left.+\operatorname{Tr}\left[(\varepsilon \tilde{v}\lrcorner \overline{\mathrm{C}}\left(\nabla_{E}\right)\right)\left(\overline{\mathrm{C}}\left(\nabla_{E}\right)+\frac{1}{2} \overline{\mathrm{C}}\left(\nabla_{T}\right)\right)\right]\right\} \\
= & \left.\int_{\pi}\{\varepsilon \tilde{v}\lrcorner\left[\frac{n}{12} \mathrm{c}_{1}\left(\nabla_{T}\right)^{2}+\frac{1}{2} \mathrm{c}_{1}\left(\nabla_{T}\right) \mathrm{c}_{1}\left(\nabla_{E}\right)+\mathrm{ch}_{2}\left(\nabla_{E}\right)\right]\right\} \\
= & v\lrcorner \int_{\pi}\left(\frac{n}{12} \mathrm{c}_{1}\left(\nabla_{T}\right)^{2}+\frac{1}{2} \mathrm{c}_{1}\left(\nabla_{T}\right) \mathrm{c}_{1}\left(\nabla_{E}\right)+\operatorname{ch}_{2}\left(\nabla_{E}\right)\right) .
\end{aligned}
$$

5.6.

Remark. It seems very probable that when $\nabla_{E}, \nabla_{T}$ arise from hermitian metrics on $E, \mathscr{T}_{X / S}$, then $\pi(\nabla)$ is just the connection associated with the corresponding Quillen metric on $\lambda_{E},[18]$.

\section{Logarithmic Singularities}

\subsection{Atiyah Algebras with Logarithmic Singularities}

6.1.1. Let $X$ be a smooth variety, $D \subset X$ a smooth divisor. Let $\mathscr{T}_{X, D} \subset \mathscr{T}_{X}$ be the subalgebra of vector fields that preserve $D$. In the case $\operatorname{dim} X=1 \mathscr{T}_{X, D}=\mathscr{T}_{X}(-D)$. If $\mathscr{A}$ is an Atiyah algebra over $X$, put $\mathscr{A}(\log D):=\varepsilon^{-1}\left(\mathscr{T}_{X, D}\right) \subset \mathscr{A}$.

If $(\mathscr{A}, R),(\mathscr{B}, S)$ are two Atiyah algebras, then we call a morphism with logarithmic singularities at $D$, or simply a $\log D$-morphism, between $\mathscr{A}$ and $\mathscr{B}$ an $\mathcal{O}_{X}$-linear Lie algebra map $f: \mathscr{A}(\log D) \rightarrow \mathscr{B}(\log D)$ such that $f(R) \subset S,\left.f\right|_{R}: R \rightarrow S$ is an algebra map and the induced map $\mathscr{A} / R \rightarrow \mathscr{B} / S$ is an identity on $\mathscr{T}_{X . D}$. So if $D=\emptyset$, then $f$ is just the usual morphism of Atiyah algebras.

6.1.2. Example. If $\mathscr{A}=\mathscr{A}_{\mathbb{C}_{X}}, \mathscr{B}=\mathscr{A}_{E}$ (E is a vector bundle on $\left.X\right)$ then a $\log D$ morphism $\mathscr{A}(\log D) \rightarrow \mathscr{A}_{E}(\log D)$ is the same as an integrable connection on $E$ with logarithmic singularities at $D$.

6.1.3. Let $E$ be a vector bundle on $X$. Put

$$
\mathscr{D}_{E}(\log D):=\sum_{i \geqq 0} J^{i} \mathscr{D}_{E, i}=\left\{\partial \in \mathscr{D}_{E}: \partial_{E}\left(J^{i} E\right) \subset J^{i} E \text { for all } i \geqq 0\right\},
$$

where $J C O_{X}$ is an ideal defining $D$ (cf. 1.1.1). 
Clearly, $\mathscr{D}_{E, D} \subset \mathscr{D}_{E}$ is a subalgebra filtered by $\mathscr{D}_{E}(\log D)_{i}:=\mathscr{D}_{E}(\log D) \cap \mathscr{D}_{E, i}$ $=\sum_{k \leqq i} J^{k} \mathscr{D}_{E, k}$. From now on assume that $X$ is a curve. Then local sections of $\mathscr{D}_{E}(\log D)$ in a neighbourhood of $x \in D$ are just polynomials (with matrix coefficients) of $t \partial_{t}$, where $t$ is a local coordinate at $x$.

Similarly, put

$$
\begin{gathered}
\mathscr{P}_{E}(\log D)=\sum_{i} J^{i} \mathscr{P}_{E, i} \subset \mathscr{P}_{E} \\
\mathscr{P}_{E}(\log D)_{i}=\sum_{k \leqq i} J^{k} \mathscr{P}_{E, k} \mathscr{P}_{E}(\log D)_{i, j}=\mathscr{P}_{E}(\log D)_{i} / \mathscr{P}_{E}(\log D)_{j}
\end{gathered}
$$

(cf. 2.1.1.2). We have

$$
\begin{gathered}
\mathscr{P}_{E}(\log D) / \mathscr{P}_{E}(\log D)_{-1}=\mathscr{D}_{E}(\log D) \\
\mathscr{P}_{E}(\log D)_{i} / \mathscr{P}_{E}(\log D)_{i-1}=\mathscr{T}_{X}(-D)^{\otimes i} \otimes \text { End } E .
\end{gathered}
$$

Note that $\mathscr{T}_{X}(-D)^{\otimes-1}=\omega(\log D):=$ the sheaf of differentials with logarithmic singularities at $D$. Clearly, $\mathscr{P}_{E}(\log D)$ is $\mathscr{D}_{E}(\log D)$-bimodule, cf. 2.1.1.2.

The same definition works for arbitrary Atiyah algebra $\mathscr{A}$; thus we get the $\mathscr{D}_{\mathscr{A}}(\log D)$-bimodules, $\mathscr{P}_{\mathscr{A}}(\log D)$ etc. (cf. Appendix A2.1). One has of course

$$
\begin{aligned}
\mathscr{A}(\log D)= & \left\{\partial \in \mathscr{P}_{\mathscr{A}}(\log D)_{1,-1} \mid \operatorname{Symb} \partial:=\partial \bmod \mathscr{P}_{A}(\log D)_{0}\right. \\
& \left.\in \mathscr{T}_{X}(-D) \subset \mathscr{T}_{X}(-D) \otimes R\right\} .
\end{aligned}
$$

6.1.4. Functoriality of $\mathscr{P}(\log D)$ with Respect to $\log D$-Morphisms (cf. 5.3). Let $f: \mathscr{A}(\log D) \rightarrow \mathscr{B}(\log D)$ be a $\log D$-morphism. Then it canonically induces the map $\mathscr{P}_{f}: \mathscr{P}_{\mathscr{A}}(\log D) \rightarrow \mathscr{P}_{B}(\log D)$. In fact, since $\left.\mathscr{A}(\log D)\right|_{X-D}=\left.\mathscr{A}\right|_{X-D}, \mathscr{P}_{f}$ is defined on $X-D$ because $\mathscr{P}_{\mathscr{A}}$ is functorial with respect to $\mathscr{A}$ (cf. Appendix, A2.1). We can extend this map to the whole $X$ and have only to verify that $\mathscr{P}_{\mathscr{A}}(\log D)$ is mapped into $\mathscr{P}_{\mathscr{B}}(\log D)$.

To see this we shall write down the local formulas for $\left.\mathscr{P}_{f}\right|_{X-D}$. For simplicity we'll restrict ourselves to the case $\mathscr{A}=\mathscr{A}_{E}, \mathscr{B}=\mathscr{A}_{F}, \mathrm{rk} E=n, \mathrm{rk} F=m$. Let us follow the lines of 5.3.2. Consider the expression

$$
\sum_{i \leq i_{0}} A_{i}(x) d y(y-x)^{i-1}, \quad A_{i}(x) \in \mathrm{Mat}_{m}
$$

and perform with it a gauge transform $\left(B(x) \in \mathrm{GL}_{m}\right)$ :

$$
\begin{aligned}
& B^{-1}(x) \sum A_{i}(x) d y(y-x)^{i-1} B(y)=\sum_{i \leq i_{0}}\left(\sum_{i \leq j \leq i_{0}} B^{-1}(x) A_{j}(x) B(x)\right. \\
& \left.\quad \times P_{j-i}\left(B^{-1}(x) B^{\prime}(x),\left(B^{-1}(x) B^{\prime}(x)\right)^{\prime}, \ldots,\left(B^{-1}(x) B^{\prime}(x)\right)^{(j-i-1)}\right)\right) \\
& \quad \times d y(y-x)^{i-1},
\end{aligned}
$$

where

$$
P_{k}\left(B^{-1}(x) B^{\prime}(x),\left(B^{-1}(x) B^{\prime}(x)\right)^{\prime}, \ldots,\left(B^{-1}(x) B(x)\right)^{(k-1)}\right)=\frac{1}{k !} B^{-1}(x) B^{(k)}(x) .
$$


Now choose a local coordinate $x$ on $X$, hence $(x, y)$ on $X \times X$, and local trivialisations of $E$ and $F$. Suppose that $f: \mathscr{A}_{E} \rightarrow \mathscr{A}_{F}$ in this local coordinates has the form

$$
\begin{gathered}
f\left(a(x) \partial_{x}+A(x)\right)=a(x) \Phi(x)+f(A)(x)+a(x) \partial_{x}, \\
a(x) \in \mathcal{O}_{X}, A(x) \in \operatorname{End}\left(\mathcal{O}_{X}^{n}\right), \Phi(x), f(A)(x) \in \operatorname{End}\left(\mathcal{O}_{X}^{m}\right)
\end{gathered}
$$

Then, if

$$
p=\left(A_{i_{0}}(x), A_{i_{0}-1}(x), \ldots\right):=\sum_{i \leq i_{0}} A_{i}(x) d y(y-x)^{i-1} \in \mathscr{P}_{E,-i_{0}}
$$

we have

$$
\mathscr{P}_{f}(p)=\left(\ldots, \sum_{i \leq j \leq i_{0}} f\left(A_{j}\right)(x) P_{j-i}\left(\Phi(x), \Phi^{\prime}(x), \ldots, \Phi^{(j-i-1)}(x)\right), \ldots\right) \in \mathscr{P}_{F,-i_{0}} .
$$

If $f$ has logarithmic singularities at $D$ then one easily sees from (6.1.4.1) that the last formula defines a map from $\mathscr{P}_{E}(\log D)$ to $\mathscr{P}_{F}(\log D)$.

In the case of an arbitrary Atiyah algebra the same formula works.

6.1.5. Example. On $\mathscr{P}_{1,-2}$

$$
\mathscr{P}_{f}\left(A_{-1}, A_{0}, A_{1}\right)=\left(f\left(A_{-1}\right), f\left(A_{-1}\right) \Phi+A_{0}, \frac{1}{12} f\left(A_{-1}\right)\left(\Phi^{2}+\Phi^{\prime}\right)+f\left(A_{0}\right)+A_{-1}\right) .
$$

6.1.6. Residue of a $\log D$-Morphism. Let $(\mathscr{A}, R),(\mathscr{B}, S)$ be Atiyah algebras and $f: \mathscr{A}(\log D) \rightarrow \mathscr{B}(\log D)$ a $\log D$-morphism. Let $\mathscr{B}(D)$ denote the push-forward of $B$ by means of $S \hookrightarrow S(D)$, where $S(D)=S \otimes \mathcal{O}(D)$. Then $f$ extends canonically to $f: \mathscr{A}$ $\rightarrow \mathscr{B}(D)$. The composition $\mathscr{A} \stackrel{f}{\longrightarrow} \mathscr{B}(D) \rightarrow \mathscr{B}(D) / \mathscr{B}=S(D) / S$ equals zero on $\mathscr{A}(\log D)$ and defines the map $\mathscr{T}_{X} / \mathscr{T}_{X}(-D)=\mathscr{A} / \mathscr{A}(\log D) \rightarrow S(D) / S=S \otimes \mathscr{T}_{X} / \mathscr{T}_{X}(-D)$, hence an element (which we call residue of $f$ ) $\operatorname{res}(f)=\sum_{x \in D} \operatorname{res}(f)_{x} \in \underset{x \in D}{\oplus} S_{x}$. Clearly, $\operatorname{res}(f)=0$ iff $f$ is induced by a morphism of Atiyah algebras $\mathscr{A} \rightarrow \mathscr{B}$.

6.1.7. Example. For $\nabla: \mathscr{A}_{\mathcal{O}}(\log D) \rightarrow \mathscr{A}_{E}(\log D) \operatorname{res}(\nabla)_{x}$ is just the operator $\nabla\left(t \partial_{t}\right)_{x} \in$ End $E_{x}$, where $t$ is a local coordinate at $x \in D$.

\subsection{Functoriality of Determinant}

6.2.1. Let $\pi: X \rightarrow S$ be a family of smooth proper curves, $i_{D}: D \hookrightarrow X$ a divisor étale over $S$. As usual, put $\omega=\Omega_{X / S}^{1}$.

Clearly, all constructions of 6.1 extend to this relative situation. In particular, for every Atiyah algebra $\mathscr{A}$ on $X$ one defines the relative Atiyah algebra $\mathscr{A}_{/ S}(\log D)$, the bimodule $\mathscr{P}_{\mathscr{A} / S}(\log D)$ etc. Also put $\mathscr{T}_{\pi . S}:=\mathscr{T}_{\pi} \cap \mathscr{T}_{D}, \mathscr{A}_{\pi}(\log D):=\mathscr{A}_{\pi}$ $\cap \mathscr{A}(\log D)$. Clearly, these objects are functorial with respect to $\log D$-morphisms. 6.2.2. Let $(\mathscr{A}, R),(\mathscr{B}, T)$ be Atiyah algebras over $X$ and $f: \mathscr{A}(\log D) \rightarrow \mathscr{B}(\log D)$ a $\log D$-morphism. We may apply the construction 6.1.6 fiberwise and obtain the section $\operatorname{res}(f) \in T_{D}:=i_{D}^{*} T$.

Lemma. For every $a \in \mathscr{A}(\log D)$ one has $\left[\operatorname{res}(f), f(a)_{D}\right]=0$, where $f(a)_{D}$ $\left.\in \mathscr{B}\right|_{D}:=\ddot{i}_{D}(\mathscr{B})$ is the restriction of $f(a)$ to $D[\mathrm{cf} .1 .1 .5(\mathrm{v})]$. 
Proof. Choose local coordinates $(t, s)$ on $X$ such that $t$ (respectively $s$ ) is a coordinate along a fiber (respectively along $S$ ) and a local equation of $D$ is $t=0$, and local connections $\mathscr{A} \cong \mathscr{T}_{X} \oplus R, \mathscr{B} \cong \mathscr{T}_{X} \oplus T$. In these coordinates $f$ has the form $f\left(a \partial_{t}+b \partial_{s}+r\right)=a \partial_{t}+b \partial_{s}+a \Phi+b \Psi+f(r), \quad \Phi=\tilde{\Phi} / t, \quad \widetilde{\Phi}$ holomorphic. Put $\mathscr{A}_{t}:=\left[\partial_{t}, A\right], A_{s}:=\left[\partial_{s}, A\right]$ for $A \in T$ or $R$. Since $f$ is a Lie algebra morphism, $f\left(r_{t}\right)$ $-f(r)_{t}=[\Phi, f(r)],[\Phi, \Psi]=\Phi_{s}-\Psi_{t}$. Taking the residue at $t=0$, we get $[\operatorname{res} \Phi, f(r)]=0, \quad[\operatorname{res} \Phi, \Psi]=\operatorname{res} \Phi$, hence $\left[\operatorname{res} \Phi,\left.\left(a \partial_{t}+b \partial_{s}+a \Phi+b \Psi\right)\right|_{t=0}\right]=0$ $(a(0, s)=0)$.

6.2.3. Corollary. Suppose that $B$ has trace. Then all functions $\operatorname{tr} \operatorname{res}(f)^{i} \in \mathcal{C}_{D}, i \geqq 0$, are locally constant.

Proof. By definition of trace, 1.1.7 we have

$$
0=\operatorname{tr}\left[\operatorname{res}(f)^{i}, f(a)_{D}\right]=\varepsilon\left(f(a)_{D}\right)\left(\operatorname{tr} \operatorname{res}(f)^{i}\right) \text { for every } a \in \mathscr{A}(\log D) .
$$

Since local sections $\varepsilon\left(f(a)_{D}\right)$ generate the whole tangent bundle $\mathscr{T}_{D}, \operatorname{tr} \operatorname{res}(f)^{i}$ is locally constant.

6.2.4. Let us call a $(\pi, D)$-algebra $\mathscr{A}$ on $X$ a filtered dg-Lie superalgebra together with a morphism $\varepsilon_{\mathscr{A}}: \mathscr{A}^{\cdot} \rightarrow \mathscr{T}_{\pi, D}^{\cdot}:=\left(\mathscr{T}_{X / S} \cap \mathscr{T}_{\pi, D} \rightarrow \mathscr{T}_{\pi, D}\right)$ satisfying axioms 1.2.1 with $\mathscr{T}_{\pi}$ replaced by $\mathscr{T}_{\pi, D}$. One has a functor $\mathscr{A} \sim \rightarrow \mathscr{A}_{D}:(\pi$-algebras $) \rightarrow((\pi, D)$ algebras) (pull-back by $\mathscr{T}_{\pi, D} \rightarrow \mathscr{T}_{\pi}$ ) [cf. 2.3.2(ii)].

Let $\mathscr{A}^{\circ}$ be a $(\pi, D)$-algebra and $\mathscr{Q}$ an $\mathcal{O}$-Atiyah algebra on $D$. Define a $(\pi, D)$ algebra $\mathscr{A}(\mathscr{Q})^{\cdot}$ as follows. Put $\mathscr{A}(\mathscr{Q})^{i}=\mathscr{A}^{i}$ for $i \neq 0,-1, \mathscr{A}(\mathscr{Q})^{-1}=j_{*} \mathscr{A}^{-1}$, where $j: \omega \rightarrow \omega(\log D)$. Next, put $\mathscr{A}(\mathscr{Q})^{0}$ to be the fiber product of $\mathscr{A}^{0}$ and $\mathscr{Q}$ over $\mathscr{T}_{D}$. Let $d^{-2}: \mathscr{A}(\mathscr{Q})^{-2} \rightarrow \mathscr{A}(\mathscr{Q})^{-1}$ be induced by the de Rham differential. Finally, let $d^{-1}$ be the product of the map $\mathscr{A}(\mathscr{Q})^{-1} \rightarrow \mathscr{A}^{0}$ induced by $d_{\mathscr{A}}^{-1}$ and the sum of residues $\mathscr{A}(\mathscr{2})^{-1} \rightarrow \mathscr{A}(\mathscr{2})^{-1} / \mathscr{A}^{-1}=\omega(\log D) / \omega \rightarrow \mathcal{O}_{D} \hookrightarrow \mathscr{2}$. For a $\pi$-algebra $\mathscr{A}$, we'll denote $\mathscr{A}_{D}(\mathscr{Q})$ simply by $\mathscr{A}(\mathscr{Q})$.

6.2.5. Lemma [cf. 2.3.2(ii)]. One has canonical isomorphism of $\mathcal{O}_{S}$-Atiyah algebras

$$
\pi(\mathscr{A} \cdot(\mathscr{Q}))=\pi(\mathscr{A})+\operatorname{tr}_{*} \pi \cdot \mathscr{Q},
$$

where $\pi^{*} \mathscr{2}$ is the direct image by étale map $\pi \mid D, 1.1 .5(\mathrm{v})$, and $\operatorname{tr}: \pi_{*} \mathcal{O}_{D} \rightarrow \mathcal{O}_{S}$.

Proof. Consider a dg-Lie algebra $\tilde{\mathscr{A}}^{\cdot}=\mathscr{A}_{D} \times_{\mathscr{T}_{D}} \mathscr{Q}$. One easily sees that $R^{0} \pi_{*}\left(\tilde{\mathscr{A}}^{*}\right)$ $=\pi\left(\mathscr{A}^{*}\right)+\pi \cdot \mathscr{2}$, and a natural inclusion $\tilde{A}_{\rightarrow} \mathscr{A}_{2}$ induces on $R^{0} \pi_{*}$ the map $\operatorname{tr}_{*}$.

6.2.6. Let $(\mathscr{A}, R),(\mathscr{B}, T)$ be Atiyah algebras with trace on $X, f: \mathscr{A}(\log D) \rightarrow \mathscr{B}(\log D)$ a $\log D$-morphism such that

for certain $n \in \mathbb{C}$.

$$
\left.\operatorname{tr}_{T} \cdot f\right|_{S}=n \cdot \operatorname{tr}_{R}
$$

6.2.6.2. Put

$$
\mu(f):=\operatorname{tr}_{T}\left(\frac{(\operatorname{res}(f)(\operatorname{res}(f)-1)}{2}\right)
$$

By 6.2.3 $\mu(f)$ is a locally constant function on $D$. Put $N=N_{X / D}=i_{D}^{*} \mathscr{T}_{X / S}$ to be the normal bundle. Let $\mu(f) \mathscr{A}_{N}$ denote an $\mathcal{O}_{D}$-Atiyah algebra on $D$ equal to $\left.\mu(f)\right|_{D_{l}} \mathscr{A}_{\left.N\right|_{D_{i}}}$ on a connected component $D_{i} \subset D$. 
6.2.6.3. For $r \in R_{D}:=i_{D}^{*} R$, put

$$
\operatorname{tr}_{f}(r):=\operatorname{tr}_{T}(f(r) \cdot \operatorname{res}(f)) \text {. }
$$

Lemma. $\operatorname{tr}_{f}$ is a trace on the Atiyah algebra $\left.\mathscr{A}\right|_{D}$.

Proof. For $\left.a \in \mathscr{A}\right|_{D}, \quad r \in R_{D}$, we have $\operatorname{tr}_{f}([a, r])=\operatorname{tr}_{T}([f a, f r] \cdot \operatorname{res} f)$ $=\operatorname{tr}_{T}([f a, f r \cdot \operatorname{res} f])=\varepsilon(a)\left(\operatorname{tr}_{T}(f r \cdot \operatorname{res} f)\right)=\varepsilon(a) \operatorname{tr}_{f}(r), \quad$ since by 6.2 .2 $[f a$, res $f]=0$.

Now we can formulate the main result of this section.

6.2.7. Theorem-Construction. $f$ induces a morphism of $\pi$-algebras

$$
n \cdot{ }^{\operatorname{tr}} \mathscr{A}_{D} \rightarrow{ }^{\operatorname{tr}} \mathscr{B}\left(\left.\operatorname{tr}_{f} \mathscr{A}\right|_{D}-\mu(f) \mathscr{A}_{N}\right) .
$$

Proof. Put $\mathscr{Q}=\left.\operatorname{tr}_{f} \mathscr{A}\right|_{D}-\mu(f) \mathscr{A}_{N}$. Let $\varphi^{-1}:{ }^{\operatorname{tr}} \cdot \mathscr{A}_{D}^{-1} \rightarrow{ }^{\operatorname{tr}} \mathscr{B}(\mathscr{Q})^{-1}$ be a map induced by $\mathscr{P}_{f / S}: \mathscr{P}_{\mathscr{A} / S}(\log D)_{1,-2} \rightarrow \mathscr{P}_{\mathscr{B} / S}(\log D)_{1,-2}$, cf. 6.1.4. By (6.2.6.1) this map equals the multiplication by $n$ on $\omega$. Put $\varphi^{-2}: \mathcal{O}_{X} \rightarrow \mathcal{O}_{X}$ to be the multiplication by $n$. Finally, $\mathscr{A}_{\pi, D}$ acts naturally on $N$ and also maps to $\mathscr{A}_{D}$. Hence we have maps $\mathscr{A}_{\pi, D} \rightarrow \mathscr{2}$ and $f: \mathscr{A}_{\pi, D} \rightarrow \mathscr{B}_{\pi, D}$. Let $\varphi^{0}: \mathscr{A}_{\pi, D} \rightarrow \mathscr{B}(\mathscr{Q})^{0}$ be their fiber product. Using the local formula 6.1 .5 one easily sees that $\varphi^{\circ}$ commutes with a differential and thus defines the desired map.

6.2.8. Corollary. One has canonical isomorphism of $\mathcal{O}_{\mathrm{S}}$-Atiyah algebras

$$
\pi\left({ }^{\operatorname{tr}} \mathscr{B}\right) \cong n \cdot \pi\left({ }^{\operatorname{tr}} \mathscr{A}\right)+\operatorname{tr}_{*} \pi_{*}\left(\mu(f) \mathscr{A}_{N}-\left.\operatorname{tr}_{f} \mathscr{A}\right|_{D}\right) .
$$

This follows from 6.2.4.1 and 6.2.5.

6.2.9. Corollary. Let $E$ be a bundle of rank $n$ on $X$ with integrable connection $\nabla$ with logarithmic singularities along $D$, and $L$ an arbitrary line bundle on $X$. Then

$$
\mathscr{A}_{\lambda(E \otimes L)}=n \cdot \mathscr{A}_{\lambda(L)}+\operatorname{tr}_{*} \pi_{*}\left(\mu(\nabla) \mathscr{A}_{N}-v(\nabla) \mathscr{A}_{\left.\mathrm{det} E\right|_{D}}\right),
$$

where $v(\nabla)=\operatorname{tr} \operatorname{res}(\nabla)$.

Proof. Apply 6.2 .7 to a $\log D$-morphism $\mathscr{A}_{L}(\log D) \rightarrow \mathscr{A}_{E \otimes L}(\log D)$ induced by $\nabla$.

6.2.10. Remark. This formula in some respect resembles the Lefschetz formula.

\subsection{Compatibility with Mumford Isomorphism}

6.3.1. Consider the following situation

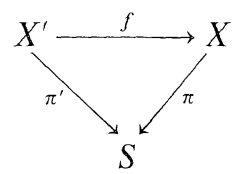

where $\pi, \pi^{\prime}$ are families of smooth proper curves, $f$ a covering of degree $n$ ramified at the divisor $i_{D}: D \hookrightarrow X$ étale over $S$. Put $D^{\prime}=f^{-1} D, \omega=\Omega_{X / S}^{1}, \omega^{j}=\omega^{\otimes j}, \omega^{\prime}=\Omega_{X^{\prime} / S}^{1}$, $N_{D}:=N_{X / D}, N_{D^{\prime}}:=N_{X^{\prime} / D^{\prime}}$ 
For line bundles $\mathscr{L}, \mathscr{L}^{\prime}$ over $S$ the notation $\mathscr{L} \simeq \mathscr{L}^{\prime}$ will mean an isomorphism between Atiyah algebras $\mathscr{A}_{\mathscr{L}} \cong \mathscr{A}_{\mathscr{L}^{\prime}}$. More generally, for $c \in \mathbb{C} \mathscr{L}^{\otimes c}$ in such a formula will mean $c \mathscr{A}$ in a corresponding formula for Atiyah algebras, cf. 1.1.6.

If $x \in D$ with local coordinate $t$ along $x, x^{\prime} \in f^{-1} x$ with ramification index $e$, then the local coordinate at $x^{\prime}$ is $t^{1 / e}$. On the sheaf $f_{*} \mathcal{O}_{X^{\prime}}$ arises a connection $\nabla$ with logarithmic singularities along $D$ induced by the trivial connection on $\mathcal{O}_{X^{\prime}}$. The spectrum of the operator $\nabla\left(t \partial_{t}\right)$ at $x$ is equal to $\left\{\frac{a}{e}, a=1, \ldots, e-1\right\}$. Thus,

$$
\mu(\nabla)(x)=\sum_{x^{\prime} \in f^{\prime}-1(x)} \frac{1-e_{x^{\prime}}^{2}}{12 e_{x^{\prime}}},
$$

since $\frac{1}{2} \sum_{a=1}^{e-1} \frac{a}{e}\left(\frac{a}{e}-1\right)=\frac{1-e^{2}}{12 e}$, cf. 6.1.7.

Hence we have from 6.2.9,

\subsubsection{Lemma.}

$$
\lambda_{f_{*} \mathbb{C}_{X^{\prime}}} \simeq \lambda_{\mathcal{O}_{X}^{n}} \cdot \operatorname{det} \pi_{*} N_{D}^{\Sigma \frac{1-e^{2}}{12 e}}
$$

6.3.3. Corollary. If $F$ is a line bundle on $X$ such that $\left.F\right|_{D} \cong \mathcal{O}_{D}$, then

$$
\lambda_{f^{*} F}=\lambda_{f_{*} \mathcal{O}_{X} \otimes F} \simeq \lambda_{F}^{\otimes n} \otimes \operatorname{det} \pi_{*} N_{D}^{\Sigma \frac{1-e^{2}}{12 e}} .
$$

Proof. Again use 6.2.9.

6.3.4. Lemma. $\lambda_{\omega^{\prime} J} \simeq \lambda_{\omega^{J}}^{n} \operatorname{det} \pi_{*} N_{D}^{c_{J} \Sigma \frac{1-e^{2}}{12 e}}, c_{j}=6 j^{2}-6 j+1$.

Proof. Since $\omega^{\prime}\left(\log D^{\prime}\right)=f^{*} \omega(\log D)$, we have by a projection formula

$$
\begin{aligned}
R \pi_{*}^{\prime} \omega^{\prime}\left(\log D^{\prime}\right)^{j} & =R \pi_{*} f_{*}\left(\omega^{\prime}\left(\log D^{\prime}\right)\right)^{j}=R \pi_{*} f_{*} f^{*}(\omega(\log D))^{j} \\
& =R \pi_{*}\left((\omega(\log D))^{j} \otimes f_{*} \mathcal{O}_{X^{\prime}}\right),
\end{aligned}
$$

whence from $6.3 .3\left[\right.$ since $\left.\left.\omega(\log D)\right|_{D}=\mathcal{O}_{D}\right]$,

(1) $\lambda_{\left(\omega^{\prime}\left(\log D^{\prime}\right)\right)^{\jmath}}=\lambda_{(\omega(\log D))^{\jmath} \otimes f_{*} \mathscr{C}_{X^{\prime}}} \simeq \lambda_{(\omega(\log D))^{j}}^{n} \otimes \operatorname{det} \pi_{*} N_{D}^{\Sigma \frac{1-e^{2}}{12 e}}$.

On the other hand we have the exact sequence

$$
0 \rightarrow \omega^{j} \rightarrow(\omega(\log D))^{j} \rightarrow \mathcal{O}_{X} / \mathcal{O}_{X}(-j D) \rightarrow 0
$$

and $\operatorname{det} \mathcal{O}_{X} / \mathcal{O}_{X}(-j D)=N_{D}^{-\frac{j(j-1)}{2}}$, whence

$$
\lambda_{\omega^{j}}=\lambda_{(\omega(\log D))^{j}} \cdot \operatorname{det} \pi_{*} N_{D^{\prime}}^{-\frac{j(j-1)}{2}},
$$

and an analogous formula for $\lambda_{\omega^{\prime}}$. Besides, we have $n=\sum e$ and

$$
\operatorname{det} \pi_{*} N_{D^{\prime}}=\operatorname{det} \pi_{*} N_{D}^{\Sigma \frac{1}{e}}
$$

Substituting this in (1), we obtain the desired equality. 
6.3.5. Proposition. Diagram

$$
\begin{gathered}
\lambda_{\omega^{\prime} j} \simeq \lambda_{\omega^{\prime}}^{n} \cdot \operatorname{det} \pi_{*} N_{D}^{c_{j} \cdot \sum \frac{1-e^{2}}{12 e}} \\
R \quad R \\
\lambda_{\omega^{\prime}}^{c_{1}} \simeq \lambda_{\omega^{\prime}}^{n c_{j}} \cdot \operatorname{det} \pi_{*} N_{D}^{c_{j} \cdot \sum \frac{1-e^{2}}{12 e}}
\end{gathered}
$$

where the vertical arrows are Mumford isomorphisms 3.1 .1 and horizontalisomorphisms 6.3.4, commutes.

Sketch of the Proof. Note that $\mathscr{T}_{\pi, D}$ acts on $(\omega(\log D))^{j}$ via the Lie derivative, so we can define a $(\pi, D)$-subalgebra $\mathscr{A}_{j[D]}^{\cdot} C^{\mathrm{tr}} \mathscr{A}_{(\omega(\log D))^{j}, D}$ that coincides with $\mathscr{T}_{\pi, D}$ in degree zero, cf. 3.1. One easily sees that the Mumford morphism (3.1.1) induces the morphism of $(\pi, D)$-algebras,

$$
c_{j} \cdot \mathscr{A}_{l[D]} \rightarrow \mathscr{A}_{j[D]}\left(\frac{j(j-1)}{2} \mathscr{A}_{N_{D}}\right),
$$

that after taking $\pi$ gives the isomorphism

$$
\lambda_{\omega(\log D)}^{c_{j}} \simeq \lambda_{(\omega(\log D))} \otimes N_{D}^{\underline{j(j-1)}} .
$$

Similarly, let $\mathscr{A}_{j[D]}^{\prime \prime}$ be a $(\pi, D)$-subalgebra of ${ }^{\operatorname{tr}} \mathscr{A}_{f_{*}\left(\omega^{\prime}\left(\log D^{\prime}\right)\right)^{J}, D}$ that coincides with $\mathscr{T}_{\pi, D}$ in degree zero and comes from the action of $\mathscr{T}_{\pi^{\prime}, D^{\prime}}$ on $\left(\omega^{\prime}\left(\log D^{\prime}\right)\right)^{j}$ and the map $\mathscr{T}_{\pi, D} \rightarrow f_{*} \mathscr{T}_{\pi^{\prime}, D^{\prime}}$. The map 6.2.7 induces the morphism

$$
n \cdot \mathscr{A}_{j[D]} \rightarrow \mathscr{A}_{j[D]}\left(\sum \frac{e^{2}-1}{12 e} \mathscr{A}_{N_{D}}\right)
$$

[cf. 6.3.4,(1)]. Now to prove our compatibility we have to verify the commutativity of the square

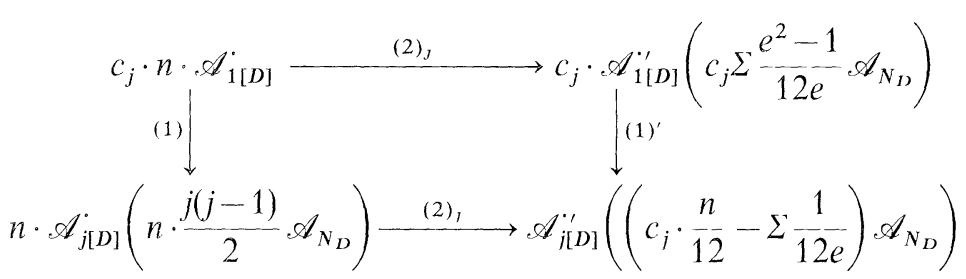

6.3.6. Remark. It is probable that 6.3 .4 will help to write down differential equations on Mumford forms for families of curves with given ramification over $\mathbb{P}^{1}$ generalizing 3.2.

6.3.7. Problem. The Mumford isomorphism is true not only for Atiyah algebras but also for corresponding sheaves: $\lambda_{\omega^{j}}=\lambda_{\omega}^{c_{j}}$, see 3.1.2. It would be very interesting to establish an analogous stronger version of 6.3.4:

$$
\lambda_{\omega^{\prime} j}^{12 I l e}=\lambda_{\omega^{J}}^{12 n I I e} \cdot \operatorname{det} \pi_{*} N^{\Sigma\left(1-e^{2}\right) \cdot \Pi e},
$$

cf. 2.7. Of course, the corresponding compatibility 6.3 .5 should hold. 


\section{Appendix. Local Riemann-Roch for $c_{1}$}

Our local Riemann-Roch is a canonical isomorphism between certain $\Omega$-extensions. We will start with the definition of terms in the Riemann-Roch formula.

\section{A1. O-Bilinear $\Omega$-Extensions}

These correspond to Chern classes in Riemann-Roch. Let $\mathscr{A}$ be an $R$-Atiyah algebra.

Definition. a) An $\Omega$-extension $\tilde{\mathscr{A}}$ of $(\mathscr{A}, R)$ is called $\mathcal{O}_{X}$-bilinear if []$_{-1,-1}: \tilde{\mathscr{A}}^{-1} \otimes \tilde{\mathscr{A}}^{-1} \rightarrow \tilde{\mathscr{A}}^{-2}=\mathcal{O}_{X}$ is $\mathcal{O}_{X}$-bilinear.

b) An orthogonal Atiyah algebra is a triple $(\mathscr{A}, R,()$,$) , where (\mathscr{A}, R)$ is an Atiyah algebra, and $():, R \otimes_{\mathcal{O}_{X}} R \rightarrow \mathcal{O}_{X}$ is a symmetric $\mathscr{O}_{X}$-bilinear $\mathscr{A}$-invariant pairing [i.e. for $a \in \mathscr{A}, r_{1}, r_{2} \in R$, we have $\left(r_{1}, r_{2}\right)=\left(r_{2}, r_{1}\right), \varepsilon(a)\left(r_{1}, r_{2}\right)=\left(\left[a, r_{1}\right], r_{2}\right)$ $\left.+\left(r_{1},\left[a, r_{2}\right]\right)\right)$.

An $\mathscr{O}_{X}$-bilinear extension $\mathscr{A}$ of $(\mathscr{A}, R)$ defines on $(\mathscr{A}, R)$ the orthogonal structure $(,)_{\mathscr{A}}$ : namely $(,)_{\mathscr{A}}$ is the pairing induced by $-\frac{1}{2}[,]_{-1,-1}$ on $\mathscr{A}_{-1}^{-1} / \mathscr{A}_{-2}^{-1}=R$. Clearly, both $\mathcal{O}_{X}$-bilinear extensions and orthogonal Atiyah algebras form a category, and $\left(\tilde{\mathscr{A}}^{\cdot}, \mathscr{A}, R\right) \sim \rightarrow\left(\mathscr{A}, R,(,)_{\mathscr{A}}\right)$ is a functor. We have $(,)_{\mathscr{A} \oplus \tilde{A}^{\prime}}=(,)_{\mathscr{A}} \oplus(,)_{\mathscr{A}^{\prime}},(,)_{\tilde{\mathscr{A}}+\tilde{I}^{\prime}}=(,)_{\mathscr{A}}+(,)_{\tilde{A}^{\prime}},(,)_{\lambda \mathscr{A}}=\lambda(,)_{\mathscr{A}}$.

A1.1. Claim. This functor is an equivalence of categories.

Proof. Let $\tilde{\mathscr{A}}$ be an $\mathcal{O}_{X}$-bilinear extension of $\mathscr{A}$. We will write $():,=(,)_{\tilde{\mathscr{A}}}$ for brevity. Define an admissible splitting of $\mathscr{A}$ to be an $\mathcal{O}_{X}$-isomorphism $s: \mathscr{T}_{X / S} \oplus R \oplus \Omega_{X / S}^{1} \stackrel{\sim}{\rightarrow} \mathscr{A}^{-1}$ that splits the filtration $\mathscr{A}^{-1}$ (hence $\left.s\right|_{\Omega^{1}}=\mathrm{id}_{\Omega^{1}}, \ldots$ ) and such that $\left.\mathscr{T}_{X / S} \perp\left(\mathscr{T}_{X / S} \oplus R\right), \Omega_{X / S}^{1} \perp \Omega_{X / S}^{1} \oplus R\right)$ with respect to the scalar product $\{\}:,=s^{*}[]_{-1,-1}$. Note that this $\{$,$\} does not depend on the choice of admissible$ $s$ : namely, it is given by formulas (here $\chi \in \mathscr{T}_{X / S}, r \in R, v \in \Omega_{X / S}^{1}$ ):

$$
\begin{gathered}
\left\{\chi, \chi^{\prime}\right\}=\left\{v, v^{\prime}\right\}=\{\chi, r\}=\{v, r\}=0 ; \\
\left\{r, r^{\prime}\right\}=-2\left(r, r^{\prime}\right),\{\chi, v\}=\chi v .
\end{gathered}
$$

An admissible splitting $s$ determines a relative connection $\nabla_{s}$ on $\mathscr{A}$ $\left(:=\mathscr{O}_{X}\right.$-linear section $\left.\nabla: \mathscr{T}_{X / S} \rightarrow \mathscr{A}_{\mid S}\right): \nabla_{S}=\left.S\right|_{\mathscr{T}_{X / S}} \bmod \Omega_{X / S}^{1}$.

A1.3. Lemma. $s \mapsto \nabla_{s}$ is 1-1 correspondence between admissible splittings and relative connections on $\mathscr{A}$.

Proof. Define the inverse map $\nabla \mapsto s_{\nabla}$ as follows. First, define $\left.s_{\nabla}\right|_{\mathscr{T}_{X / s}}$. Choose any $s: \mathscr{T}_{X / S} \rightarrow \tilde{\mathscr{A}}^{-1}$ such that $s \bmod \Omega^{1}=\nabla$, and put $s_{\mathcal{V}}(\chi)=s(\chi)$ $-\frac{1}{2}[s(\chi), s(\chi)]_{-1,-1} \cdot \chi^{-1}$. It is easy to see that the so-defined $s_{\nabla}: \mathscr{T}_{X / S} \rightarrow \tilde{\mathscr{A}}^{-1}$ is the only $\mathcal{O}_{X}$-linear map such that $s_{V} \bmod \Omega^{1}=\nabla$ and []$_{-1,-1}$ is zero on $s_{\nabla}\left(\mathscr{T}_{X / S}\right)$. Hence $S_{V}\left(\mathscr{T}_{X / S}\right) \oplus \Omega_{X / S}^{1}$ is a hyperbolic pair with respect to []$_{-1,-1}$. In particular []$_{-1,-1}$ is non-degenerate on $s_{\nabla}\left(\mathscr{T}_{X / S}\right) \oplus \Omega_{X / S}^{1}$. Let $s_{V}(R)$ be the orthogonal complement to this subspace. This defines the desired splitting.

For $(\chi, r, v) \in \mathscr{T}_{X / S} \oplus R \oplus \Omega_{X / S}^{1}$ and a relative connection $\nabla$, put $(\chi, r, v)_{\nabla}$ $=s_{\nabla}(\chi, r, v) \in \tilde{\mathscr{A}}^{-1}$. For $\omega \in \Omega_{X / S}^{1} \oplus R$ define ${ }^{\left(,{ }^{\prime}\right.} g_{\omega} \in \operatorname{Aut}_{\mathcal{O}_{X}}\left(\mathscr{T}_{X / S} \oplus R \oplus \Omega_{X / S}^{1}\right)$ by the 
formula,

$$
{ }^{(,)} g_{\omega}(\chi, r, v)=(\chi, \omega \chi+r,(\omega, \omega) \chi+2(\omega, r)+v) \text {. }
$$

Clearly ${ }^{(,)} g_{\omega_{1}+\omega_{2}}={ }^{\left({ }^{(,)}\right.} g_{\omega_{1}} \cdot{ }^{\left({ }^{(}\right)} g_{\omega_{2}}$, hence we get $\Omega_{X / S}^{1}$-action on $\mathscr{T}_{X / S} \oplus R \oplus \Omega_{X / S}^{1}$. It preserves the bilinear form $\{$,$\} .$

A1.5. Lemma. (i) $(\chi, r, v)_{\nabla+\omega}=\left({ }^{(,)} g_{\omega}(\chi, r, v)\right)_{\nabla}$.

(ii) For $a \in \mathscr{A}_{\pi}=\mathscr{A}^{0}$ one has (here $\operatorname{ad}_{a}(\nabla) \in \Omega_{X / S}^{1} \oplus R$ is defined by formula $\operatorname{ad}_{a}(\nabla) \chi=[a, \nabla(\chi)-\nabla([\varepsilon(a), \chi]):$

$$
\begin{aligned}
& {\left[a,(\chi, r, v)_{V}\right]_{0,-1}=\left([\varepsilon(a), \chi], \operatorname{ad}_{a}(\nabla) \chi+[a, r], 2\left(r, \operatorname{ad}_{a}(\nabla)\right)+\varepsilon(a) v\right)_{V}} \\
& {\left[\left(\chi_{1}, r_{1}, v_{1}\right),\left(\chi_{2}, r_{2}, v_{2}\right)\right]_{-1,-1}=\chi_{1} v_{2}+\chi_{2} v_{1}-2\left(r_{1}, r_{2}\right) \in \mathcal{O}_{X}=\tilde{\mathscr{A}}^{-2} .}
\end{aligned}
$$

The proof is plain calculation; note that the $\Omega_{X / S^{-}}^{1}$ component of $s_{\bar{V}}^{-1}(x)$, $x \in \tilde{\mathscr{A}}^{-1}$, is $\left[s_{V}(\chi), \chi\right]_{-1,-1} \cdot \chi^{-1}$, where $\chi$ is invertible section of $\mathscr{T}_{X / S}$.

Now we may prove A1.1. For an orthogonal Atiyah algebra $(\mathscr{A}, R,()$,$) define$ the $\mathcal{O}_{X}$-bilinear extension ${ }^{(,)} \tilde{\mathscr{A}}$ by formulas A1.5. More precisely, put ${ }^{(\cdot)} \tilde{\mathscr{A}}^{-1}$ to be $\mathscr{T}_{X / S} \oplus R \oplus \Omega_{X / S}^{1}$ twisted with the $\Omega_{X / S}^{1} \otimes R$-torsor of relative connections on $\mathscr{A}$ by the action (A 1.4). The elements of ${ }^{(,)} \mathscr{A}^{-1}$ are just $(\chi, r, v)_{\nabla}$ 's modulo equivalence relation A1.5(i). The bracket on ${ }^{(,)} \tilde{\mathscr{A}}$ is defined by formulas A1.5(ii). Clearly $(\mathscr{A},(),) \mapsto{ }^{(,)} \tilde{\mathscr{A}}$ is inverse to $\tilde{\mathscr{A}} \mapsto\left(\mathscr{A} ;(,)_{\mathscr{A}}\right)$.

\section{A2. Trace $\Omega$-Extension}

Here for any Atiyah algebra $\mathscr{A}$ with trace a canonical $\Omega$-extension of $\mathscr{A}$ will be defined. In the case $\mathscr{A}=\mathscr{A}_{E}$ and the usual trace this construction reduces to 2.1 .

Let $\mathscr{A}$ be an $R$-Atiyah algebra. Let us define the canonical $\mathscr{D}_{\mathscr{A} / S}$-bimodule $\mathscr{P}_{\mathscr{A}}$. A2.1. Put $R \mathcal{O}_{\hat{\Delta}}:=R \hat{\otimes} \mathcal{O}_{\Delta}=\lim R \otimes \mathcal{O}_{X \times{ }_{S} X} / \mathcal{O}_{X \times{ }_{S} X}(-i \Delta), \quad R \mathscr{P}_{i}:=R \hat{\otimes} \mathscr{P}_{i}$

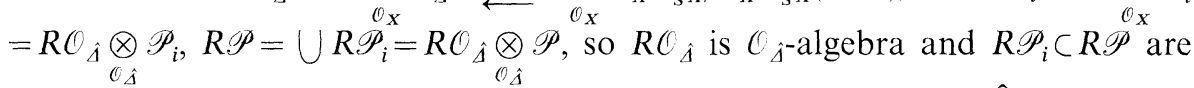
$R \mathcal{O}_{\hat{A}}$-bimodules. In the same way, we have the $\mathscr{O}_{\hat{A}}$-algebra $\mathcal{O}_{\hat{A}} R=\mathcal{O}_{\hat{A}} \hat{\otimes} R$ and $\mathcal{O}_{\hat{A}} R$ bimodules $\mathscr{P}_{i} R \subset \mathscr{P} R$.

Let $\nabla$ be a relative connection on $\mathscr{A}$. It defines the relative connection $\nabla_{R}$ on $R$, $\nabla_{R}(\chi)(r)=[\nabla(\chi), r], \chi \in \mathscr{T}_{X / S}, r \in R$. Hence we get

- the isomorphism of $\mathcal{O}_{\hat{\lambda}}$-algebras $I_{V}: R \mathcal{O}_{\hat{\lambda}} \simeq \mathcal{O}_{\hat{\lambda}} R, \quad I_{\nabla}(r f)=f \sum_{i \geqq 0} \frac{\left(x_{1}-x_{2}\right)^{i}}{i !}$

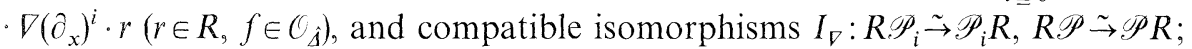

- the left $\mathscr{D}_{A / S}$-module structure on $R \mathcal{O}_{\hat{\Delta}}, R \mathscr{P}$, denoted by $(a, \alpha) \mapsto a \cdot{ }_{V} \alpha$, $a \in \mathscr{D}_{A / S}, \alpha \in R \mathscr{C}_{\hat{\lambda}}$ (or $R \mathscr{P}$ ), defined on generators $r \in R, \nabla(\chi) \in \mathscr{A}_{/ S}$ of $\mathscr{D}_{\mathscr{A} / S}$ by formula

$$
r \cdot{ }_{\nabla} \alpha=r \alpha, \quad \nabla(\chi) \cdot{ }_{\nabla} \alpha=\nabla_{R}^{(1)}(\chi)(\alpha),
$$

where $\nabla_{R}^{(1)}$ is differentiation by $\nabla_{R}$ along the first variable, $\nabla_{R}^{(1)}\left(\partial_{x}\right)\left(r \cdot f\left(x_{1}, x_{2}\right)\right)$ $=\nabla_{R}\left(\partial_{x}\right)(r) f\left(x_{1}, x_{2}\right)+r \partial_{x_{1}} f\left(x_{1}, x_{2}\right)$;

- in the same way, we have the right $\mathscr{D}_{A / S}$-module structure on $\mathscr{P} R(\beta, \alpha) \mapsto \beta \cdot{ }_{V} \alpha, \beta \in \mathscr{P} R, a \in \mathscr{D}_{A / S}$, such that $\beta \cdot{ }_{V} r=\beta r, \beta \cdot{ }_{V} \nabla(\chi)=\beta \cdot \nabla_{R}^{(2)}(\chi)[$ since $\mathscr{P}_{i}$, hence $\mathscr{P}_{i} R$ is right $\mathscr{D}_{X / S^{-}}$-module via $\left.\nabla_{R}^{(2)}\right]$.

Note that left and right $\mathscr{D}_{\mathscr{A} / \mathrm{S}^{-}}$-actions on $R \mathscr{P} \stackrel{I_{D}}{\cong} \mathscr{P} R$ commute, hence we have a $\mathscr{D}_{\mathscr{A} / \mathrm{S}}$-bimodule. Now we shall see that it actually does not depend on the choice of $\nabla$. 
For a pair $\nabla_{1}, \nabla_{2}$ of relative connections, $\nabla_{2}-\nabla_{1}=\omega \in R \otimes \Omega_{X / S}^{1}$, consider $f_{\nabla_{1} \nabla_{2}} \in R \mathcal{C}_{\hat{\Delta}}$ such that $f_{\nabla_{1} \nabla_{2}}(x, x)=1 \in R$,

$$
\nabla_{1 R}^{(1)}\left(f_{\nabla_{1} \nabla_{2}}\right)=f_{\nabla_{1} \nabla_{2}} \omega^{(1)} \quad\left(\text { here } \omega^{(1)}=p_{1}^{*} \omega \in R \mathcal{O}_{\hat{A}} \otimes p_{1}^{*} \Omega_{X / S}^{1}\right) .
$$

Such $f_{\nabla_{1} \nabla_{2}}$ exists and is unique; we have

$$
\nabla_{2 R}^{(1)}\left(f_{\nabla_{1} \nabla_{2}}\right)=\omega^{(1)} f_{\nabla_{1} \nabla_{2}}
$$

Same way, define $g_{\nabla_{2} \nabla_{1}} \in \mathcal{O}_{\Delta} R$ by properties $g_{\nabla_{2} \nabla_{1}}(x, x)=1$,

$$
\nabla_{1 R}^{(2)}\left(g_{V_{2} V_{1}}\right)=-\omega^{(2)} g_{\nabla_{2} \nabla_{1}}\left(\text { or } \nabla_{2 R}^{(2)}\left(g_{\nabla_{2} \nabla_{1}}\right)=-g_{\nabla_{2} \nabla_{1}} \omega^{(2)}\right) \text {. }
$$

A 2.1.1. Lemma. (i) $f_{\nabla_{1} \nabla_{2}} f_{\nabla_{2} \nabla_{3}}=f_{\nabla_{1} \nabla_{3}}, g_{\nabla_{3} \nabla_{2}} g_{\nabla_{2} \nabla_{1}}=g_{\nabla_{3} \nabla_{1}}$

(ii) $\left(a \cdot \nabla_{1} \alpha\right) f_{\nabla_{1} \nabla_{2}}=a_{\nabla_{2}} \cdot\left(\alpha f_{\nabla_{1} \nabla_{2}}\right), g_{\nabla_{2} \nabla_{1}}\left(\beta \cdot_{\nabla_{1}} a\right)=\left(g_{\nabla_{2} \nabla_{1}} \cdot \beta\right){ }_{\nabla_{2}} a$ $\left(a \in R \mathscr{P}, \beta \in \mathscr{P} R, a \in \mathscr{D}_{A / S}\right)$

(iii) $I_{\nabla_{2}}\left(\alpha f_{\nabla_{1} \nabla_{2}}\right)=g_{\nabla_{2} \nabla_{1}} I_{\nabla_{1}}(\alpha)$.

Define $\mathcal{O}_{\hat{A}}$-modules $\mathcal{O}_{\hat{A} \mathscr{A}}^{(1)}, \mathscr{P}_{\mathscr{A} \text { (r) }}^{(1)}$ (respectively, $\left.\mathscr{O}_{\hat{A} \mathscr{A}}^{(2)}, \mathscr{P}_{\mathscr{A}}^{(2)}\right)$ to be the limit of $R \mathcal{O}_{\hat{A}}$ 's, $R \mathscr{P}_{i}$ 's (respectively, $\mathcal{O}_{\hat{A}} R$ 's, $\mathscr{P}_{i} R$ 's), labelled by $\nabla$ 's, with respect to transition maps $\alpha_{\nabla_{1}} \mapsto \alpha_{\nabla_{2}}=\alpha_{\nabla_{1}} f_{\nabla_{1} \nabla_{2}}$ (respectively, $\beta_{\nabla_{1}} \mapsto \beta_{\nabla_{2}}=g_{\nabla_{2} \nabla_{1}} \beta_{\nabla_{1}}$ ); this is correct by (i) above. So for any relative connection $V$ we have isomorphisms $S_{V}^{(1)}: R \mathscr{G}_{\hat{1}} \stackrel{\sim}{\rightarrow} \mathscr{O}_{\hat{\lambda}}^{(1)}, R \mathscr{P}_{i} \stackrel{\sim}{\rightarrow} \mathscr{P}_{\mathscr{A}}^{(1)}$, respectively $S_{V}^{(2)}: \mathscr{O}_{\hat{A}} R \stackrel{\sim}{\rightarrow} \mathcal{O}_{\hat{A} \mathscr{A}}^{(2)}, \mathscr{P}_{i} R \stackrel{\sim}{\rightarrow} \mathscr{P}_{\mathscr{A} i}^{(2)}$, such that

$$
\alpha_{V_{1}}^{(1)}=\left(\alpha f_{V_{1} \nabla_{2}}\right)_{V_{2}}^{(1)}, \quad \beta_{V_{1}}^{(2)}=\left(g_{V_{2} \nabla_{1}} \beta\right)_{V_{2}}^{(2)},
$$

where $\alpha_{V}^{(1)}:=S_{\nabla}^{(1)}(\alpha), \beta_{\nabla}^{(2)}:=S_{V}^{(2)}(\beta)$.

According to (ii) above, $\mathcal{O}_{A \mathscr{A}}^{(1)}, \mathscr{P}_{\mathscr{A}}^{(1)}$ have natural left $\mathscr{D}_{A / S}$-action defined by formula $a \alpha_{\nabla}^{(1)}=\left(a{ }_{\nabla} \alpha\right)^{(1)}$; in the same way, $\mathscr{P}^{(2)}$ have right $\mathscr{D}_{\mathscr{A} / S}$-action $\beta_{\nabla}^{(2)} a$

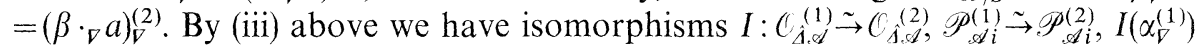
$=\left(I_{\nabla}(\alpha)\right)_{\nabla}^{(2)}$. These modules, identified via $I$, will be denoted simply $\mathscr{U}_{\hat{A} \mathscr{A}}, \mathscr{P}_{\mathscr{A} i}$. The above left and right $\mathscr{D}_{\mathscr{A} / S}$-actions define on $\mathscr{P}_{\mathscr{A}}$ the structure of $\mathscr{D}_{\mathscr{A} / S}$-bimodule. Clearly $\mathscr{P}_{\mathscr{A},-1} \subset \mathscr{P}_{\mathscr{A}}$ is $\mathscr{D}_{\mathscr{A} / S}$-biinvariant and we have canonical isomorphism $\mathscr{P}_{\mathscr{A}} / \mathscr{P}_{\mathscr{A},-1} \cong \mathscr{D}_{\mathscr{A} / S}$ of $\mathscr{D}_{\mathscr{A} / S}$-bimodules, $(r \otimes \partial)_{V}^{(1)} \mapsto r \nabla(\partial)\left(r \in R, \partial \in \mathscr{P} / \mathscr{P}_{-1}=\mathscr{D}_{X / S}\right.$, $\nabla(\partial) \in \mathscr{D}_{\mathscr{A} / S}$ ). These modules also carry a natural $\mathscr{A}_{\pi}$-action (since $\mathscr{A}_{\pi}$ acts by infinitesimal automorphisms on $\left(X, S, \pi, \mathscr{A}_{X / S}\right)$. Explicit formula: for $a \in \mathscr{A}_{\pi}, r \in R$, $f \in \mathscr{P}$ we have $\operatorname{Lie}_{a}\left((r f)_{V}^{(1)}\right)=\left([a, r] f+r \operatorname{Lie}_{\varepsilon(a)}(f)+r f \pi_{a}^{\nabla}\right)^{(1)}$, where $\pi_{a}^{V} \in R \mathcal{O}_{\Delta}$ is defined by properties $\pi_{a}^{\nabla}(x, x)=0$,

$$
\nabla_{R}^{(1)} \pi_{a}^{\nabla}=\operatorname{ad}_{a}(\nabla)\left(\text { i.e. } \pi_{a}^{\nabla}\left(x_{1}, x_{2}\right)=\int_{x_{1}}^{x_{2}} \operatorname{ad}_{a}(\nabla)\right) .
$$

A2.2. If $\mathscr{A}=\mathscr{A}_{E}$ then $\mathscr{O}_{\hat{A} \mathscr{A}} \cong E \otimes \mathcal{O}_{\hat{A}} \otimes E^{*}, \mathscr{P}_{\mathscr{A}} \cong E \otimes \mathscr{P} \otimes E^{*}=\mathscr{P}_{E}$ via the isomorphisms $\quad S_{V}^{(1)}:$ End $E \cdot \mathscr{O}_{\hat{A}}=\left(E \otimes E^{*}\right) \hat{\otimes} \mathcal{O}_{\hat{A}} \tilde{\rightarrow} E \otimes \mathcal{O}_{\hat{\lambda}} \otimes E^{*}, \quad$ End $E \cdot \mathscr{P}=\left(E \otimes E^{*}\right) \hat{\otimes} \mathscr{P}$ $\stackrel{\sim}{\rightarrow} \otimes \mathscr{P} \otimes E^{*}$ that come from isomorphism $E^{*} \hat{\otimes} \mathcal{O}_{\hat{A}} \cong \mathcal{O}_{\hat{A}} \hat{\otimes} E^{*}$ induced by $\nabla$. These isomorphisms are compatible with $\mathscr{D}_{\mathscr{A} / S^{-}}$and $\mathscr{A}_{\pi}$-action.

A2.3. Now assume that $\mathscr{A}$ has trace tr. Define the pairing \langle\rangle$_{\mathscr{A}, \mathrm{tr}}: \mathscr{P}_{\mathscr{A}} \otimes \mathscr{P}_{\mathscr{A}} \rightarrow \mathcal{C}_{X}$ by formula

$$
\left\langle\left(r_{1} \otimes \varphi_{1}\right)_{V}^{(1)},\left(\varphi_{2} \otimes r_{2}\right)_{V}^{(2)}\right\rangle_{\mathscr{A} \cdot \mathrm{tr}}:=\tilde{\operatorname{Res}} p_{2}^{*}\left(\operatorname{tr}\left(r_{1}, r_{2}\right)\right) \varphi_{1} \cdot{ }^{t} \varphi_{2}
$$

where $r_{i} \in R, \varphi_{i} \in \mathscr{P}$. 
Lemma. \langle\rangle$_{\mathscr{A}, \mathrm{tr}}$ is correctly defined symmetric $\mathscr{A}$-invariant pairing.

At this point we may repeat the construction 2.1.2 word-by-word to get the desired $\Omega$-extension ${ }^{\operatorname{tr}} \mathscr{A}$ of $\mathscr{A}$. By A $2.2^{\mathrm{tr}} \mathscr{A}_{E}$ so defined (for tr being equal to the standard trace on End $E$ ) coincides with the one from 2.1.2.

A2.4. Formulas. Define a (formal) $\mathscr{A}$-parametrix $\Pi_{\mathscr{A}}$ to be any section of $\mathscr{P}_{\mathscr{A}}$ that maps to $1 \in \mathscr{D}_{\mathscr{A} / S}$ by canonical projection $\mathscr{P}_{\mathscr{A}} \rightarrow \mathscr{D}_{\mathscr{A} / S}$; an $i$-jet of parametrix is a section of $\mathscr{P}_{\mathscr{A}} / \mathscr{P}_{\mathscr{A},-i}$ with the same property. A parametrix $\Pi_{\mathscr{A}}$ is the same as a section $s_{\Pi_{\mathscr{A}}}$ of canonical projection that commutes with left $\mathscr{D}_{\mathscr{A} / \mathrm{S}}$-action: $\Pi_{\mathscr{A}}=s_{\Pi_{\mathscr{A}}}(1), s_{\Pi_{\mathscr{A}}}(a)=a \Pi_{\mathscr{A}}$. In particular, a 2-jet ${ }^{(2)} \Pi_{\mathscr{A}}$ defines an $\mathcal{O}_{X}$-linear section $s_{I_{\mathscr{A}}}: \mathscr{A}_{/ S} \rightarrow^{\mathrm{tr}} \mathscr{A}^{-1}$.

Let $\Pi$ be an $\mathscr{A}_{\mathscr{C}_{X}}$-parametrix (we have $\Pi=\left(\frac{1}{x_{2}-x_{1}}+\Pi_{0}\left(x_{1}\right)\right.$ $\left.\left.+\left(x_{2}-x_{1}\right) \Pi_{1}\left(x_{1}\right)+\ldots\right) d x_{2}\right), x$ is a coordinate along the fiber), and let $\nabla$ be a relative connection on $\mathscr{A}$. We get the $\mathscr{A}$-parametrix $\Pi_{\nabla}:=(1 \otimes \Pi)_{V}^{(1)}$ (in case $\mathscr{A}=\mathscr{A}_{E} \Pi_{\nabla}$ is a product of $\Pi$ and $\nabla$-horizontal section of $E \otimes E^{*}$ that restricts to id on 4$)$, hence the isomorphism $\mathscr{T}_{X / S} \oplus R \oplus \Omega_{X / S}^{1} \tilde{\sim}^{\mathrm{tr}} \mathscr{A}^{-1}, \quad(\chi, r, v) \mapsto(\chi, r, v)_{\nabla, \Pi}$ $=s_{\Pi_{V}}(\nabla(\chi)+r)+v$. It depends on $\nabla$ and $\Pi$ as follows [here $f^{\prime}$ means $\hat{\partial}_{x} f$ for $f \in \mathcal{O}_{X}$, $r^{\prime}=\nabla_{R}\left(\partial_{x}\right)(r)$ for $\left.r \in R\right]$ :

$$
\begin{aligned}
& \left(\chi \partial_{x}, r, v d x\right)_{\nabla+\omega d x, \Pi} \\
& \quad=\left(\chi \partial_{x}, \omega \chi+r,\left(v+\chi \operatorname{tr}\left(\frac{1}{2}\left(\omega^{2}+\omega^{\prime}\right)-\omega \Pi_{0}\right)+\operatorname{tr}(r \omega)\right) d x\right) \\
& \left(\chi \partial_{x}, r, v d x\right)_{\nabla, I+\alpha_{0}\left(x_{1}\right) d x_{2}+\left(x_{2}-x_{1}\right) \alpha_{1}\left(x_{1}\right) d x_{2}} \\
& \quad=\left(\chi \partial_{x}, r,\left(v+\operatorname{tr}\left(\chi \alpha_{0}^{\prime}+r \alpha_{0}-\chi \alpha_{1}\right)\right) d x\right) .
\end{aligned}
$$

To give the formula for the bracket assume that $\Pi=\frac{d x_{2}}{x_{2}-x_{1}}$. We have

$$
\begin{aligned}
& {\left[\left(\chi_{1} \partial_{x}, r_{1}, v_{1} d x\right)_{\nabla, \Pi},\left(\chi_{2} \partial_{x}, r_{2}, v_{2} d x\right)_{\nabla, I}\right]_{-1,-1}} \\
& =\chi_{1} v_{2}+\chi_{2} v_{1}+\operatorname{tr}\left(-r_{1} r_{2}+\frac{1}{2}\left(r_{1} \chi_{2}^{\prime}+r_{2} \chi_{1}^{\prime}-r_{2}^{\prime} \chi_{1}-r_{1}^{\prime} \chi_{2}\right)\right. \\
& \left.\quad+\frac{1}{6}\left(\chi_{1}^{\prime \prime} \chi_{2}+\chi_{1} \chi_{2}^{\prime \prime}-\chi_{1}^{\prime} \chi_{2}^{\prime}\right)\right) .
\end{aligned}
$$

For $a \in \mathscr{A}_{\pi}={ }^{\operatorname{tr}} \mathscr{A}^{0}$ suppose that $\varepsilon(a)=\tau(x, s) \partial_{x}+\mu(s) \partial_{s}, \operatorname{ad}_{a}(\nabla)=\omega d x$. Then

$$
\begin{gathered}
{\left[a,\left(\chi \partial_{x}, r, v d x\right)_{V, \Pi}\right]_{0 .-1}=\left(\left[\varepsilon(a), \chi \partial_{x}\right], \omega \chi+[a, r]\right.} \\
\left.\operatorname{Lie}_{\varepsilon(a)}(v d x)+\operatorname{tr}\left(\frac{1}{2} r \tau^{\prime \prime}+\frac{1}{6} \chi \tau^{\prime \prime \prime}-r \omega-\frac{1}{2} \chi \omega^{\prime}\right)\right) .
\end{gathered}
$$

If $\nabla$ is a restriction on the fiber of an integrable connection $\tilde{\nabla}$, so that $a=\tilde{\nabla}(\varepsilon(a))+A$, then $\omega=-A^{\prime}$, and the formula transforms to

$$
\begin{gathered}
{\left[a,\left(\chi \partial_{x}, r, v d x\right)_{\nabla, \Pi}\right]=\left(\left[\varepsilon(a), \chi \partial_{x}\right],-A^{\prime} \chi+[a, r]\right.} \\
\left.\operatorname{Lie}_{\varepsilon(a)}(v d x)+\operatorname{tr}\left(-r A^{\prime}+\frac{1}{2}\left(r \tau^{\prime \prime}-\chi A^{\prime \prime}\right)+\frac{1}{6} \chi \tau^{\prime \prime \prime}\right)\right) .
\end{gathered}
$$




\section{A3. Local Riemann-Roch}

Let $\mathscr{A}$ be a $R$-Atiyah algebra with trace $\operatorname{tr}[\operatorname{see}(1.1 .7)]$. Consider the following $\mathcal{O}_{X^{-}}$ bilinear $\Omega$-extensions:

$-\tilde{\mathscr{A}}_{1}:=(,) \mathscr{A},(A, B)=\frac{1}{2} \operatorname{tr} A B$.

- $\tilde{\mathscr{A}}_{2}$ : this is the $\Omega$-extension of $\mathcal{O}_{X} \times \mathcal{O}_{X}$-Atiyah algebra $\operatorname{tr} \mathscr{A} \times \mathscr{T}_{X} \mathscr{A}_{\omega}$ corresponding to the bilinear form $-\frac{1}{2}\langle$,$\rangle , where \left\langle\left(f_{1}, g_{1}\right),\left(f_{2}, g_{2}\right)\right\rangle=\frac{1}{2}\left(f_{1} g_{2}+f_{2} g_{1}\right)$.

- $\tilde{\mathscr{A}}_{3}$ : $\Omega$-extension of $\mathscr{A}_{\Omega_{X / S}^{1}}$ corresponding to the bilinear form $\left(f_{1}, f_{2}\right)$ $=\frac{n}{12} f_{1} f_{2}, n=\mathrm{rk} \mathscr{A}:=\operatorname{tr} 1$. algebra.

${ }^{R R} \tilde{\mathscr{A}}:=\tilde{\mathscr{A}}_{1} \times \tilde{\mathscr{A}}_{2} \times \tilde{\mathscr{A}}_{3}$. This is an $\Omega$-extension of $\left(R \times \mathcal{O}_{X} \times \mathcal{O}_{X} \times \mathcal{O}_{X}\right)$-Atiyah

According to A1 ${ }^{R R} \tilde{\mathscr{A}}$ has the following description.

A3.1. For any pair of relative connections $\nabla_{\mathscr{A}}, \nabla_{\Omega}$ on $\mathscr{A}$ and $\Omega_{X / S}^{1}$ respectively we have the isomorphism,

$$
\begin{aligned}
& S_{\nabla_{\mathscr{A}}, \nabla_{\Omega}}: \mathscr{T}_{X / S} \oplus R \oplus(\mathcal{O} \oplus \mathcal{O}) \oplus \mathcal{O} \oplus \Omega_{X / S}^{1} \stackrel{\sim}{\rightarrow}^{R R} \mathscr{A}^{-1}, \\
& S_{V_{\mathscr{A}}, \nabla_{S 2}}(\chi, B, f, \ell, h, v)=:(\chi, B, f, \ell, h, v)_{\nabla_{\mathscr{A}}, \nabla_{\Omega}},
\end{aligned}
$$

such that

$$
\begin{aligned}
& (\chi, B, f, \ell, h, v)_{V_{\mathscr{A}}+\omega, \nabla_{\Omega}}=(\chi, \omega \chi+B, \operatorname{tr} \omega \chi \\
& \left.\quad+f, \ell, h, \frac{1}{2} \operatorname{tr} \omega^{2} \chi+\operatorname{tr} \omega B-\frac{1}{2} \operatorname{tr} \omega \cdot \ell+v\right)_{\nabla_{\mathscr{A}}, \nabla_{\Omega}} \\
& (\chi, B, f, \ell, h, v)_{\nabla_{\mathscr{S}}, \nabla_{\Omega}+\lambda} \\
& \quad=\left(\chi, B, f, \lambda \chi+\ell, \lambda \chi+h,-\frac{1}{2} \lambda f+\frac{n}{12} \lambda^{2} \chi+\frac{n}{6} \lambda h+v\right)_{\nabla_{\mathscr{A}}, V_{\Omega}} .
\end{aligned}
$$

The bracket is given by formulas [here $\bar{a}=(a, b, c, d){ }^{R R} \mathscr{A}^{0}=\left(\mathscr{A} \times_{\mathscr{T}_{X}}(\operatorname{tr} \mathscr{A}\right.$ $\left.\left.\left.\times_{\mathscr{T}_{X}} \mathscr{A}_{\Omega_{X / S}^{1}}\right) \times_{\mathscr{T}_{X}} \mathscr{A}_{\Omega_{X / S}^{1}}\right)_{\pi}\right]$ :

$$
\begin{aligned}
& {\left[\bar{a},(\chi, B, f, \ell, h, v)_{\nabla_{\mathscr{A}}, \nabla_{\Omega}}\right]_{0,-1}} \\
& \quad=\left([\varepsilon(a), \chi], \operatorname{ad}_{a}\left(\nabla_{\mathscr{A}}\right) \chi+[a, B], \operatorname{ad}_{b}\left(\operatorname{tr} \nabla_{\mathscr{A}}\right) \cdot \chi+[b, f],\right. \\
& \operatorname{ad}_{c}\left(\nabla_{\Omega}\right) \cdot \chi+[c, \ell], \operatorname{ad}_{d}\left(\nabla_{\Omega}\right) \cdot \chi+[d, h], \operatorname{tr}\left(B \cdot \operatorname{ad}_{a}\left(\nabla_{\mathscr{A}}\right)\right. \\
& \left.\quad-\quad \frac{1}{2} \operatorname{ad}_{b}\left(\operatorname{tr} \nabla_{a}\right) \cdot \ell-\frac{1}{2} \operatorname{ad}_{c}\left(\nabla_{\Omega}\right) \cdot f+\frac{n}{6} \operatorname{ad}_{d}\left(\nabla_{\Omega}\right) \cdot h+\operatorname{Lie}_{\varepsilon(a)}(v)\right)_{\nabla_{\mathscr{A}}, \nabla_{\Omega}}, \\
& {\left[\left(\chi_{1}, B_{1}, f_{1}, \ell_{1}, h_{1}, v_{1}\right)_{\nabla_{\mathscr{A}}, \nabla_{\Omega}},\left(\chi_{2}, B_{2}, f_{2}, \ell_{2}, h_{2}, v_{2}\right)_{V_{\mathscr{A},}, \nabla_{\Omega}}\right]_{-1,-1}} \\
& \quad=\chi_{1} v_{2}+v_{1} \chi_{2}-\operatorname{tr} B_{1} B_{2}+\frac{1}{2}\left(f_{1} \ell_{2}+\ell_{1} f_{2}\right)-\frac{n}{6} h_{1} h_{2} .
\end{aligned}
$$

A3.2. Let us return to A2.4. A relative connection $\nabla_{\Omega}$ on $\Omega_{X / S}^{1}$ defines the $\mathcal{O}_{X}$-parametrix $\Pi_{\nabla_{\Omega}}$ as follows (cf. 5.3): Consider the equation $\nabla_{\Omega}(f)+f^{2}=0$, where $f \in \mathscr{P}$ and $\nabla_{\Omega}$ acts along the second coordinate. This equation has a unique solution with a pole at $\Delta$; this is our parametrix $\Pi_{\nabla_{\Omega}}$.

Remark. Here is an equivalent description of $\Pi_{V_{\Omega}}$. Let $\omega(x)$ be a non-zero $\nabla_{\Omega^{2}}$-horizontal form, and $\varphi\left(x_{1}, x_{2}\right):=\int_{x_{1}}^{x_{2}} \omega \in \mathcal{O}_{\hat{A}}$ be a function such that $\varphi(x, x)=0$, 
$d_{x_{2}} \varphi\left(x_{1}, x_{2}\right)=\omega\left(x_{2}\right)$. Then $\Pi_{\nabla_{\Omega}}=\frac{\omega\left(x_{2}\right)}{\varphi\left(x_{1}, x_{2}\right)}$. If we choose a local coordinate $x$ such that $\nabla_{\Omega}(d x)=0$ (we will say that $x$ is $\nabla_{\Omega}$-flat; it is defined up to a transformation $x \mapsto a x+b, a, b \in \mathbb{C}, a \neq 0$, and $\nabla_{\Omega}$ is the same as the class of such coordinates) then $\Pi_{\nabla_{\Omega}}=\frac{d x_{2}}{x_{2}-x_{1}}$.

So, according to $\mathrm{A} 2.4,\left(\nabla_{\mathscr{A}}, \nabla_{\Omega}\right)$ determine the isomorphism

$$
\begin{gathered}
\mathscr{T}_{X / S} \oplus R \oplus \Omega_{X / S}^{1} \tilde{S}^{\operatorname{tr}} \mathscr{A}^{-1}, \\
(\chi, r, v) \mapsto(\chi, r, v)_{\nabla_{\mathscr{A}}, \nabla_{s 2}}:=(\chi, r, v)_{\nabla_{\mathscr{A}}, I_{\nabla_{\Omega}}} .
\end{gathered}
$$

A3.3. Theorem-Definition. Define the Riemann-Roch map $R R_{\mathscr{A}}:{ }^{\mathrm{tr}} \mathscr{A} \rightarrow{ }^{R R} \tilde{\mathscr{A}}$ by formulas

$$
\begin{aligned}
& R R^{0}(a)=\left(a, \operatorname{tr} a, \operatorname{Lie}_{\varepsilon(a)}, \operatorname{Lie}_{\varepsilon(a)}\right), \\
& R R^{-1}(\chi, r, v)_{\nabla_{\mathscr{S}}, \nabla_{S \Omega}}=\left(\chi, r, \operatorname{tr} r, \chi^{\prime}, \chi^{\prime}, v-\frac{1}{2} \operatorname{tr} \nabla_{\mathscr{A}}(r)+\frac{n}{6} \chi^{\prime \prime}\right)_{\nabla_{\mathscr{A}}, \nabla_{\Omega}},
\end{aligned}
$$

where $\mathrm{Lie}_{\varepsilon(a)} \in \mathscr{A}_{\Omega_{X / S}}$ is Lie derivative, and 'means derivation by $\nabla_{\Omega}$ (i.e. $\left.\mathscr{T}_{X / S} \rightarrow \mathscr{T}_{X / S} \otimes \Omega_{X / S}^{1}=\mathcal{O}_{X}\right)$. This is correctly defined morphism of $\pi$-algebras.

The proof is direct calculation (the formula for $R R^{-1}$ may be found from the demand that $R R$ commutes with [ $]_{-1,-1}$ ).

Integrating A 3.3 along the fibers of $\pi$ we get the isomorphism $\pi(R R): \pi\left({ }^{\mathrm{tr}} \mathscr{A}\right)$ $\cong \pi\left(\tilde{\mathscr{A}}_{1}\right)+\pi\left(\tilde{\mathscr{A}}_{2}\right)+\pi\left(\tilde{\mathscr{A}}_{3}\right)$ of $\mathcal{O}_{X}$-Atiyah algebras.

A4.

Remark. Let $\nabla_{\mathscr{A}}$ be a $\mathrm{C}^{\infty}$-connection on $\mathscr{A}$ (see 5.1), and $\nabla_{\Omega}$ be the one of $\Omega_{X / S}^{1}$. According to 1.5 they define the $\mathrm{C}^{\infty}$-connection ${ }^{R R} \tilde{V}$ on ${ }^{R R} \tilde{\mathscr{A}}$. Using the method of 5.4 one can also construct a $C^{\infty}$-connection ${ }^{\operatorname{tr}} \tilde{\nabla}$ on ${ }^{\operatorname{tr}} \mathscr{A}$, and $R R$ maps ${ }^{\operatorname{tr}} \tilde{\nabla}$ to ${ }^{R R} \tilde{V}$.

The formulations of Sect. 6 suggest that this result may be generalized. Namely, there should exist some kind of functoriality of $R R$ with respect to $\mathrm{C}^{\infty}$-maps of Atiyah algebras.

\section{A5. Compatibility with Deligne's Riemann-Roch}

Let $E$ be a rank $n$ vector bundle and $L_{1}, L_{2}$ be invertible sheaves on $X$. Put $\omega:=\Omega_{X / S}^{1}$. In [11] Deligne defined the invertible sheaves $\mathrm{IC}_{2}(E),\left\langle L_{1}, L_{2}\right\rangle$ on $S$ and constructed the Riemann-Roch isomorphism

$$
R R_{E}: \operatorname{det} R \pi_{*} E^{\otimes 12} \tilde{\rightarrow}\langle\operatorname{det} E, \operatorname{det} E\rangle^{\otimes 6} \cdot \mathrm{IC}_{2}(E) \cdot\langle\operatorname{det} E, \omega\rangle^{\otimes-6} \cdot\langle\omega, \omega\rangle^{\otimes n} .
$$

In this section we will see that the $R R_{\mathscr{A}_{E}}$ isomorphism of A3.3, integrated along the fibers of $E$, is $R R_{E}$ on the level of Atiyah algebras: $\pi\left(R R_{\mathscr{A} E}\right)=\mathscr{A}_{R R_{E}}$ or, equivalently, that $\pi\left(R R_{\mathscr{A}_{F}}\right)$ is the differential equation for $R R_{E}$. We will start with the identification of Deligne's sheaves.

A 5.1. We will use the following variant of Deligne's construction. Let $\hat{S \mathrm{~L}}_{n}$ be a universal extension of $\mathrm{SL}_{n}\left(\mathcal{O}_{X}\right)$ by $\mathscr{K}_{2}$ if $n=2$ put $\left.\mathrm{SL}_{2}=\left.\hat{\mathrm{SL}}_{3}\right|_{\mathrm{SL}_{2}}\right)$. The standard $\mathrm{GL}_{1}$ 
$=\left\{\left(* 1_{1}\right)\right\} \subset \mathrm{GL}_{n}$ acts on $\mathrm{SL}_{n}$ by conjugation, hence on $\hat{\mathrm{SL}}_{n}$; put ${ }^{{ }^{c}} \hat{\mathrm{GL}}_{n}=\mathrm{GL}_{1} \bowtie$ $\widehat{S L}_{n}$. We get a central extension

$$
0 \rightarrow \mathscr{K}_{2} \rightarrow{ }^{\mathrm{c}_{2}} \hat{\mathrm{GL}}_{n} \rightarrow \mathrm{GL}_{n} \rightarrow 1 .
$$

This is just the extension corresponding to the universal Chern class $\mathrm{c}_{2} \in H^{2}\left(\mathrm{GL}_{n}, \mathscr{K}_{2}\right),[31]$. Let us denote by $s: \mathrm{GL}_{1} \rightarrow \hat{\mathrm{GL}}_{n}$ the canonical section. Now let $\mathrm{GL}_{1} \widehat{\times} \mathrm{GL}_{1}$ be the central extension,

$$
0 \rightarrow \mathscr{K}_{2} \rightarrow \mathrm{GL}_{1} \widehat{\times} \mathrm{GL}_{1} \rightarrow \mathrm{GL}_{1} \times \mathrm{GL}_{1} \rightarrow 1,
$$

defined by 2-cocycle $\left\{p r_{1}, p r_{2}\right\}$, i.e. $\mathrm{GL}_{1} \widehat{\times} \mathrm{GL}_{1}=\mathrm{GL}_{1} \times \mathrm{GL}_{1} \times \mathscr{K}_{2}$ with multiplication $(f, g ; r)\left(f^{\prime}, g^{\prime} ; r^{\prime}\right)=\left(f f^{\prime}, g g^{\prime}, r \cdot r^{\prime} \cdot\left\{f^{\prime}, g\right\}\right)$. These extensions have the following properties:

(i) For $\alpha \in \mathrm{GL}_{1}$ and $\beta=\left(\begin{array}{cc}\beta_{1} & * \\ 0 & \beta_{2}\end{array}\right), \beta_{1} \in \mathrm{GL}_{1}, \beta_{2} \in \mathrm{GL}_{n-1}$, one has $\operatorname{ad}_{\beta}(s(\alpha))$ $=\left\{\operatorname{det} \beta_{2}, \alpha\right\}$.

(ii) Define the section $s_{n}$ of ${ }^{{ }^{2}} \hat{\mathrm{GL}_{n}}$ on the diagonal matrices $\mathrm{T}_{n} \subset \mathrm{GL}_{n}$ by the formula $s_{n}\left(\operatorname{diag}\left(a_{1}, \ldots, a_{n}\right)\right)=s\left(a_{1}\right) \cdot \operatorname{ad}_{\sigma_{2}}\left(s\left(a_{2}\right)\right) \cdot \ldots \cdot \operatorname{ad}_{\sigma_{n}}\left(s\left(a_{n}\right)\right)$, where $\sigma_{i}$ is a transposition of coordinates such that $\left(\sigma_{i}\right)_{\alpha 1}=\delta_{i \alpha}$. Then $s_{n}\left(\operatorname{diag}\left(a_{i}\right)\right) \cdot s_{n}\left(\operatorname{diag}\left(b_{i}\right)\right)$ $=s_{n}\left(\operatorname{diag}\left(a_{i} b_{i}\right)\right) \cdot \prod_{i<j}\left\{a_{i}, b_{j}\right\}$.

(iii) One has a canonical group-theoretic section of ${ }^{\mathrm{c}_{2}} \hat{\mathrm{GL}}_{n}$ on $\mathrm{U}_{n}=\left\{\left(\begin{array}{lll}1 & & * \\ 0 & & 1\end{array}\right)\right\}$.

(iv) For $\quad n=n_{1}+n_{2} \quad$ consider $\quad \mathrm{P}_{n_{1} n_{2}}=\left\{\left(\begin{array}{cc}\mathrm{GL}_{n_{1}} & * \\ 0 & \mathrm{GL}_{n_{2}}\end{array}\right)\right\} \subset \mathrm{GL}_{n} ; \quad$ let $p_{i}: \mathrm{P}_{n_{1} n_{2}} \rightarrow \mathrm{GL}_{n_{2}}$ be the projections. Then one has a canonical isomorphism of $\hat{\mathrm{GL}}_{n}$ restricted to $\mathrm{P}_{n_{1} n_{2}}$ with the sum of extensions $p_{1}^{*}\left(\hat{\mathrm{GL}} \mathrm{n}_{n_{1}}\right) \cdot p_{2}^{*}\left(\hat{\mathrm{GL}}_{n_{2}}\right) \cdot\left(\operatorname{det} p_{1}\right.$ $\left.\times \operatorname{det} p_{2}\right)^{*}\left(\mathrm{GL}_{1} \widehat{\times} \mathrm{GL}_{1}\right)$. Namely, it is unique isomorphism that maps $s_{n}\left(\operatorname{diag}\left(a_{i}\right)\right)$ to

$$
\begin{aligned}
& s_{n_{1}}\left(\operatorname{diag}\left(a_{1}, \ldots, a_{n_{1}}\right)\right) \cdot s_{n_{2}}\left(\operatorname{diag}\left(a_{n_{1}+1}, \ldots, a_{n}\right)\right) \\
& \cdot\left(a_{1} \cdot \ldots \cdot a_{n_{1}}, a_{n_{1}+1} \cdot \ldots \cdot a_{n}, 1\right) .
\end{aligned}
$$

Now our vector bundle $E$ is the same as $\mathrm{GL}_{n}$-torsor $\mathscr{E}=\operatorname{Isom}\left(\mathcal{O}^{n}, E\right)$. Consider the presheaf on $S$ whose sections over $U \subset S$ are isomorphism classes of $\hat{\mathrm{GL}}_{n}$-structures on $\pi^{-1}(U)$ (here $\hat{\mathrm{GL}}_{n}$-structure means a $\hat{\mathrm{GL}}_{n}$-torsor $\widehat{\mathscr{E}}$ together with an isomorphism $\mathscr{E} \cong \hat{\mathscr{E}} / \mathscr{K}_{2}$ ). Since fibers of $\pi$ are one-dimensional, the corresponding sheaf is $R^{1} \pi_{*} \mathscr{K}_{2}$-torsor. The norm map $R^{1} \pi_{*} \mathscr{K}_{2} \rightarrow \mathcal{O}_{S}^{*}$ applied to this torsor gives us $\mathcal{O}_{S}^{*}$-torsor which is just Deligne's sheaf $\operatorname{IC}_{2}(E)$. The sheaf $\left\langle L_{1}, L_{2}\right\rangle$ may be defined in the same way using $\mathrm{GL}_{1} \widehat{\times} \mathrm{GL}_{1}$ and $\mathcal{O}^{*} \times \mathcal{O}^{*}$-torsor $\mathscr{L}_{1} \times \mathscr{L}_{2}$. Note that (iv) above associates with any short exact sequence $0 \rightarrow E_{1} \rightarrow E$ $\rightarrow E_{2} \rightarrow 0$ the isomorphism $\mathrm{IC}_{2}(E)=\mathrm{IC}_{2}\left(E_{1}\right) \mathrm{IC}_{2}\left(E_{2}\right)\left\langle\operatorname{det} E_{1}, \operatorname{det} E_{2}\right\rangle$.

Remark. Deligne's definition is somewhat different but may readily reduce to the one above.

A 5.2. Now let $(,)_{\mathrm{c}_{2}}$ be the invariant form on $\mathrm{gl}_{n}:=\mathrm{Lie}_{\mathrm{GL}}$ given by formula $(X, Y)_{\mathrm{c}_{2}}=\operatorname{tr} X \operatorname{tr} Y-\operatorname{tr} X Y$. Let $(,)_{\mathrm{c}_{1} \cdot \mathrm{c}_{1}}$ be the form $\left(\left(f_{1}, g_{1}\right),\left(f_{2}, g_{2}\right)\right)_{\mathrm{c}_{1} \cdot \mathrm{c}_{1}}$ $=\frac{1}{2}\left(f_{1} g_{2}+g_{1} f_{2}\right)$ on $\mathcal{O}_{X} \times \mathcal{O}_{X}$. Consider the corresponding $\mathcal{O}_{X}$-bilinear $\Omega$-extensions ${ }^{c_{2}} \mathscr{A}_{E}$ of $\mathscr{A}_{E}$ and ${ }^{\mathrm{c}_{1} \cdot{ }^{\mathrm{c}_{1}}} \mathscr{A}_{L_{1} L_{2}}$ of $\mathscr{A}_{L_{1}} \times \mathscr{A}_{L_{2}}$. 
Lemma-Construction. One has canonical isomorphisms

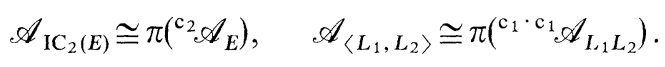

Proof. We will treat the case $\mathrm{IC}_{2}$ only; the $c_{1} \cdot c_{1}$ case is parallel. Our $\mathcal{O}_{S}$-Atiyah algebras are locally trivial, hence they are completely determined by the corresponding $\Omega_{S}^{1}{ }^{c l}$-torsors of integrable connections $\mathscr{I} \mathscr{C}(A):=\operatorname{Isom}\left(\mathscr{A}_{\mathcal{O}_{S}}, \mathscr{A}\right)$. So it suffices to identify $\mathscr{I} \mathscr{C}\left(\mathscr{A}_{\mathrm{IC}_{2}(E)}\right)$ with $\mathscr{I} \mathscr{C}\left(\pi^{\mathrm{c}_{2}} \mathscr{A}_{E}\right)$. Since $\mathscr{I} \mathscr{C}\left(\mathscr{A}_{\mathrm{IC}_{2}(E)}\right)=d \log \mathrm{IC}_{2}(E)$ [where $\mathrm{IC}_{2}(E)$ is considered as a $\mathcal{O}_{S}^{*}$-torsor] this will be done if we define a $d$ logmorphism $\varphi: \mathrm{IC}_{2}(E) \rightarrow \mathscr{I} \mathscr{C}\left(\pi^{\mathrm{c}_{2}}\left(\mathscr{A}_{E}\right)\right)$. Now recall that in 1.3.3 the canonical map $\psi: P_{E}:=\left\{\right.$ isomorphism classes of $\mathscr{H}^{1}$-extensions of $\left.{ }^{c_{2}} \mathscr{A}_{E}\right\} \rightarrow \mathscr{I} \mathscr{C}\left(\pi^{\mathrm{c}_{2}} \mathscr{A}_{E}\right)$ was constructed; this map is equivariant with respect to $R^{1} \pi_{*}\left(\widetilde{\Omega}_{S}^{c l}\right)$-twist. Our $\varphi$ will be a composition $\operatorname{IC}_{2}(E) \stackrel{\chi}{\longrightarrow} P_{E} \stackrel{\psi}{\longrightarrow} \mathscr{I} \mathscr{C}\left(\pi^{\mathrm{c}_{2}} \mathscr{A}_{E}\right)$. Note that $\mathrm{IC}_{2}(E)$ has natural $R^{1} \pi_{*} \mathscr{K}_{2}$-action, $P_{E}$ has the one of $R^{1} \pi_{*} \widetilde{\Omega}_{S}^{c l}$, and $\chi$ will be equivariant with respect to $\alpha: R^{1} \pi_{*} \mathscr{K}_{2} \rightarrow R^{1} \pi_{*} \widetilde{\Omega}_{S}^{c l}$, where $\alpha$ comes from the same noted map $\alpha: \mathscr{K}_{2} \stackrel{d \log }{\longrightarrow} \Omega_{X}^{2 c l}$ $\rightarrow \widetilde{\Omega}_{S}^{c l}$, the last arrow is "restriction to fiber." This would imply that $\varphi$ is $d \log$ equivariant.

Recall that ${ }^{\mathrm{c}_{2}} \mathscr{A}_{E}$ was constructed as follows. We have the algebra ${ }^{\mathrm{c}_{2}} \mathscr{A}_{\mathcal{O}^{n}}$ with $\mathrm{GL}_{n}\left(\mathcal{O}_{X}\right)$-action, and ${ }^{c_{2}} \mathscr{A}_{E}$ is $\mathscr{E}$-twist of it $\left(\mathcal{O}=\mathcal{O}_{X}\right)$. Now we will construct the $\mathscr{H}^{1}$-extension ${ }^{\mathrm{c}_{2}} \hat{\mathscr{A}}$ of ${ }^{\mathrm{c}_{2}} \mathscr{A}_{\mathcal{O}^{n}}$ together with natural $\mathrm{GL}_{n}\left(\mathcal{O}_{X}\right)$-action that reduces to

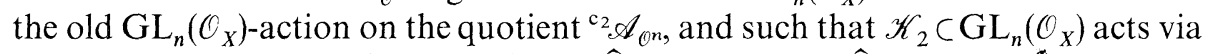
$\alpha$ (see 1.3.3). The map $\chi$ just transforms $\widehat{\mathscr{E}} \in \mathrm{IC}_{2}(E)$ to an $\widehat{\mathscr{E}}$-twist of ${ }^{\mathrm{c}_{2}} \mathscr{\mathscr { A }}$; clearly it will have the desired properties.

Construction of ${ }^{\mathrm{c}_{2}} \hat{\mathscr{A}}$ : we have to define ${ }^{{ }^{\mathrm{c}} \mathscr{A}} \mathscr{A}^{0}$ only. Put ${ }^{\mathrm{c}_{2}} \mathscr{A}^{0}$ $=\mathscr{T}_{\pi} \oplus \operatorname{Mat}_{n}\left(\mathcal{O}_{X}\right) \oplus \mathscr{H}^{1}$. Bracket:

$$
\begin{aligned}
& {\left[\left(\chi_{1}, r_{1}, v_{1}\right),\left(\chi_{2}, r_{2}, v_{2}\right)\right]=\left(\left[\chi_{1}, \chi_{2}\right], \chi_{1}\left(r_{2}\right)-\chi_{2}\left(r_{1}\right)\right.} \\
& \left.\quad+\left[r_{1}, r_{2}\right], \chi_{1}\left(v_{2}\right)-\chi_{2}\left(v_{1}\right)+2\left(r_{2}, d r_{1}\right)\right),
\end{aligned}
$$

where $\left(r_{2}, d r_{1}\right)$ is the scalar product with respect to the $\mathrm{c}_{2}$-form. One verifies that $[$,$] is a Lie algebra bracket; in fact, { }^{c_{2}} \mathscr{A}^{0}$ is a semi-direct product of $\mathscr{T}_{\pi}$ and the Kac-Moody part $\operatorname{Mat}_{n}\left(\mathcal{O}_{X}\right) \oplus \mathscr{H}^{1}$.

Construction of $\mathrm{GL}_{n}$-action: it "integrates" the adjoint action of the KacMoody part. So let $\hat{G}$ be the group of automorphisms of sheaf ${ }^{c_{2}} \hat{\mathscr{A}}^{0}$ that preserve the flag $\mathscr{H}^{1} \subset \mathscr{H}^{1} \oplus \mathrm{Mat}_{n}\left(\mathcal{O}_{X}\right)$ and induce identity maps on $\mathscr{H}^{1}, \mathscr{T}_{\pi}$ $={ }^{{ }^{2}} \hat{\mathscr{A}}^{0} /\left(\mathscr{H}^{1} \oplus \operatorname{Mat}_{n}\left(\mathscr{O}_{X}\right)\right)$; let $A=\operatorname{Hom}\left(\mathscr{T}_{\pi}, \mathscr{H}^{1}\right) \subset \widehat{G}$ be the subgroup of ones that induce identity maps on $\mathscr{H}^{1} \oplus \operatorname{Mat}_{n}\left(\mathcal{O}_{X}\right)$ and ${ }^{\mathrm{c}_{2}} \hat{\mathscr{A}}^{0} / \mathscr{H}^{1}$; put $G=\hat{G} / A$. Then $\hat{G}$ is a central extension of $G$ by $A$. Define the map $\Phi: \mathrm{GL}_{n}\left(\mathcal{O}_{X}\right) \rightarrow \hat{G}$ by the formula

$$
\Phi(g)(\chi, r, v)=\left(\chi, g \chi g^{-1}-\chi(g) g^{-1}, v+2\left(d g g^{-1}, r\right)\right) .
$$

One verifies that both $\Phi \bmod A: \mathrm{GL}_{n}\left(\mathcal{O}_{X}\right) \rightarrow G$ and $\left.\Phi\right|_{\mathrm{GL}_{1}}: \mathrm{GL}_{1} \rightarrow \widehat{G}$ are group morphisms. Hence the universality property of $\hat{G L}$ implies that there exists a unique group morphism $\hat{\Phi}: \hat{\mathrm{GL}}_{n} \rightarrow \widehat{G}$ such that $\hat{\Phi} \bmod A=\Phi, \hat{\Phi} \circ S=\left.\Phi\right|_{\mathrm{GL}_{1}}$. So we get a desired $\hat{G L}_{n}$-action on ${ }^{c_{2}} \hat{\mathscr{A}}$.

A 5.3. By (2.3.1), A 3.3 and A 5.2 we have a canonical isomorphism between Atiyah algebras of invertible sheaves

$$
\begin{gathered}
\left(\operatorname{det} R \pi_{*} E\right)^{\otimes 12} \text { and }\langle\operatorname{det} E, \operatorname{det} E\rangle^{\otimes 6} \cdot \mathrm{IC}_{2}(E)^{\otimes-12} \\
\cdot\langle\operatorname{det} E, \omega\rangle^{\otimes-6} \cdot\langle\omega, \omega\rangle^{\otimes n}
\end{gathered}
$$


or, equivalently, the integrable connection on their quotient. Of course we have

Lemma. This isomorphism comes from Deligne's Riemann-Roch isomorphism, [11]:

$$
R R_{E}: \operatorname{det} R \pi_{*} E^{\otimes 12} \cong\langle\operatorname{det} E, \operatorname{det} E\rangle^{\otimes 6} \cdot \mathrm{IC}_{2}(E)^{\otimes-12} \cdot\langle\operatorname{det} E, \omega\rangle^{\otimes-6} \cdot\langle\omega, \omega\rangle^{\otimes n}
$$

Sketch of the Proof. Deligne's isomorphism may be characterised by the following properties (see [11] for details):

(i) compatibility with base change;

(ii) compatibility with filtrations on $E$ 's;

(iii) if $\operatorname{rk} E=1, a \in X$ is $S$-point, then we have canonical identifications

$$
\begin{gathered}
\frac{\operatorname{det} R \pi_{*} E(a)}{\operatorname{det} R \pi_{*} E}=E_{a} \otimes \mathscr{T}_{a}, \mathrm{IC}_{2}(E)=\mathrm{IC}_{2}(E(a))=\mathcal{O}_{S}, \\
\langle E(a), E(a)\rangle=\langle E, E\rangle E_{a}^{\otimes 2} \mathscr{T}_{a},\langle E(a), \omega\rangle=\langle E, \omega\rangle \cdot \mathscr{T}_{a}^{-1} .
\end{gathered}
$$

The $R R$-isomorphisms should be compatible with these identifications.

(iv) Consider $R R_{\omega}:\left(\operatorname{det} R \pi_{*} \omega\right)^{\otimes 12} \stackrel{\sim}{\rightarrow}\langle\omega, \omega\rangle$,

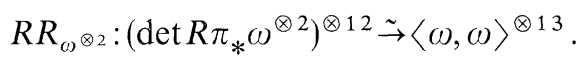

On the other hand we have the (suitably normalised) Mumford isomorphism,

Then

$$
\mu: \operatorname{det} R \pi_{*} \omega^{\otimes 2} \stackrel{\sim}{\rightarrow}\left(\operatorname{det} R \pi_{*} \omega\right)^{\otimes 13} .
$$

$$
R R_{\omega \otimes 2} \cdot\left(R R_{\omega}^{\otimes 13}\right)^{-1}=\mu^{\otimes 12} .
$$

To prove the lemma we have to verify these properties for our isomorphism on the Atiyah algebra level. (i) is obvious. (ii): let $E_{i} \subset E$ be a filtration; then ${ }^{\operatorname{tr}} \mathscr{A}_{E}$ contains a $\pi$-subalgebra ${ }^{\operatorname{tr}} \mathscr{A}_{\left(e, E_{i}\right)}$ with ${ }^{\operatorname{tr}} \mathscr{A}_{\left(E, E_{1}\right)}^{0}=$ infinitesimal automorphisms of $\left(E, E_{i}, X, S\right)$. Same for ${ }^{R R} \mathscr{A}_{E}$. The $R R$-isomorphism preserves these subalgebras. We have

$$
\pi\left({ }^{\mathrm{tr}} \mathscr{A}_{E}\right)=\pi\left({ }^{\mathrm{tr}} \mathscr{A}_{\left(E, E_{l}\right)}\right)=\sum_{i} \pi\left({ }^{\mathrm{tr}} \mathscr{A}_{E_{\imath} / E_{\imath-1}}\right)
$$

the same for ${ }^{R R} \mathscr{A}_{E}$; this proves (ii). As for (iii), you should use instead of $\mathscr{A}_{E}, \mathscr{A}_{E(\text { a) }}$ their common subalgebra $\mathscr{A}_{E, a}(2.3 .2),(6.2 .4)$ that consists of elements whose symbols preserve $a$ and verify the compatibilities in (2.3.2). As for (iv), see 3.1.2.

Acknowledgements. We would like to express our deep gratitude to A. Belavin, V. Drinfeld, B. Feigin, V. Knizhnik, Yu. Manin, and A. Radul for valuable discussions. We are also very grateful to L. Titova for her indispensable help in preparation of the manuscript.

Our friend, the most brilliant young physicist Vadik Knizhnik died on December 25 th, 1987. With pain and sorrow we dedicate this paper to his memory.

\section{References}

1. Alessandrini, V., Amati, D.: Properties of dual multiloop amplitudes. Nuovo Cim. 4A, N4, 793-844 (1971)

2. Beilinson, A.A.: Residues and adéles. Funct. Anal. Appl. 14, N1, $44-45$ (1980) (in Russian)

3. Beilinson, A., Bernstein, J.: Localisation de (5)-modules. C.R. Acad. Sci. (Paris) Ser. I, t. 292, 15-18 (1981)

4. Beilinson, A.A., Manin, Yu.I., Schechtman, V.V.: Sheaves of Virasoro and Neveu-Schwartz algebras. Lecture Notes in Mathematics, Vol. 1289, pp. 52-66. Berlin, Heidelberg, New York: Springer 1987 
5. Belavin, A.A., Knizhnik, V.G.: Algebraic geometry and the geometry of quantum strings. Phys. Lett. B 168, 201-206 (1986)

6. Belavin, A.A., Polyakov, A.M., Zamolodchikov, A.B.: Infinite conformal symmetries in twodimensional quantum field theory. Nucl. Phys. B 241, 333-380 (1984)

7. Bismut, J., Gillet, H., Soule, C.: Analytic torsion and holomorphic determination bundles, I, II, III. Commun. Math. Phys. 115, 49-78, 79--126, 301-351 (1988)

8. Bershadsky, M., Radul, A.: Conformal field theories with additional $Z_{N}$ symmetry. Int. J. Mod. Phys. A 2, N1, 165-178 (1987)

9. Bost, J.B.: Fibrés determinants, determinants regularisés et mesures sur les espaces de modules des courbes complexes. Sem. Bourbaki, exp. 676 (Fevr. 1987)

10. Date, E., Jimbo, M., Kashiwara, M., Miwa, T.: Transformation groups for solution equations. In: Proc. of RIMS Symposium. Jimbo, M., Miwa, T. (eds.), pp. 34-120. Singapore: World Scientific 1983

11. Deligne, P.: Le determinant de la cohomologie. In: Current trends in arithmetical algebraic geometry. Contemp. Math. 67, 93-177 (1987)

12. Feigin, B.L., Fuchs, D.B.: Representations of the Virasoro algebra. In: Seminar on supermanifolds 5. Leites, D. (ed.), Reports of Dept. Math. Stockholm Univ., N25 (1986)

13. Knizhnik, V.G.: Analytic fields on Riemann surfaces. II. Commun. Math. Phys. 112, 567-590 (1987)

14. Knudsen, F.F., Mumford, D.: The projectivity of the moduli space of stable curves. I. Preliminaries on "det" and "div". Math. Scand. 39, 19-55 (1976)

15. Kontsevich, M.L.: Virasoro algebra and Teichmüller spaces. Funct. Anal. Appl. 21, N2, 78-79 (1987) (in Russian)

16. Manin, Yu.I.: Critical dimensions of string theories and dualizing sheaf of the moduli space of (super) curves. Funct. Anal. Appl. 20, N3, 88-89 (1986) (in Russian)

17. Mumford, D.: Stability of projective varieties. Enseign. Math. 23, 39-110 (1977)

18. Quillen, D.: Determinants of Cauchy-Riemann operators over a Riemann surface. Funct. Anal. Appl. 19, No. 1, 31-34 (1985) (in Russian)

19. Segal, G., Wilson, G.: Loop groups and equations of KdV type. Publ. Math. IHES 1-64, 63 (1985)

20. Tate, J.: Residues of differentials on curves. Ann. Sci. E.N.S., 4 Ser., t. 1, 149-159 (1968)

21. Tjurin, A.N.: On periods of quadratic differentials. Russ. Math. Surv. 33, N6, 149-195 (1978)

22. Tsuchiya, A., Kanie, Y.: Fock space representations of the Virasoro algebra. Publ. RIMS 22, N2, 259-327 (1986)

23. Zamolodchikov, A1.B.: Conformal scalar field on the hyperelliptic curve and critical AshkinFeller multipoint correlation functions. Nucl. Phys. B 285, 481-503 (1987)

24. Toledo, D., Tong, Y.-L.: Duality and intersection theory in complex manifolds. I. Math. Ann. 237, 41-77 (1978)

25. Alvarez-Gaumé, L., Gomez, C., Reina, C.: New methods in string theory, preprint CERN (1987)

26. Arbarello, E., De Concini, C., Kac, V., Procesi, C.: In preparation

27. Eguchi, T., Ooguri, H.: Conformal and current algebras on a general Riemann surface. Nucl. Phys. B 282, 308 (1987)

28. Kawamoto, N., Namikawa, Yu., Tsuchiya, A., Yamada, Y.: Geometric realisation of conformal field theory on Riemann surfaces. Preprint, Nagoya university (1987)

29. Witten, E.: Quantum field theory, Grassmannians, and algebraic curves. Commun. Math. Phys. 113, 529-600 (1988)

30. Gabber, O.: The integrability of characteristic variety. Am. J. Math. 103, N3, 445 (1981)

31. Schechtman, V.V.: Riemann-Roch theorem and Atiyah-Hirzebruch spectral sequence. Usp. Mat. Nauk (= Russ. Math. Surv.), 35, N6, 179-180 (1980) (in Russian)

Communicated by A. Jaffe

Received February 12, 1988 
